(2) norden 



\section{Analysis, occurrence, and toxicity of ß-methylaminoalanine (BMAA)}

A risk for the consumer? 
Analysis, occurrence, and toxicity of ß-methylaminoalanine (BMAA)

A risk for the consumer?

TemaNord 2007:561

(C) Nordic Council of Ministers, Copenhagen 2007

ISBN 978-92-893-1541-8

Print: Ekspressen Tryk \& Kopicenter

Copies: 400

Printed on environmentally friendly paper

This publication can be ordered on www.norden.org/order. Other Nordic publications are available at www.norden.org/publications

Printed in Denmark

Nordic Council of Ministers

Store Strandstræde 18

DK-1255 Copenhagen K

Phone (+45) 33960200

Fax (+45) 33960202

www.norden.org
Nordic Council

Store Strandstræde 18

DK-1255 Copenhagen K

Phone (+45) 33960400

Fax $(+45) 33111870$

\section{Nordic co-operation}

Nordic cooperation is one of the world's most extensive forms of regional collaboration, involving Denmark, Finland, Iceland, Norway, Sweden, and three autonomous areas: the Faroe Islands, Greenland, and Åland.

Nordic cooperation has firm traditions in politics, the economy, and culture. It plays an important rol in European and international collaboration, and aims at creating a strong Nordic community in a strong Europe.

Nordic cooperation seeks to safeguard Nordic and regional interests and principles in the global community. Common Nordic values help the region solidify its position as one of the world's most innovative and competitive. 


\section{Content}

Preface

Summary in English

2. A historical perspective on the link between BMAA exposure and degenerative neurological disease.

2.1 Conclusions on cycad and BMAA exposure and degenerative neurological disease

3. Case reports on animals, toxicological studies on cycad materials and preparations.... 31

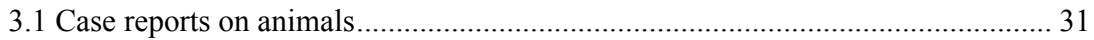

3.2 Studies on primates fed preparations from Cycas circinalis ................................ 33

3.3 Studies on rodents fed preparations from Cycas circinalis ................................... 36

3.4 Rodent studies on other cycad species than C. circinalis.................................... 39

3.5 Conclusions on case reports and toxicological studies performed with cycad preparations. ......................................................... 40

4. BMAA as a natural toxicant in cycads and studies on BMAA toxicity ........................ 41

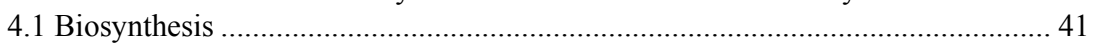

4.2 Effects in plants - a possible mechanism of action ............................................ 42

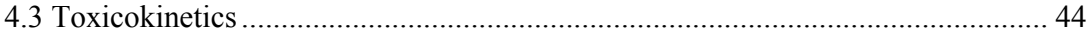

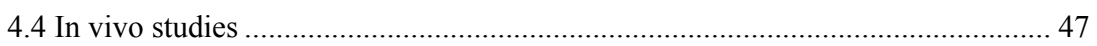

4.5 Studies on infusion of BMAA directly into brain or brain ventricles ................... 52

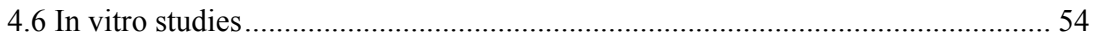

4.7 Conclusions on BMAA as a natural toxicant in cycads and

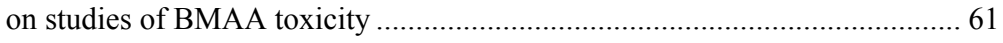

5. Identity and physical and chemical properties of BMAA, and its chemical analysis.... 63

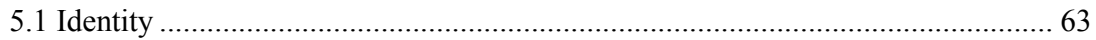

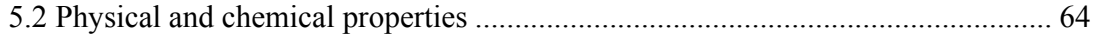

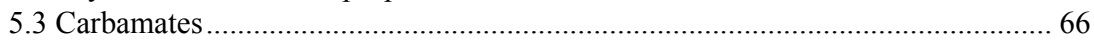

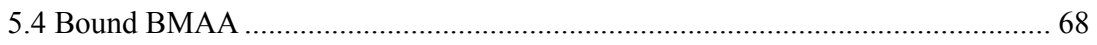

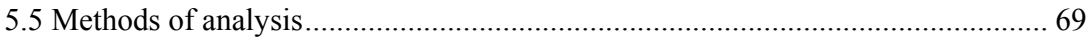

5.6 Development of an LC-MS/MS method and analysis of BMAA

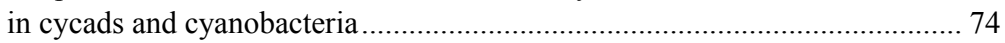

5.7 Analysis of cyanobacteria with HPLC and the ninhydrin reaction....................... 76

5.8 Conclusion on physical and chemical properties of BMAA and

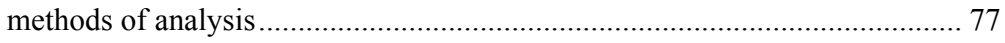

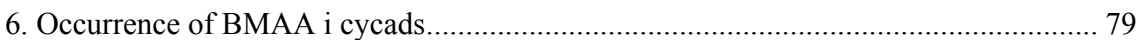

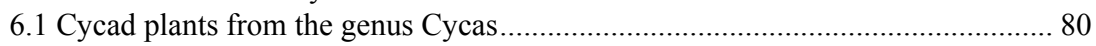

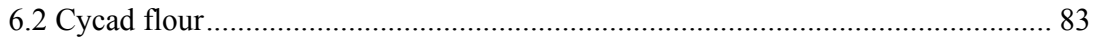

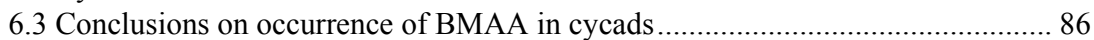


7. The theory of biomagnification and identification of BMAA in human tissues.............87

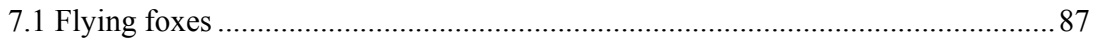

7.2 Brain material and other tissues from control individuals and patients with degenerative neurological disease............................................................91

7.3 Conclusion on the theory of biomagnification of BMAA and identification of BMAA in human tissues..............................................................................93

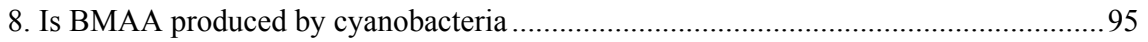

Conclusion on possible production of BMAA by cyanobacteria ................................96

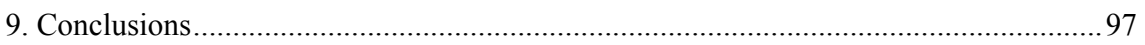

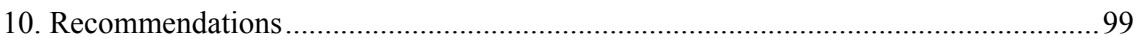

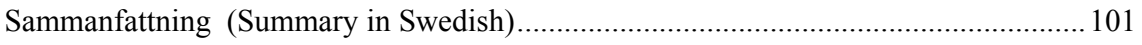

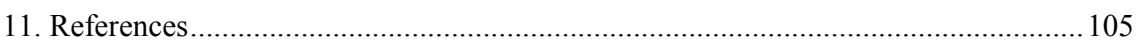

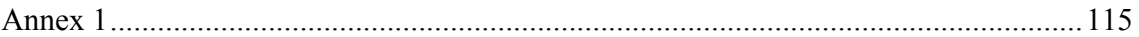

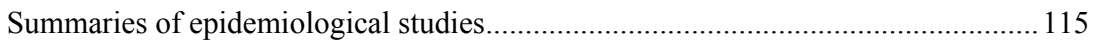

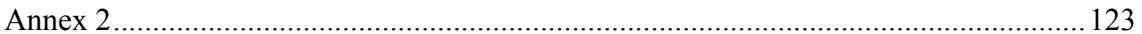

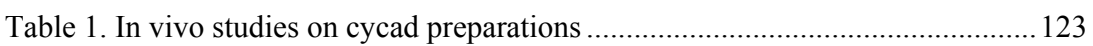

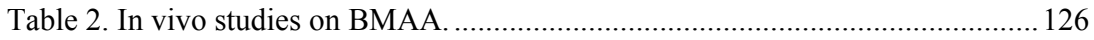




\section{Preface}

Assessment of health risks connected with exposure to naturally occurring toxicants in foodstuffs has become an important area for NNT in the recent years. A series of Nordic reports based on the work performed by the Nordic project group on inherent natural toxicants in food plants and mushrooms has been published:

- Gry, J. and Pilegaard, K. (1991) Hydrazines in the Cultivated Mushroom (Agaricus bisporus). Vår Föda 43; Supplement 1.

- Uggla, A. and Busk, L. (1992) Ethyl carbamate (urethane) in alcoholic beverages and foodstuffs - A Nordic View. Nordiske Seminar- og Arbejdsrapporter 1992:570.

- Størmer, F.C., Reistad, R. and Alexander, J. (1993) Adverse health effects of glycyrrhizic acid in licorice. A risk assessment. Nordiske Seminar- og Arbejdsrapporter 1993:526.

- Andersson, C., Slanina, P. And Koponen, A. (1995) Hydrazones in the false morel. TemaNord 1995:561.

- Søborg, I., Andersson, C. and Gry, J. (1996) Furocoumarins in Plant Food - exposure, biological properties, risk assessment and recommendations. TemaNord 1996:600.

- Gry, J. and Andersson, H.C. (1998) Nordic seminar on phenylhydrazines in the Cultivated Mushroom (Agaricus bisporus). TemaNord 1998:539.

- Andersson, H.C. (1999) Glycoalkaloids in tomatoes, eggplants, pepper and two Solanum species growing wild in the Nordic countries. TemaNord 1999:599.

- Andersson, H.C. (2003) Calystegine alkaloids in Solanaceous plants. TemaNord: 2002:513.

- Andersson, H.C., Wennström, P. and Gry, J. (2003) Nicotine alkaloids in Solanaceous food plants. TemaNord 2003:531.

- Andersson, H.C. and Gry, J. (2004) Phenylhydrazines in the Cultivated Mushroom (Agaricus bisporus). TemaNord 2004:558, $123 \mathrm{pp}$.

- Gry, J., Søborg, I. and Andersson, H.C. (2006) Cucurbitacins in plant food. TemaNord 2006:556.

Our understanding of the toxicity of the amino acid $\beta$-methylamino-Lalanine (BMAA) is intimately linked to studies on the ethiology of a progressive neurodegenerative disease among the local Chamorro people on the American island Guam in the Western Pacific. In the aftermath of World War II, U. S. Army physicians assigned to Guam, found the local 
Chamorro people to be afflicted with a progressive neurodegenerative disease described as amyotrophic lateral sclerosis (ALS) or an ALS-like condition appearing hand-in-hand with an unusual form of Parkinson dementia (PD). The incidence of amyotrophic lateral sclerosis - Parkinson dementia complex (ALS-PDC) was estimated to be 50 to 100 times higher than elsewhere in the world.

Epidemiologist early linked the traditional Chamorro diet to the occurrence of the degenerative neurological diseases. The Chamorro people harvest the seeds of the local cycad trees to prepare a flour used for baking and food production. The flour and the cycad seeds were directly and indirectly linked to epidemic diseases on Guam.

As some of the roots of cycad trees form a symbiotic relationship with cyanobacteria, a recent report from a team of American, Scottish and Swedish researchers proposed an explanation of the occurrence of BMAA in these plants, as their results indicated that BMAA is produced by cyanobacteria. However, they also reported that species of cyanobacteria giving rise to the characteristic blooming in oceans and lakes to a large extent produce BMAA, and that BMAA can be found in brain tissue of not only inhabitants of Guam but also of Altzheimer patients in North America. The present project was initiated since these observations could indicate that drinking water from surface waters could be contaminated with low levels of BMAA from cyanobacteria, and that drinkingwater could be responsible for a part of the incidence of degenerative neurological disease, including Parkinson and Alzheimer disease.

Information on BMAA was collected from literature identified by searching Food Science Technology Abstracts and Medline up to October 2006, using the search terms beta-methylaminoalanine (BMAA), Cycas, cycasin and cycad. Additional references were identified from the reference lists of identified literature.

The Project Group consisted of the following members:

- Ulla Beckman Sundh (co-ordinator), National Food Administration, Sweden

- Christer Andersson, National Food Administration, Sweden

- Johan Rosén, National Food Administration, Sweden

- Frode Fonnum, Institute of Preclinical Science, University of Oslo, Norway

- Ib Knudsen, Danish Institute for Food and Veterinary Research, Denmark

- Sari Sippola, Finnish Food Safety Authority, EVIRA, Finland

The present report has been prepared by Ulla Beckman Sundh ${ }^{1}$, Johan Rosén ${ }^{1}$, and Christer Andersson after thorough discussions in the project group, and finally adopted by NNT.

\footnotetext{
${ }^{1}$ National Food Administration, Box 622, SE-751 26 Uppsala, Sweden.
} 


\section{Summary in English}

Since the 1940's the cause of the highly raised incidence of amyotrophic lateral sclerosis (ALS) and Parkinsons dementia complex (PDC) within the local Chamorro population of Guam and neighboring islands of the south-west Pacific Ocean has been searched for. Even though the incidence now has decreased from its peak in the 1950's (179/100 000 for men and 61/100 000 for women) to numbers that compared to incidence in the Western world in the 1990s (1.9/100 000) are only a few times greater, the search for the main causative factor is still ongoing. Factors hypothesised and discarded after analysis with scientific scrutiny include hereditary causes, environmental causes, infectious agents, prions, and micronutrient deficiency. None of these have been thought to give the whole answer. The traditional diet was the only factor identified in epidemiological studies to be linked to the disease complex. In particular, the use of flour prepared form cycad seeds as a staple food by the Chamorro population became a topic of discussion in the 1960's. Since then it has been clearly demonstrated that there is a connection between dietary exposure to cycad and high incidence of ALS-PDC on Guam.

Cycad seeds contain several toxic principles, of which cycasin, a hepatotoxic and carcinogenic glycoside of methylazoxymethanol (MAM) is best known. As studies on experimental animals have shown that neurotoxicity is not the main effect of this compound, the search for another neurotoxin was initiated. In 1967 the amino acid beta-methylamino-Lalanine (BMAA) was isolated from the kernels of cycad, and a hypothesis presented that this amino acid could be the cause of delayed neurological disease. However, investigators have argued that the amount of BMAA that may be ingested when using traditionally detoxified cycad seed fluor in the diet is not enough to induce neurological damage. More recently, the theory of BMAA being the culprit got an upswing when it was suggested that BMAA may be biomagnified in the food chain. The investigators suggested that flying foxes, large bats that used to be a traditional Chamorro food, consume cycad seeds and store the compound as a free amino acid as well as linked to protein. Their studies revealed BMAA in formalin preserved museeum samples of flying foxes. The same researchers also reported that BMAA is produced in cyanobacteria of the genus Nostoc, isolated from the coralloid roots of the cycad where the cyanobacteria live in symbiosis with the plant. Based on this finding they put forward the suggestion that BMAA is produced by cyanobacteria, transferred to the symbiotic partner and subsequently transported within the cycad to different parts of the plant. 
Shortly after this hypothesis was launched, the same researchers that proposed the theory of biomagnification and identified BMAA in cyanobacteria published a couple of remarkable findings. Firstly they reported the occurrence of BMAA in brains of people that had died due to degenerative neurological disease, but not in brains of people that had passed away due to other causes. Later on they reported that BMAA to be found in 27 of 30 analysed strains of cyanobacteria, Since cyanobacteria are abundant and ubiquitous and occur in surface waters the implications for human health could be great.

The scenario of a potential exposure of the ordinary consumer in the Nordic countries to BMAA (via drinking water) initiated analytical work to identify BMAA and the production of the present risk assessment based on a literature review. A key issue is obviously a requirement of reliable analytical data. Without extraction methods, validated analytical procedures and instrumentation that can be relied upon, there is no possibility to test the theory of BMAA as a culprit for neurological disease. Early findings indicated that BMAA may be found both in free form and in a 'bound' form in the biological materials analysed. This observation needs confirmation and must be taken into account in the design of the analytical work. For material derived from cycads, several qualitative methods have been used, but no quantitative data of satisfying quality has been reported. Therefore all quantitative data on BMAA in cycad derived material must be used with care. For other matrices, all positive analytical findings have been based on only one analytical method identifying a BMAA derivative. In order to be able to draw reliable conclusions on the occurrence of BMAA in different matrices these findings need to be confirmed with another method, preferably a method with high specificity. Recent analytical studies in Sweden question the statement that BMAA is widely occurring in cyanobacteria.

From animal studies it is clear that ingestion of unprocessed cycad seeds may induce both acute and chronic toxicological effects. The toxicity of cycad seeds have long been well known and the Chamorro people have processed sliced seed material by washing in water when preparing cycad flour to reduce the quantity of the toxic principle. However, also products from cycad where the known toxicants have been reduced to very low or undetectable levels may induce toxic effects. It has been put forward that maybe a hitherto unknown toxicant in cycad may be responsible for neurodegenerative disease in Guam.

Several in vivo and in vitro studies have been carried out with BMAA as an isolated substance. These studies show that BMAA may induce toxic effects on the brain at high doses. They also indicate that with normal consumption it is unlikely that the amount of BMAA in processed cycad flour may be sufficient to result in toxic effects on the CNS and the degenerative neurological disease observed in humans native to Guam. On this ground, BMAA is unlikely to be the sole prime cause of degen- 
erative neurological disease in man, unless unknown mechanisms are confirmed that permit a higher degree of exposure to BMAA than is belived to occur today, or other mechanisms than are known today may mediate toxic effects.

In conclusion, the information available in published literature supports a true connection between cycad exposure and the development of ALS-PDC on the island of Guam. However, it is not established which the toxic compound(s) in the cycad are. One candidate is BMAA, which has been found in the cycad by several investigators, using different methods of analysis. Unfortunately, there are sufficiently validated methods to quantify BMAA., This situation makes it difficult to establish or repudiate a relationship between BMAA exposure and neurological disease. In experimental studes, BMAA has been found to have acute toxic effects on the brain, but only at very high concentrations. Human epidemiological data and information from animal in vivo studies show that the amount of BMAA available in processes cycad flour is not enough to cause neurodegenerative disease after dietary exposure.

The recently published hypothesis that BMAA is widely occuring in cyanobacteria at levels that would present a human risk factor for development of neurological diseases is strongly questioned by recent analytical studies. Accordingly, also the extensive exposure of human beings to BMAA via cyanobacteria in drinking water, and the associated human health risks, are questioned. Therefore, at the present state of knowledge, there are no requirements to induce measures to reduce the BMAA exposure in the Nordic countries. 



\section{Introduction}

Is it possible that the cause of degenerative neurological disease such as amyotrophic lateral sclerosis (ALS), Parkinson dementia and Alzheimers disease could be a dietary exposure to an amino acid not known to occur in proteins? This is a question that has to be tackled by authorities after a suggestion in 2005 that the potentially neurotoxic amino acid BMAA might occur in drinking water.

In April 2005 Cox and co-workers published data indicating a wide occurrence of the compound $\beta$-methylamino-L-alanine, BMAA, in cyanobacteria (blue-green algae). BMAA was reported to be formed by cyanobacteria in symbiotic relationships with plants, as well as in freeliving cyanobacteria forming blooms in fresh, brachish and marine waters.

$\beta$-Methylamino-L-alanine (BMAA) was identified in biological material by Vega and Bell in 1967. The compound was originally isolated and characterized in kernels of the cycad Cycas circinalis. The chemical identification was confirmed by synthesis of $\beta$-methylamino-L-alanine and by comparing the synthesized material with the isolated material. Cycads are among the most primitive living seedplants found today. They are palmlike plants belonging to the family Cycadaceae, and are often termed living fossils because their morphology has changed very little from that of ancestors in Mesozoic times, 250-65 million years ago (Fosberg, 1964). Once found throughout the world they are now restricted to warm climates in the New and Old world. Much of the endurance of the cycads may be ascribed to the biosynthesis of a variety of protective, secondary compounds. These toxicants seem to be crucial elements in the development and maintenance of mutualism (pollination) and parasitism (herbivory) by cycad-linked herbivores (Schneider et al., 2002). The ubiquitous occurrence of these secondary compounds in all extant cycad genera strongly suggests that these constituents evolved early in the history of these plants (Schneider et al, 2002).

The reason investigators started to analyse cycads was that the American medical staff connected to a military base established on the island Guam during the second world war, noticed that the incidence of degenerative neurological disesase, particularly amyotrophic lateral scerosis (ALS) and a type of Parkinson dementia, were more than hundred times more common on the island than elsewhere. Furthermore, early epidemiological studies linked the traditional diet of the local population to a high incidence of neurological disease. Central in the diet was flour prepared from the local cycad trees. 
During investigations on the distribution patterns of BMAA in various tissues of Cycas micronesica, a cycad endemic to the island Guam, Banack and Cox (2003b) noted that BMAA occurred in morphologically specialized, positively geotropic "coralloid" roots of the plant but not in unspecialised, negatively geotropic roots. This finding stimulated a search for the ultimate source of BMAA in cycads. In contrast to the negatively geotropic roots, the morphologically specialized, positive geotropic "coralloid" cycad roots harbour endosymbionts, which are nitrogen-fixing cyanogenic bacteria. These cyanobacteria live in the coralloid roots, invading a specialized region of the root. It turned out that BMAA levels varied with root maturity. Uninfected coralloid roots contained no BMAA in the study of Banack and Cox (2003b), coralloid roots with new flourishing infection contained $37 \mu \mathrm{g}$ free BMAA per g root, and coralloid roots with abundant but senescent infections around $2 \mu \mathrm{g}$ free BMAA per $g$ root. To determine whether the BMAA was produced by the Cycad root as a response to the symbiotic interaction, or by the cyanobacteria occurring in the roots, the cyanobacteria were isolated for in vitro studies. The isolated cyanobacteria from coralloid roots of $C$. micronesica were identified as belong to the genus Nostoc, and could be grown in vitro. When grown in exenic culture they produced $0.3 \mu \mathrm{g}$ free BMAA per g bacteria according to Cox and co-workers (2003).

This finding stimulated the investigators to search for BMAA also in other plants where cyanobacteria are symbionts: Azolla filiculoides, a floating fern with cyanobacteria symbionts in its leaves, contained $2 \mu \mathrm{g}$ free BMAA per g plant, and Gunnera kauaiensis, a large-leafed angiosperm with cyanobacterial symbionts in the petiolar tissue, contained 4 $\mu \mathrm{g}$ free BMAA per g plant. From these observations, Cox et al. (2003) drew the conclusion that BMAA is not produced by the cycad itself but by the cyanobacteria present in the collaroid roots. It was proposed that BMAA-producing cyanobacteria might have a dual role for the cycad. They could produce useful antiherbivory compounds, and at the same time fix nitrogen and supply additional material of this limiting mineral nutrient for the tree.

If this hypothesis is correct, BMAA is obviously transferred from the Nostoc cyanobacteria to the cycad symbiont, and translocated from the root to other parts of the tree, including the kernels.

Cox et al. (2005) expanded on their studies on cyanobacteria by analysing for BMAA in cyanobacterial species from all five sections of this organism group, as well as in additional cyanobacterial symbionts isolated from lichen and a diversity of plant species. Analysis of Nostoc strains isolated from symbiotic relationships with lichen and host plants of broad taxonomic diversity (hornwort, liverwort, three cycads, and five Gunnera species) indicated that $73 \%(8 / 11)$ of these strains produced BMAA. Among free-living cyanobacteria, BMAA is produced by members of all five cyanobacterial sections, in $95 \%(20 / 21)$ of the genera 
tested and 97\% (29/30) of the strains tested. Thus, BMAA is found in cyanobacteria from various geographical regions and diverse environments throughout the world. The data, however, show a very wide variation in BMAA content between samples, species, and genera.

Because of the widespread occurrence and importance of cyanobacteria, Cox et al. (2005) concludes that it is likely that human populations far from Guam may be exposed to the potentially neurotoxic BMAA through drinking water or specific foods that have accumulated BMAA through the food chain. This report aims to address the question whether degenerative disease outside Guam might be the result of exposure to this rare amino acid. 



\section{A historical perspective on the link between BMAA exposure and degenerative neurological disease}

In the aftermath of World War II, U.S. Army physicians assigned to Guam, found the local Chamorro people to be afflicted with a progressive neurodegenerative disease described as amyotrophic lateral sclerosis (ALS) or an ALS-like condition appearing hand-in-hand with an unusual form of Parkinson dementia (PD). The incidence of amyotrophic lateral sclerosis-Parkinson dementia complex (ALS-PDC) was estimated to be 50 to 100 times higher than elsewhere in the world (Zimmerman, 1945; Koerner, 1952; Arnold et al., 1954, Kurland and Mulder, 1954; Hirano et al., 1961a). After recognizing the high incidence of ALS-PDC on Guam, the US National Institute of Neurological Disorders established a registry for cases of motor neuron disease and Parkinson dementia complex on the island Guam in 1956. This registry has been a goldmine for epidemiological studies of the disease. Epidemiological studies focusing on ALSPDC on Guam are summarised in Annex 1 of this report.

The registry on ALS-PDC cases on Guam revealed that the average age adjusted annual incidence of the disease complex peaked at 179/100 000 for men in 1959-61 and 61/100 000 for women in 1956-58. This incidence rate should be compared to 1-2 ALS cases/100 000 individuals, and similar rates for PDC in the white population elsewhere in the world. Worms (2001) estimated the annual incidence for motor neuron disease, including ALS, elsewhere in the world to 1.89/100 000 in the 1990's. In the period 1960-1970 it had been 1.3/100 000. The increase in incidence seemed to be related to countries in Southern Europe, to women, and to persons above 75 years of age. The increased ALS incidence was asssumed to be linked to a longer survival and, possibly, better diagnostic tools. However, Worms (2001) could not exclude a true increase in incidence. A steady downward trend in prevalence of both disorders has occurred on Guam after peaking nearly sixty years ago, the prevalence of ALS declining quicker than the prevalence in PDC (Román et al., 1996; Poorkaj et al, 2001). In 1989 the annual age-adjusted incidens for ALS in Guam was 7/100 000 and for PD 22/100 000. Thus, in present time the incidence is a few times more common among Chamorros on Guam than among people elsewhere (Figure 1). Warning et al. (2004) indicated that the reason for the slower reduction in PD incidence rate could be that this 
degenerative neurologic disorder peaked later than ALS, 1960-1964 for men, and 1970-1979 for women.

In parallel with the reduced annual incidence rate during the period 1941 to 1985 the age of onset of the diseases ALS-PDC in Guam increased from 42 to 56 years for men and from 42 to 55 years for women, which could indicate that the exposure to the risk factor has decreased substantially, or perhaps even ceased to occur (Román et al. (1996). Poorkaj et al. (2001) differentiated between the two diseases and concluded that the age at onset for ALS had increased from 47.6 to 51.9 years between 1950's and 1970-1980, and from 42.1 to 52.2 years for PDC. The disease now mainly occurs among older adults and rarely in any individuals born after 1960 .

\section{Ruling out inheritance as the major cause of ALS-PDC}

As ALS was more common on Guam and on neighbouring islands of the Mariana islands than in most other places of the world, and also more common in some villages in Guam (the highest prevalence, 250 per 10 0000, was found in Umatac), and more common in some families than in others, a genetic cause of the disease was initially favoured. However, epidemiologists have until recently been unable to find any evidence of a genetic cause to the disease (Kurland and Mulder, 1954; Garruto and Yase, 1974; Chen et al., 1996). Roman et al. (1996) reports on the family history for 303 Chamorros who developed motor neuron disease in Guam between 1956 and 1987. The risk of development of motor neuron disease in susceptible brothers and sisters was up to 28 times greater than the general population lifetime risk. Although the epidemiology of the disease was inconsistent with Mendelian inheritance, the investigators found it conceivable that a susceptibility gene could be present among the Chamorros. Examples of alterations that have been investigated include modified apolipoprotein E (hypothesised to have a role in the NFT formation and constitute a genetic risk factor of Parkinson's diseasee), CYP2D6 (results in reduced rate of metabolism of exogenous toxins), and the tau protein (mutations in the tau gene cause autosomal dominant frontotemporal dementia) (Chen et al., 1996; Poorkaj et al., 2001). Presently, it is investigated whether a variant melastatin allele may confer vulnerability to the disease (Hermosura et al., 2005). 


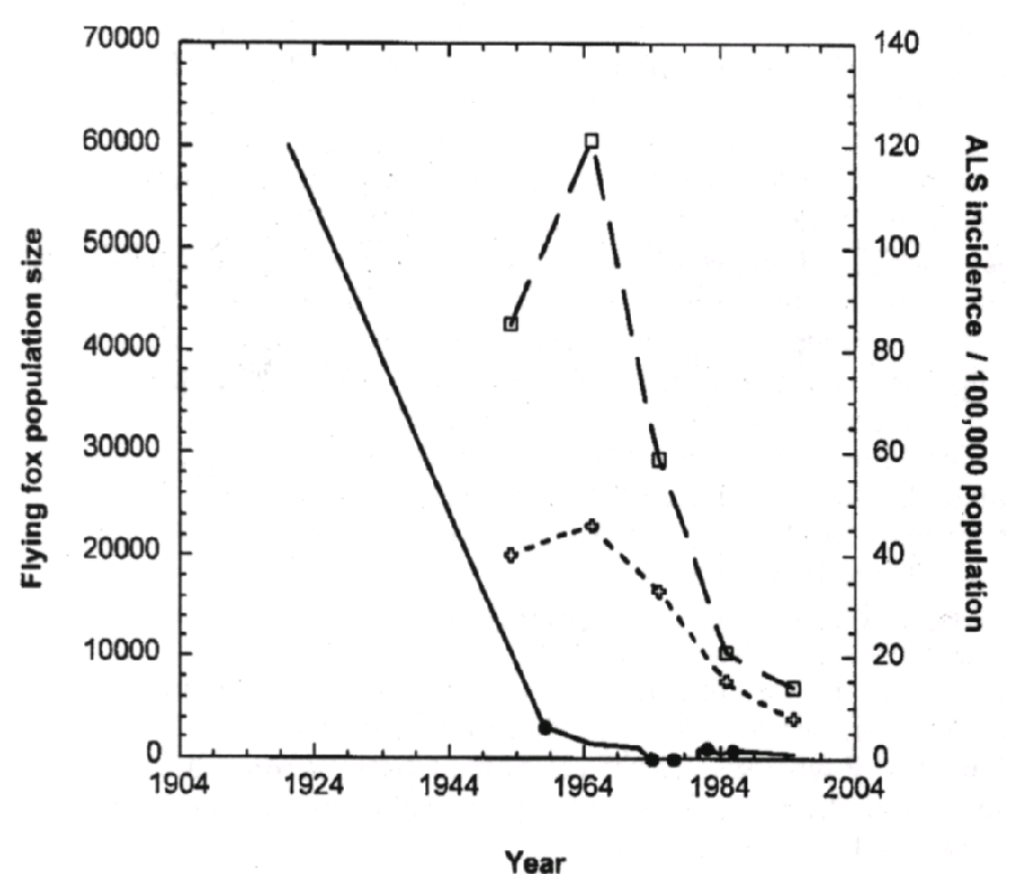

Figure 1.The incidence of ALS-PDC among Chamorro people on Guam and the number of flying foxes on Guam. Filled circle = flying fox population size; open box $=A L S$ incidence in males/100 000 males; open plus sign = ALS incidence in females/100 000 (Cox et al, 2002).

In 1958 two prospective studies consisting of ALS and PDC patients and controls were started in Guam. After 25 years, in 1983, it was clear that there was a significantly higher risk for ALS and PDC among parents, siblings and spouses of patients than among relatives of controls without symptoms (Plato et al., 2002). Also in the follow up after 40 years, relatives, siblings and wives to ALS patients, but not husbands to ALS patients, had a higher risk for ALS or PD than Chamorro people in general. Relatives to the control group did not have an augmented risk for the diseases. Some categories of relatives to PD patients had a higher risk for both ALS and PD than the population in general, and higher risk for PD than Chamorro in general. The most important finding was that children to patients with PD had an increased risk for ALS and PD, but children to patients with ALS did not have an increased risk to acquire the diseases (Plato et al, 2002; Waring et al., 2004). Poorkaj et al. (2001) concluded that the disease clusters in families, indicates the importance of inheritance for ALS-PDC susceptibility, whereas the change in men to women ratio (2.5 to 1.5$)$ and incidence over time and corresponding change in onset age suggests that gene-environment interactions are important. (Roman et al., 1996; Poorkaj et al., 2001). 


\section{Searching for an environmental cause for ALS on Guam}

By the end of 1987 no Chamorro born on Guam after 1949 had developed motor neuron disease. The longest latency or incubation period found was 34 years: This was a Chamorro, who had been away from the island and returned to Guam and developed motor neuron disease. The shortest incubation period was 3 years, found in a Filipino who lived in Guam 36 months before developing the disease. In general the latency period to acquire the disease increased from the middle of the 1900's to present time (Román et al., 1996). Also this observation gave more support to environmental factors than to genetic factors being important for ALSPDC development on Guam.

The fact that Chamorro migrants from Guam to the mainland United States could develop ALS-PDC 1-34 years later, suggested that exposure to environmental factors during early adolescence may be sufficient to cause disease 30 years later (Worms et al., 2001). Similarly, Román et al. (1996) report that among Chamorro migrants who left Guam between 1934 and 1966 there were 41 cases of motor neuron disease and only 6 cases among later migrants. The conclusion was drawn that 1960-66 seemed to be the most recent years of meaningful risk for acquiring motor neuron disease on Guam.

Evidence for risk factors to ALS-PDC on Guam and the neighbouring island Rota was also obtained from migrants to Guam. Reed et al. (1987) observed a higher than expected occurrence of both ALS and PD in Filipino migrants to Guam, as long as 29 years after their arrival. Román et al. (1996) noted that of six Filipino and three US white men who arrived to the island and subsequently developed motor neuron disease, seven arrived in the late 1940s, one in 1956 and one in 1963. Since 1960 no new cases of motor neuron disease occurred in Filipino migrants followed for their health development until 1987. These observations confirm that exposure during adulthood would be sufficient to aquire the disease, and indicated that a long latency period is involved.

The absence of the diseases among US military and construction workers and among a small community of Caroline Islanders on Guam suggested that exposure must be longer or more intimate than that of these visitors and migrants, or that genetic susceptibility was involved.

An early epidemiological study performed on Chamorro people living on Guam and Rota was published by Reed and co-workers in 1968. In this study, a baseline medical examination and interview was performed with 899 Chamorros on Rota and Guam. One member of at least 20 years of age from a random selection of one of every 20 households from each town on Guam, and from the total population of Rota was approached. 628 persons on Guam (74\% of those approached), and 337 of the persons from Rota $(80 \%)$ accepted to participate. The interview included collection of information on traditional Chamorro habits, the history of medical problems, and the use of traditional Chamorro and available western 
foods. In the investigated population, five prevalent cases of ALS-PDC were diagnosed in 1969. In a follow-up 15 years later, in 1983, four new confirmed cases of ALS, one with ALS-PDC and 23 with PD were identified among those persons that could be traced (Reed et al., 1987).

As ALS-cases were to few for a statistical comparions, only the baseline information on PD cases were compared with the baseline information of individuals who had not developed degenerative neurological desease. Age-adjusted mean values of 23 selected baseline variables were compared including systolic and diastolic blood pressure; serum glucose, cholesterol, uric acid, triglyceride; weight; disease history of diabetes, stroke, asthma, arthritis, kidney disease, hypertension, mental disorder, alcoholism; smoking habits, years lived abroad, years of education, employement/occupation, traditional attitude score; Chamorro language skill; traditional food score (detailed definition not given); and stress symptom score. Of these 23 variables preference for traditional foods was the only significant variable associated with PD incidence. In addition, age was associated with PD incidence.

\section{Infectious agents an unlikely cause of ALS-PDC on Guam}

Similarities of the degenerative neurological disease with postencephalitic Parkinsonism resulted in speculations that the condition had an infectious origin. But also this theory had to be abandoned as no evidence of an encephalitic infection having occurred on the island was found (Gibbs and Gajdusek, 1982). Neither was it possible to link any unusual infectious agents, such as prions, to the disease (Sacks, 1993).

\section{Ups and downs for the hypothesis of a dietary cause of ALS-PDC on Guam}

Whiting (1963) initiated ethnobotanical studies to reveal whether the Chamorro lifestyle and diet might be implicated in the development of ALS-PDC on Guam. The culture of the Chamorro population on Guam was more or less uninfluenced by other cultures until the Spaniards reached the island in the $16^{\text {th }}$ century. Since then, over 400 years of colonial ruling has influenced Chamorro culture extensively, in particular during the last sixty years. Today, Guam is a U.S. territory with several military bases. Currently, the Chamorro people comprise $<50 \%$ of the population of Guam. In the absence of a clear hereditary explanation, Kurland stimulated a continued search for a dietary factor. During four cycad conferences held between 1962 and 1972 cycad seeds were suggested as a possible culprit (Whiting, 1988).

In the Chamorro lifestyle two environmental causes for the high incidence of ALD-PDC in the Pacific region has been discussed more in depth; one hypothesis, which now has poor support, is related to a dis- 
torted mineral metabolism leading to increased intestinal adsorption of aluminium, the other hypothesis is related to the occurrence of a neurotoxin in the diet. Both these theories could agree with the epidemiological finding reported above that the only risk factor for ALS-PDC on Guam and Rota identified among 23 investigated factors was the preference for traditional Chamorro food (Reed et al., 1987).

The hypothesis that ALS-PDC development is related to a dietary deficiency of minerals emanate from the findings of Yase in 1972 and Gajdusek and co-workers in the 1980's that CNS tissue from ALS-PD cases contained increased levels of aluminium, mangan and cadmium (Yase, 1972, 1980; Gajdusek and Salazar, 1982; Gajdusek, 1985). This observation lead to the hypothesis that chronic dietary deficiencies of calcium and magnesium could induce secondary hyperparathyoridism, leading to increased absorption of toxic metals such as aluminium, and deposition of these metals along with calcium and silicon in neurons. In the study of Román et al. (1996) referred to above, the investigators found no correlation between the risk of developing motor neuron disease and mineral (calcium, magnesium or aluminium) levels in soil and water, except for a significant correlation between disease risk and iron concentrations in water. Zhang et al. (1996) investigated the hypothesis by comparing the geographical pattern and incidence of ALS-PDC with the geology of Guam and found no significant correlation with concentrations of calcium, magnesium, aluminium or manganese in water and soil. The incidence rates were, however, significantly correlated with iron and silicon (men only) in drinking water and soil levels of cobalt and nickel. Absence of a correlation between calcium intake and ALS-PDC is supported by no correlation being found also between the profile of ALS-PDC distribution on Guam and the profile of chronic calcium deficiency in the same population (Zhang et al., 1996).

However, Durlach et al (1997) pointed out that nutritional Ca and $\mathrm{Mg}$ deficiencies in the study of Zhang et al. (1996) had only been inferred from indirect data (low concentration in garden soils and river water), and not from dietary intake. Therefore, Ahlskog et al. (1997) evaluated Ca, $\mathrm{Mg}$ and heavy metals status in 12 patients with ALS-PDC and 12 healthy Chamorro subjects. Patients had normal values for $\mathrm{Ca}$ in serum and urine. No patients had reduced $\mathrm{Mg}$ and $\mathrm{P}$ values in serum but a few patients had low urinary $\mathrm{Mg}$ concentrations. Among metals ( $\mathrm{Al}, \mathrm{Mn}, \mathrm{Pb}, \mathrm{Cd}, \mathrm{Cu}, \mathrm{Fe}$, $\mathrm{Hg}$ and $\mathrm{Zn}$ ) analysed in serum and urine, all were statistically similar in the patient and control groups except for a slight elevation of $\mathrm{Pb}$ in blood in the patient group. Concentrations of heavy metals in hair and nails were similar in the two groups. Taken together, there was no evidence in these data to support a major role of $\mathrm{Ca} / \mathrm{Mg}$ deficiencies combined with heavy metal loads in the development of Western Pacific ALS-PDC (Durlach et al, 1997; Kurland et al, 1994). 
Furthermore, a role for mineral deficiency in the development of ALS-PDC on Guam is negated by a neuropathologic study of neurofibrillary degeneration of selected neurons in brains of 69 deceased Chamorro natives of Guam without known clinical evidence of ALS, PD or dementia (Reed et al., 1987). This study revealed a high prevalence of neurofibrillary degeneration, $29 \%$ in persons aged $30-39$ and $100 \%$ in persons aged $80-89$. The prevalence rates were $70 \%$ both in the southern villages with low calcium levels and the northern and central villages with normal calcium levels (Reed et al, 1987).

\section{Identification of cycad flour as a risk factor for ALS-PDC}

A study of the traditional Chamorro diet identified the use of flour prepared from seeds of a local tree on Guam, the cycad Cycas circinalis ${ }^{2}$, as a risk factor for ALS-PDC (Whiting, 1963). Cycads are among the most primitive living seedplants found today. They are palm-like plants belonging to the family Cycadaceae, and are often termed living fossils because their morphology has changed very little from that of ancestors in Mesozoic times 250-65 million years ago (Fosberg, 1964). Once found throughout the world they are now restricted to warm climates in the New and Old world. Much of the endurance of cycads has been ascribed to the biosynthesis of a variety of protective, secondary metabolites. These protective compounds seem to be crucial elements in the development and maintenance of mutualism (pollination) and parasitism (herbivory) by cycad-linked herbivores (Schneider et al., 2002). The ubiquitous occurrence of carcinogens and other toxicants in all extant cycad genera strongly suggests that these constituents evolved early in the history of these plants (Schneider et al, 2002). Cycas circinalis are common on Guam and grow as large forests. The seeds of the cycad are used for flour production, a staple food for the Chamorro in earlier times. The Chamorro have been well aware that the seed are acutely toxic, and detoxify them before use.

Also native herbivorous mammals (for instance kangaroos in Australia) feed in their natural habitat on local cycads (leaves) but seem not to suffer. African birds and hyraxes eat the pulp of cycad stems, and elephants consume cycad cones. The question is put whether this tolerance in endemic species is due to detoxification or to avoidance of risky overdoses.

In the light of history, suspecting the cycad to be responsible for the neurological degenerative diseases in Guam is not far-fetched. Leaves of

\footnotetext{
${ }^{2}$ Cycas circinalis and Cycas rumphii are by some taxonomists held as separate species or varieties, closely related to C. micronesica, and by other botanists claimed to be the same species. Hill (1994, 1995) excludes both Cycas circinalis and Cycas rumphii from Guam and has erected the new binomial species C. micronesica, placed in the Cycas rumphii species complex, for the indigenous cycad species of Guam and neighboring islands.
} 
various cycads are eaten by domestic animals. Plants from six of nine genera of Cycads have been implicated in the development of toxic symptoms in livestock. Sheep and cattle introduced to Australia have been reported to fed on cycads during periods of food shortage and have suffered badly.

Early investigations on the chemical composition of cycad leaves revealed the presence of glycosides of methylazoxymethanol, best known as cycasin (Riggs, 1956; Nishida et al., 1955), a compound that exerts hepatotoxic and carcinogenic properties when fed orally to experimental animals. The active compound is the aglycone, produced when the parent glycoside is split of by the action of enzymes produced by the bacteria in the animal gut. Interestingly, sheep, cattle and horses that have fed on cycad leaves have been reported to develop irreversible neurological paralysis of the hindquarters, a staggering gait (Mason and Whiting, 1966), which resembles lathyrism in humans that have consumed seeds of the beans Lathyrus sativus, Lathyrus cicera or Lathyrus clymenum. Lathyrism is a neuronal disease caused by toxic non-protein amino acids, such as $\alpha$-amino- $\beta$-oxalylaminopropionic acid, occurring in these beans (Adiga et al., 1963; Murti et al., 1964; ICARDA, 1995; Schneider et al, 2002). The fact that hepatotoxic and neurological effects frequently did not occur simultaneously, stimulated the search for other toxins than cycasin in cycad plants, and the evident compounds to look for were toxic amino acids.

The search was successful when Vega and Bell in 1967 isolated and characterized the compound BMAA from kernels of Cycad circinalis. The chemical identification was confirmed by synthesis of $\beta$ methylamino-L-alanine and comparing the synthesised material with the isolated material, and the neurotoxicity by giving the compound to chickens (Vega and Bell, 1967; Vega et al., 1968). Only the L-isomer occurred in the cycad, and only the L-isomer produced toxic effects when injected in comparable amounts into chicks and young rats (Vega et al., 1968).

As a consequence of BMAA being identified in cycad seeds, Kurland (1972) presented a new hypothesis for the local appearance of ALS-PDC, namely that it was a delayed consequence of the ingestion of cycad neurotoxin from cycad flour. During the Japanese occupation of the early 1940s, the seeds of C. circinalis proved an important dietary staple for the Chamorros of Guam. However, Kurland's hypothesis was not fully explored at the time, as studies on experimental animals fed the cycad seeds or flour were unable to demonstrate the induction of neurological disease at the doses used (Dastur, 1974).

\section{Other hot spots for high ALS-PDC incidence}

Geographical hot spots for the occurrence of progressive neurogenerative disease of the ALS-PDC type was later on described also among residents 
of the northern Kii Peninsula on Honshu, Japan (Yase, 1979), and among the Auyu and Jaqai linguistic groups of the southern lowlands of west New Guinea, Irian, Java, Indonesia (Garruto, 1986). In all these regions the incidence of ALS-PDC have earlier been much higher than at present. The significant decline in risk induced an intensive search for a disappearing environmental factor, including mineral excess or deficiencies, prions, and dietary factors (Garruto et al., 1984; Yase, 1987; Rowland, 1991; Sacks, 1993). Although it was originally believed that the neurological disease in this case could not be due to inherent toxicants in cycad, as these populations did not consume cycad flour or animals feeding on cycads (Gajdusek and Salazar, 1982), this hypothesis was revitalized when it was found that these Western Pacific isolates with high ALSPDC incidence indeed were exposed to cycads.

Whereas cycad flour from Cycas circinalis is consumed in Guam, seeds of this plant species are not consumed in the Kii peninsula of Japan and in Western New Guinea. However, raw cycad seeds of Cycas revoluta are used in medicine in the Kii peninsula (Spencer et al., 1987c), and cycad poultices of Cycas media are topically used in the affected regions of western New Guinea (Spencer et al., 1987b). Thus, cycad seeds may still be the link between the three geographical areas affected with increased incidence of neurodegenerative disease, but it does not have to be their dietary use (Spencer, 1989). A calculation of the cumulative dose of BMAA after topical application of unwashed cycad seed poultices once a day for 1 month resulted in a theoretical exposure approximately ten times the exposure to free BMAA in the diet (10.8 as compared to 1.8 $\mathrm{mg} / \mathrm{kg} / \mathrm{month}$ ) (Duncan, 1991). However, there is at present no experimental information on whether the bioavailability of BMAA differs between the topical and dietary routes of exposure, and it is therefore not possible to evaluate the validity of the calculation made by Duncan (1991). There is no information on how the cycad seeds were treated, if at all, before preparation of the poultices and there is no information on the conditions when the topical poultices were used, the extent and frequency of use and whether they were used on undamaged or damaged skin. Furthermore, as there is no information on the formulation of the poultices, it is not possible to assess to what extent the water-soluble amino acid BMAA would be able to penetrate the skin and enter the bloodstream.

\section{Plant foods and degenerative neurological disease}

Cycad constituents became even more interesting as cause of ALS-PDC when Spencer et al. (1987a, 1987d) showed that dietary exposure to BMAA cause selective degeneration of motor neurons in the cerebral cortex and spinal cord of macaques (Macaca fascicularis), sometimes in synchrony with parkinsonian features and behavioural changes. These observations were in line with the observation that neuroexcitatory non- 
protein amino acids ((-)-( $\beta$-N-oxalyl)- $\alpha, \quad \beta$-diaminopropionic acid (BOAA), $\beta$-(N- $\gamma$-glutamyl)-aminopropionitrile, $\alpha, \gamma$-diaminobutyric acid, lathyrine, homoarginine and $\gamma$-hydroxyhomoarginine) occur in seeds of various Lathyrus species used as food mainly by the poorer population in some areas of India and Africa. The observations also indicate that this dietary exposure was linked to the risk of developing the severe neurodegenerative disease neurolathyrism (Murti et al., 1964). However, contrary to the human situation, the disease found in monkeys was acute and reversible. For humans to consume the amount of BMAA resulting in similar neurological effects to those observed in monkeys would be nearly impossible, as it would require the consumption of massive amounts of cycad seeds on a daily basis; based on the original belief that BMAA occurs only in limited amounts in cycad flour (Gajdusek, 1985; Duncan et al., 1990). The potential link between BMAA exposure and development of ALS-PD was enforced by the observation that a cynomologus monkey given a large amount of BMAA $(>100 \mathrm{mg} / \mathrm{kg})$ developed motor neuron degeneration and dysfunction and parkinsonian features (Spencer et al., 1987a, 1987d). Notably, lowered incidence rates of ALS-PD parallels a reduced exposure to cycads on Guam as new sources of food and medicines became available on the Mariana islands after the Second World War and the native Chamorro people acculturated to modern ways.

\section{Identifying the neurotoxin in cycads}

The evaluation of the hypothesis that BMAA in cycad seeds directly or indirectly is responsible for the high incidence of ALS-PDC on Guam is complicated by the fact that cycads contain several toxic compounds. Most notably, these plants contain the carcinogens cycasin $(0.10 \%$ of f.w.), which is an amino sugar methylazoxymethanol $\beta$-D-glucoside, and methylazoxymethanol (MAM), the aglycone of cycasin. It is known that substantial amounts of these compounds can remain in washed cycad flour, depending upon the routine used (Spencer et al., 1991a). A new theory based on a "slow toxin" damaging DNA in certain vulnerable neurons was suggested (Spencer et al., 1991b). Many years after the BMAA intake the neurons might be subjected to a degenerative process that would lead to the changes characteristic of ALS-PDC. Support for such a hypothesis was obtained in rat studies with methylazoxymethanol (MAM), the aglycone of cycasin (Esclaire et al., 1999). Earlier studies had shown that incorporation of ground cycad seeds into rat feed induced developmental abnormalities of the nervous system of the animals (Campbell et al., 1966).

Cycad seeds also contain the toxic compounds pakoein, macrozamin $(0.42 \%$ fresh weight; Whiting, 1963$)$, and possibly the toxic non-protein amino acid $\beta$-N-oxalylamino-L-alanine (BOAA) (Charlton et al., 1992; Oh et al., 1995; Li et al., 1996). More recently, Khabazian et al. (2002) 
isolated and identified putative active neurotoxic components of washed cycad chips. The three new compounds were all sterol $\beta$-glucosides $(95 \%$ $\beta$-sitosterol $\beta$-D-glucoside, $4 \%$ stigmasterol $\beta$-D-glucoside, and $1 \%$ campesterol/dihydrobrassicasterol $\beta$-D-glucoside) present at a total amount of $21-155 \mathrm{mg} / \mathrm{kg}$ dry weight. In washed chips no or very low levels of cycasin, methylazoxymethanol (MAM), $\beta$-N-oxalyl- $\alpha, \beta-$ diaminopropionic acid (BOAA) (up to $0.14 \mathrm{mg} / \mathrm{kg}$ dry weight) and BMAA (up to $3 \mathrm{mg} / \mathrm{kg}$ dry weight) were found. As shown by Khabazian et al. (2002) these sterol $\beta$-D-glucosides appears to have distinct effects on NMDA receptor activation, as NMDA antagonists can block their various actions in vitro without competing for binding to the receptor. The actions include induction of depolarising field potentials resembling those of NMDA, activation/inhibition of some of the same protein kinases influenced by NMDA, and the release of glutamate and LDH. As a consequence, these sterol glucosides are acutely toxic to cells in the adult rat cortex. Furthermore, synthetic $\beta$-sitosterol $\beta$-D-glucoside (but not the aglycone) was shown to be toxic to cells in slices of adult cerebral cortex and to foetal astrocytes in culture. Although studies performed on single cells did not support the hypothesis of an action via the NMDA receptor, these deviating observations could be explained. Therefore, the authors found it highly probable that the observed effects are caused by indirect actions of glutamate released in the tissue, acting ultimately via NMDA receptor activation. Wilson et al. $(2002,2003,2004,2006)$ have reported data on mice fed cycad seeds containing $\beta$-sitosterol glucosides, which showed the mice to respond with progressive motor, cognitive and olfactory function deficits combined with neuronal losses in the appropriate neural subsets (behavioural and neuropathological outcomes). Altogether these observations indicate that cycad consumption may be an important factor in the ethiology of ALS-PDC and further suggest that some sterol glucosides may be involved in neurodegenerative disorders. Thus, instead of explaining the potential effects of BMAA, these studies introduce a new candidate for ALS-PDC on Guam. However, this report will focus on the potential dietary toxicity of BMAA.

\section{Biomagnification of BMAA and the theory of a neurotoxic reservoir}

For a long time it was belived that BMAA only occurred in free form. Because of the difficulty to explain a "slow toxic" action of water soluble free BMAA molecules on the neurological system, Murch et al. (2004a) suggested that the molecules may become incorporated into proteins as ordinary amino acids, or bound to proteins in another way. In this bound form they may function as an endogenous neurotoxic reservoir that slowly releases neurotoxin directly into the cerebral tissues through protein metabolism. As described in the chapter on occurrence, higher levels of BMAA was reported in bound form than occurred in free form. Thus 
exposure to BMAA through the cycad flour could be substantially higher than originally believed, but still too low to explain the degenerative neurological conditions descdribed in ALS-PDC patients.

A dietary cause for the neurodegenerative diseases in Guam have recently received much stronger support when Cox and Sachs (2002) proposed a route for possible biomagnification of cycad toxins. Cox and Sachs pointed out that the Chamorro population of Guam ingested large quantities of cycad toxins indirectly by eating flying foxes, a type of fruit eating bats living on the island. The Chamorros consumed flying foxes on ceremonial occasions and at special social gatherings, and the habit was an integrated part of the Chamorro culture, particularly in the Southern regions of the island. It was also in these regions the highest incidence of ALS-PDC was recorded (Zhang et al., 1996).

The two flying fox species, Pteropus tokudae and P. mariannus, which lived on the island could sometimes consume up to two and a half times their body weight per night in fruit and nectar (Dobat, 1985). Cycads are included among the different plants used as food sources by the bats who squeeze out the juice from the sarcotesta and then spit out the pulp. Cox and Sachs (2002) suggested that the flying foxes could biomagnify the neurological toxins in cycads. Bats, like other mammals, are known to accumulate other toxic molecules in their fat. Thus, habitual consumption of Guamanian bats by the Chamorro people could have resulted in a significant ingestion of cycad toxins, resulting in much higher exposure than was earlier believed to occur only from the cycad flour.

According to Cox et al. (2002), the time course for the incidence of ALS-PDC in Guam interestingly follows the history of the flying fox population on Guam (Figure 1). However, the validity of this statement has been questioned. The slope in the graph that shows the decline in flying foxes on Guam depends solely on one point on the graph, i.e. the estimate of the size of the population in the 1920's. This estimate is derived from population estimates on nearby islands in the early 1900's, combined with historical records of forest cover on Guam (Cox et al., 2003), which Miller (2006) considers to be a shaky foundation.

Originally the foxes were catched by net and other simple equipments for the ritual feasts. This was not an easy task. When the Americans in the 1940's built up their Army bases on the island, heavily improving the access to firearms, the hunter yields of flying foxes increased and a new scenario was introduced. Commercial hunting of the flying foxes began in the second half of the 1960's and peaked in the mid to late 1970's (Lemke, 1992). Pteropus tokudae had been hunted into extinction by 1978 , and the previous large populations of $P$. mariannus were reduced to less than 100 animals by 1974 (Wiles and Payne, 1986). When the Chamorros were forced to buy imported flying foxes, these were species coming from Samoa and elsewhere, which did not feed on cycads con- 
taining the neurotoxins. Thereby, the exposure to BMAA was substantially reduced. The high rates and subsequent decline of ALS-PDC in Guam (Kurland and Mulder, 1954; Steel and Guzman, 1987; Zhang et al., 1996) mirrored the rise and eventual decline in consumption of cycad-fed flying foxes by the Chamorro people (Monson et al., 2003).

Cox and co-workers (2003) suggested that the traditional consumption pattern of Chamorro people resulted in a high enough BMAA exposure (due to biomagnification) to be interesting as a natural toxin. According to the bioagnification theory, BMAA is synthesized by the cyanobacteria in collaroid cycad roots, accumulates through the Guam food chain (cycad seeds, flying foxes) and is then inadvertently ingested by the Chamorro people during their traditional feasting on flying foxes, and/or taken up from local medicines or poulties. Hunter's yield of flying foxes in pre-European contact times were likely low and success was infrequent. Netting animals in trees at night time or snagging sleeping animals during the day with thorns were probably highly uncertain enterprises (Monson et al., 2003), giving that flying foxes have emminent visual and auditory awareness. The apparent pulse of ALS-PDC during the $20^{\text {th }}$ century has been suggested to be due to an increase in the consumption of flying foxes in the aftermath of the military occupation of Guam, when the Chamorros gained access to firearms and disposable incomes as Guam was developed as a military base (Monson et al., 2003). Consumption of $P$. mariannus waned, however, as the availability of flying fox became poor. The decline in the population of flying foxes were due, in part, to deforestation, introduction of brown tree snakes in the latter part of the $20^{\text {th }}$ century, and especially to overhunting. The amount of neurotoxin derived from a flying fox dish would eclipse any alternative source of BMAA in the Chamorro diet, particularly among those who ate flying foxes with some frequency.

In another part of the world, in Guadeloupe, the French West Indies, an unexpectedly high percentage of an atypical parkinsonism unresponsive to levodopa has been observed. A small case-control study was performed to investigate the possible relation of this atypical form of parkinsonism with consumption of tropical plants. On the basis of the results, it was hypothesised that chronic exposure to neurotoxic alkaloids in herbal teas (e.g. from Annonaceae species) could be an important aetiological factor because these compounds induce parkinsonism in animals (Caparros-Lefebvre et al., 1999).

\subsection{Conclusions on cycad and BMAA exposure and degenerative neurological disease}

A review of information available in published literature supports a true connection between cycad esxposure and the development of ALS-PDC 
on the island of Guam. However, it is not clear which the compound(s) in the cycad is that is responsible for the development of neurological disease. One candidate today is the non protein amino acid $\beta$-methylaminoalanine.

From the information given in published literature it has neither been possible to compare the potential exposure to cycads or BMAA in the different high-incidence populations of ALS-PDC in the Western Pacific, nor to assess the bioavailability of BMAA from the different exposure routes. It would be of interest to obtain information on the actual incidences of disease in the other high risk areas in Kii Peninsula and western New Guinea. Due to lack of information it is difficult to comment on BMAA as a proposed common denominator connecting these three populations with a of high incidence of neurological disease. We have found no information on the use of cycads in other parts of the world, and, therefore, been unable to correlate the incidence of ALS-PDC in other populations with cycad or BMAA exposure.

The time factor between exposure to cycad products and manifestation of neurodegenerative disease is an issue that need further clarification. Cox and co-workers have suggested a mechanism that allows a slow release of toxins from a depot. It has been suggested that this depot is BMAA bound in different matrices. Most notably it has been proposed that BMAA is incorporated into proteins or small peptides, or alternatively strongly linked to these macromolecules. However, no mechanism for such an interaction is known. It would be of great importance to find an answer to the questions of whether and how BMAA could be bound in tissue, in order to resolve the theory of a slow-acting neurotoxin released from a tissue-reservoir, and to conclude on the discussion of a possible biomagnification of the amino acid. 


\section{Case reports on animals, toxicological studies on cycad materials and preparations}

Because of its content of powerful emetic, teratogenic, and hepatotoxic agents, the raw female gametophyte tissue of cycads can not be consumed. The consumption of only a few unprocessed cycad seeds at one sitting has been reported to result in acute gastrointestinal disturbances (Kurland, 1988). In addition, there are effects of chronic exposure to the raw cycad seeds. As a consequence, the Chamorro people have learnt that the seeds have to be detoxified before they are used.

It is well known that livestock (cattle, sheep, horses and pigs) grazing on cycads may develop paralysis. It is not known which toxic substance is responsible for the condition, but BMAA has been suggested by some investigators.

As the cycads contain several toxicants, it may be difficult to allocate the toxicity profile after cycad product consumption to a single compound. Over the years different natural toxicants of the cycad have been in focus of the scientific interest to explain the biological activity of these plants. Initially, the carcinogenic compound cycacin (methylazoxymethanol $\beta$-D-glycoside) and its active aglycone methylazoxymethanol (MAM) attracted most interest among toxicologists and epidemiologists, before BMAA became of interest. In this report we present available information to explore whether it is possible to allocate the toxicity to BMAA, thus we also present information from studies intended to study the effect of cycacin in cycad products. As BMAA was initially isolated and described in 1967, all studies published before this date only focused on cycacin and its active metabolites.

A table summarizing comments on the studies performed with cycad preparations is found in Annex 2 of this report.

\subsection{Case reports on animals}

Albrektsen et al. (1998) collected records from the National Animal Poison Control Center in the United States 1987-1997, in order to report clinical end epidemiologic information on cycad poisoning in dogs. Sixty incidents where dogs showed clinical signs were evaluated and eight were categorised as possible toxicoses, 36 as suspected toxicosis and 16 as toxicoses. Cycas revoluta was the most commonly involved cycad 
species, followed by C. circinalis and Zamia floridana. Most dogs (39\%) ingested seeds, but all parts of the plant, including roots and leaves induced signs of toxicoses. Although the amount of cycad material ingested was mostly unknown, as few as $1-2$ seeds caused severe intoxications, influenced serum levels of liver enzymes and resulted in death. Ninetyfive percent of the dogs developed liver- or gastrointestinal problems. More then half of the affected dogs showed one or more neurological sign, including weakness, ataxia, depression, preoprioceptive deficits, coma or seizures. It is not known whether the neurologic signs observed were secondary to liver damage or the result of neurotoxins. Onset and duration of clinical signs ranged from 15 minutes to 3 days and from 24 hours to 9 days, respectively. Recovery rate was $68 \%$.

It is well known that livestock (cattle, sheep, horses, and pigs) grazing on cycads (Cycas, Bowenia, Lepidozamia, and Macrozamia) may develop a hind-limb paralysis (Hooper, 1978, 1983). The condition is characterized by animals swaying their hind quarters, failing to fix their joints properly, thereby over-dancing on the hind limbs. Although it is far from sure that BMAA is the toxic agent responsible for this conditon, Duncan (1991) analysed leaves of Australiasian cycads (growing in the Fairchild Tropical Gardens, Miami, USA) for free BMAA. The result of these analyses is presented in Table 4 of Chapter 6 . It turned out that free BMAA is found in cycads from all species studied but levels were highest in some of the species belonging to the genus Cycas.

Using the data in Table 4 on the levels of free BMAA in leaf tissue of various cycads to estimate the dietary exposure in reported cases of cycad poisoning where feed intake was known, there are indications that cycad poisoning may occur at very low levels of free BMAA (corresponding to $0.56 \mathrm{mg} / \mathrm{kg}$ body weight). Because BMAA toxicity in primates requires administration of daily doses of more than $100 \mathrm{mg} / \mathrm{kg}$ over a period of 3 months (see Spencer et al, 1987a, Section 4.4), Duncan (1991) concluded that if BMAA is the agent responsible for Zamia poisoning (namned after the cycad species giving rise to the intoxication) in cattle and sheep, these animals must be remarkably sensitive to the neurotoxic effects of the relatively small amounts of BMAA found in leaves. If the observed levels of free BMAA are good markers for the total level of BMAA, the chemical analytical data shown in Table 4 indicate that high doses of BMAA are unlikely to arise from consumption of cycads other than those of the genus Cycas. Therefore, it might be another toxic principle that is responsible for livestock poisoning. 


\subsection{Studies on primates fed preparations from Cycas circinalis}

A few studies where primates have been fed cycad products have been published. In general, the aim of these studies was to investigare the carcinogen cycasine occuring in the plant.

The first of these studies was a pilot study with four rhesus monkeys (Dastur, 1964). The study is scantily reported, and only gives superficial information on animal care and housing, as well as on clinical and physical investigations and histology. One monkey served as control, while three monkeys were fed cycad flour prepared from seeds of Cycas circinalis. Two types of cycad flour was used, one type being prepared for food use by residents of Guam, the other by the investigators in their own laboratory. The latter flour was prepared from kernels that during 8 days were washed in three changes of water, and then dried and powdered. Analyses for the carcinogen cycacin revealed no cycasin in the flour from Guam, whereas the flour prepared by the investigators contained $10 \mathrm{~g} / \mathrm{kg}$ flour. After being stored for one year, the flour was used to bake "pancakes" that were served to the monkeys. The actual amount of cycad flour ingested was estimated from the pancake recipe and the weight of the pancakes consumed.

The first treated monkey, 4.5 years old at the beginning of the experiment, received $2 \mathrm{~g}$ Guam-produced cycad flour for 8 days and $4 \mathrm{~g}$ laboratory-prepared flour for 18 days. As the monkey obviously only consumed a fraction of the daily ration, it had after 26 days of feeding ingested in total $15 \mathrm{~g}$ cycad flour. Assuming the monkey ingested the same amount of cycad flour every day, $12.2 \mathrm{~g}$ of the ingested flour would have been of the kind that contained $1 \mathrm{~g}$ cycasin/100g. The monkey would then have ingested $0.12 \mathrm{~g}$ cycasin during the course of treatment. This monkey was anorectic at the end of the cycad flour feeding and showed prostration and hemorrhages in the anterior aspect of both arms. On day 33, the monkey developed a habit of pressing on the right eye when focusing, as if to compensate for diplopia, a habit which continued for 16 months. Between month 4-7 the monkey exhibited weight loss, loss of hair, and mild microcytic anemia. The hair had grown out again after 8 months. Wedge liver biopsy was essentially normal after one year.

The second monkey was 9 years old and weighed $4.14 \mathrm{~kg}$ at the beginning of the experiment. This monkey received $2 \mathrm{~g}$ Guam-produced flour for 8 months and $4 \mathrm{~g}$ of laboratory-produced flour for 6 months. This monkey consumed $254 \mathrm{~g}$ cycad flour during 14 months of feeding. Making the same assumptions as for monkey n:o 1, monkey n:o 2 would have ingested $1.52 \mathrm{~g}$ cycasin during the last 6 months of the study. The monkey suffered a mild hypochromic anemia, but no other toxic effects were seen. Wedge liver biopsy after 9 months on the cycad diet showed 
mild bile duct proliferation with a few inflammatory foci. Deltoid biopsy was normal after 10.5 months.

The third monkey was half a year old and weighed $2.90 \mathrm{~kg}$ at the beginning of the study. This monkey received $1 \mathrm{~g}$ Guam-produced flour for 8 months and $2 \mathrm{~g}$ laboratory-produced flour for 2 months. After a feeding period of 10 months the monkey had ingested in total $95 \mathrm{~g}$ cycad flour. Making the same assumptions as for monkeys 1 and 2, this monkey would have ingested about $0.29 \mathrm{~g}$ cycasin during the last 2 months of feeding. This monkey had leg weakness for 2 weeks after 3.5 months of feeding. After 7 months weight loss, loss of hair and anorexia were noted. The monkey died after 10 months of feeding following 1.5 month of progressive emaciation and facial edema, severe weakness and wasting of muscle in one arm, anemia and elevated levels of serum glutamicoxalacetic transaminase and serum glutamic-pyruvic transaminase (SGOT and -SGPT, nowadays mainly referred to as ASAT, aspartate aminotransferase, and ALAT, alanine aminotransferase). Autopsy showed skin atrophy with collagen degeneration, athrophy of muscles of limbs and trunk, degeneration of anterior horn cells, degeneration and partial loss of pyramidal neurons of motor cortex with some glial reaction and mild subcapsular rarefaction (loss of density and weight but not of volume) and rare fibrous scarring of the liver.

The fourth monkey was a control receiving no cycad flour. Biopsies of liver, skin and muscle from this monkey were normal after 10.5 months of the study (Dastur, 1964). The author concluded that rhesus monkeys appeared to be suitable for studying the toxicity of cycads.

If an attempt is made to hypothesise a possible BMAA intake by the monkeys in this study, it is necessary to chose a figure for BMAAcontent in cycad flour. Using the highest figure reported (Duncan et al, 1991), $146 \mathrm{mg}$ BMAA/kg flour, as a worst case scenario, the total amount BMAA ingested by monkeys 1, 2 and 3 would have been $2.2 \mathrm{mg}, 237 \mathrm{mg}$ and $13.9 \mathrm{mg}$, respectively. The daily doses would have been $0.1 \mathrm{mg}, 0.4$ $\mathrm{mg}$ and $0.05 \mathrm{mg}$ BMAA respectively. These hypothetical BMAA doses from cycad fluor are several hundred times lower than the doses of synthesized BMAA that Spencer et al (1987a) gave to monkeys in an experiment that is cited in chapter 4 . Taking all weaknesses into account, it is not possible to determine any potential effects arising from ingestion of BMAA on the basis of this pilot study.

With the objective to describe the different pathological changes occurring in various organs after consumption of different cycad flour preparations, Dastur and Palekar (1974) followed-up on their pilot study with a larger study on monkeys. This study was not much better reported.

Sixteen monkeys were used, of which three were controls. Species, age and weight of the monkeys were not given. They were fed three different cycad preparations; washed, unwashed or boiled cycad flour containing $0.001,1.35$ and $1.46 \%(\mathrm{w} / \mathrm{w})$ cycasin, respectively. The flour was 
incorporated into "chapatis" or banana pulp, or was administered in the form of sweet balls, at a level of 1-2 g/day. No information was given on the actual length of feeding the experimental diet to the monkeys, or the amount of cycad flour ingested. It is indicated that monkeys were fed cycad for at least two years. The total dose of cycad flour ingested by the monkeys ranged from 89 to $1454 \mathrm{~g}$. At least three animals died during the experiment, but the times of deaths was not stated.

Of the five monkeys receiving washed cycad flour, one showed signs of acute hemorrhagic disorder with transient evidence of hepatic insufficiency but recovered completely and had a long survival. Another animal in this group suffered from slowly progressing hepatic toxicity, manifesting towards the end of the second year of cycad feeding. A third animal in this group was generally malnourished and died of infection.

Unwashed cycad flour was given to five monkeys. Two of these showed indications of hepatotoxicity in the form of a reversed serum albumin-globulin ratio, elevated transaminase levels and jaundice. One of the animals died in hepatic coma.

Three animals received boiled cycad flour. Two of these showed the same signs of liver toxicity as the ones receiving unwashed cycad flour, and one of these animals died of hepatic coma.

The monkeys fed various cycad preparations showed frequent degenerative changes in the liver amounting to cirrhosis, and less frequent but impressive regenerative changes in the liver. Other organs appeared unaffected clinically and pathologically by the diet, according to the authors (Dastur and Pallekar, 1974). However, it is not stated what pathological or histological examinations were carried out. The study is very poorly described, and due to lack of information the study can not be considered relevant.

Garruto et al. (1988a and b) made an attempt to develop a primate model of ALS/PDC based on chronic interaction between low calcium intake and high intake of toxic metals. In this connection they also tested the effect of long-term cycad feeding. Cynomolgus monkeys (Macaca fascicularis) were used in the study. One control monkey was maintained on a normal monkey diet. Two monkeys were given a specially formulated low-calcium diet. Another two monkeys were maintained on the low calcium diet with daily supplements of $150 \mathrm{mg}$ aluminium, $50 \mathrm{mg}$ manganese, whereas yet another two monkeys received the same diet supplemented with $2 \mathrm{~g}$ unprocessed cycad flour prepared from unwashed seeds of C. circinalis. The study was continued for 4 years. No clinical neurological signs developed within 41-43 months. Monkeys in all groups, except the control group, developed motorneuron lesions consisting of neurofibrillary tangles, axonal spheroids chromatolysis, aberrant perikaryal accumulation of phosphorylated neurofilament and basophilic and hyaline-like inclusions in the spinal cord, brainstem, substantia nigra and cerebrum. The lesions were most severe in the monkeys receiving 
supplementation of $\mathrm{Al}$ and $\mathrm{Mn}$. The fact that the lesions occurred also in monkeys not fed cycad indicated that the lesions were not a consequence of ingestion of cycad (Garruto et al, $1988 \mathrm{a}$ and b). The study was designed to search for an experimental model for metal-induced neurofibrillary degeneration. It is not possible to draw conclusions on BMAA toxicity from this study.

\subsection{Studies on rodents fed preparations from Cycas circinalis}

In an investigation aiming at studying the effect of cycad intake on ALS and carcinogenicity, male and female Osborn-Medel rats were fed diets containing cycad flour for nearly two years (Yang et al., 1966). Three groups of 15 rats each (equal numbers of males and females) were given diets containing $1.5 \%, 5.0 \%$ or $10 \%$ cycad flour from Cycas circinalis. Another 15 rats served as controls. The flour had been prepared and processed by natives in Guam and was obtained from local markets representing different areas of the island. Rats were 4 weeks old at the start of the experiment. They were housed individually and given food and water ad libitum. To evaluate the neuromuscular condition of the animals, males of all groups were placed in activity cages, given one week of adaption whereafter spontaneous activity was recorded during weeks 7 and 8 of the feeding experiment. The average voluntary activity was not significantly different between treatment groups.

Eleven of the dosed animals, spread evenly between dosage groups, died or were killed because of their moribund condition. Causes of death were pulmonary infections (4 animals), reticulum cell sarcoma (1 animal), adenoma of the pancreas ( 1 animal), tumours of the pituitary gland ( 3 animals), and in two animals the cause of death could not be determined. All 15 control rats survived to the end of the study. There were no significant differences in body weight between the groups. However a greater depression in body weight was observed in all experimental groups compared to control group when there was a sudden drop of temperature in the animal quarters. When the temperature was corrected the animals regained their lost weight in two months time. Complete autopsies, including histopathology of all internal organs and endocrine glands, were performed on all rats. Several adenomas or foci of adenomatous hyperplasia were found in endocrine organs. The numbers of animals in each group that did not reveal any endocrine lesions were 4 in the control group, 6 in the $1.5 \%$ cycad flour group, 7 in the 5\% group, and 5 in the $10 \%$ cycad flour group. The authors drew the conclusion that the tumours and hyperplastic processes found at autopsy were related to advanced age of the animals rather than to ingestion of processed cycad flour. They also concluded that the local customs of preparing cycad flour removes poten- 
tial toxicants and carcinogens effectively, if properly executed (Yang et al, 1966).

There is no mention of pathological lesions in the central or periferal nervous system, or of neurological or motor disturbances of any of the animals in this study. However, it is not stated how often and to what extent the animals were observed and, nor to what extent the histological examinations were carried out. One female rat that died during the study was autolyzed to an extent that detailed histology could not be performed, and cause of death could not be determined. This information may implicate that rats were not adequately observed on a regular daily basis, and that there may have been periods of time where they were not properly attended to. In addition, the incident with a drop of temperature in the animal quarters, that obviously was of a severness and duration that resulted in weight loss or reduced weight gain in animals, implicates that a proper surveillance of the animals may not have been carried out. The study does not give any input to the potential toxicity of BMAA.

Another feeding experiment on rats was performed with the object of determining the influence of age, strain and cycad diet on growth. Several experiments were included in this study but many important details of the set-up of the experiments are lacking in description. Animals were fed diets containing 0, 2 or 4\% cycad flour prepared from seeds of Cycas circinalis for periods from 5 days to 2 weeks (Campbell et al., 1966). The parameters studied were mainly weight gain/loss. Soaking and incubating flour in water, heating and storage, reduced cycad toxicity, probably due to enzymatic and chemical degradation of cycasin. Younger rats appeared to be more severly affected by cycad toxicity than older rats, while rat strain, sex and origin did not influence cycad susceptibility. Rats that had been fed $2 \%$ cycad in the diet for 2 weeks died with symptoms of cycad toxicity even after the cycad flour feeding had been discontinued (Campbell et al, 1966). Included in this study is an experiment where a heifer was fed $2 \%$ unwashed cycad flour in its feed. After 43 days the heifer developed neurological disturbances and was slaughtered. The liver was dried and ground and incorporated in feed that was given to weanling rats. The amount of ground liver incorporated in the rat fodder was 2, 5 or $10 \%$. Weight gains of the rats receiving the ground liver did not differ from control rats (Campbell et al, 1966). It is not possible to draw any conclusions on either cycad or BMAA toxicity from this paper. It is interesting, however, to note the divergent approaches that have been executed in attempts to study the properties of cycad toxicity.

In the most recent study on cycad toxicity, a group of seven 6-7 month old male CD-1 mice was fed $0.5 \mathrm{~g}$ washed cycad flour daily for up to 30 days (Wilson et al, 2002). Control mice were given pellets of identical weight and similar nutritient content from commercial grade processed white flour. The washed cycad flour contained $0.144 \mathrm{mg} \mathrm{BOAA} / \mathrm{kg}$ and $0.003 \mathrm{mg}$ BMAA $/ \mathrm{kg}$. Methylazoxymethanol (MAM) and cycasin 
could not be detected at a detection limit of $0.08 \mu \mathrm{g} / \mathrm{kg}$. Washed flour samples were analysed by HPLC-MS after extraction with distilled water and filtration. No further details on the analytical method was given in this report, but Khabazian et al (2002) give more detailed information. To our knowledge this is one of very few reports that state that BOAA is present in cycad flour from Cycas circinalis, and this might be of significance when considering the interpretation of the study. The animals consumed in total between 8.9 and $11.2 \mathrm{~g}$ cycad flour per mouse, with an average consumption of $10.1 \mathrm{~g} /$ mouse. This equals between 0.20 and 0.30 $\mathrm{mg}$ flour $/ \mathrm{kg}$ body weight with the average being $0.24 \mathrm{mg} / \mathrm{kg}$ for 26 days. There were no significant differences in body weight between control and dosed animals.

In the Wilson study (2002) the rotarod test, latency to fall from a rotating rod, was used to evaluate motor coordination and symptoms of possible motor neuron degeneration. Gait and locomotion were measured by the de Medinacelli pawprint test. Leg extension reflex test was used as a measure of motor neuron dysfunction. To evaluate nonspatial reference memory the water maze was used, and the radial arm maze was used to evaluate working and reference spatial memory. In mice fed cycad flour motor deficits began to appear after 2 days of feeding, and became significant by day 7 . Hind leg extension deficits gradually increased in cycad-fed mice, reaching significance by day 14 . These findings were accompanied by a noticeable tremor of the hind limbs, which increased even after the cessation of cycad feeding on day 30. Cycad-exposed mice were able to remain on the rotarod for the same length of time as control mice, but adopted a hunched position, leaning against side barriers, that was not noted for control mice. In the water-maze testing cycad-fed mice obtained significantly longer latencies than control animals by day 10 . These differences diminished with additional trials. Data for radial-arm maze demonstrate that control mice had significantly lower numbers of entry errors than cycad-fed mice on various test days.

Histological assessment of apoptosis in sections of brain and spinal cord was made using TUNEL (Terminal deoxynucleotidyl Transferase Biotin-dUTP Nick End Labeling) staining and detection of caspase activity (Wilson et al, 2002). All cycad-fed mice exhibited similar TUNEL positive staining in the same CNS regions. Such labelling was not found in the tissues of any of the control mice. Caspase- 3 antibody labelling for apoptosis was positive in regions of cycad-fed mouse brain but were not seen in control mice.

Abnormal tau expression is a key characteristic of neurofibrillary tangles (NFT) occurring in Alzheimers disease and in ALS-PDC. Tau-2 antibody labelling showed labelled cells in various hippocampal fields, substantia nigra and regions of cortex of cycad-fed mice. Controls also showed tau-positive cell bodies in hippocampus and cortex, but less than half as frequent as did cycad-fed mice. Motor neuron counts of cervical 
spinal cord showed significantly decreased number of motor neurons in cycad-fed animal compared to controls (Wilson et al, 2002). The only other organ that was examined histologically was the liver. The two groups of mice showed no morphological difference or any difference in staining. Also no significant apoptosis or morphological changes were found in the livers of cycad-fed mice (Wilson et al, 2002).

Wilson et al (2002) drew the conclusion that a neurotoxin, or a toxic metabolite, not previously identified in cycad, is able to gain access to the central nervous system resulting in neurodegeneration of specific neural populations and in motor and cognitive dysfunctions.

\subsection{Rodent studies on other cycad species than C. circinalis}

Rodent feeding studies have also been performed with other cycads than C. circinalis. Gobé (1994). This study presents reappraisal of archived tissue samples from a study originally publicated in 1985 by Gobé and Pound. This original publication could not be obtained. Thus, it is possible that information that was not included in the publication by Gobé (1994) may be found in the original report of the study. Freeze-dried seeds of Lepidozamia peroffskyana were mixed with a standard animal diet to contribute with $5,10,15$ or $30 \%$ of the diet. These diets were fed ad libitum together with water to groups of adult Quackenbush male mice, the number of animals per treatment was not stated. Control groups were fed standard rodent diet. During re-appraisal of archived liver, gut, kidney and brain tissue, apoptosis was identified in histological sections of brain and gut tissue. Control tissues appeared normal. It was not stated how the archived tissues had been stored. Most toxic effects were seen in tissues from mice fed the two highest doses of cycad after 2 and 4 days of feeding. In addition, liver tissue was affected with necrotic patches. There was a correlation between concentrations of cycad in the diet, length of exposure and liver lesions. Apoptotic lesions initially described as degenerative changes in the gut, were during reappraisal proven to be apoptotic lesions. On re-examination, brain tissue appeared to have greatly increased apoptosis in animals that received cycad, but the cell type undergoing apoptosis was not identified. The authors speculate that an understanding of apoptotic control mechanisms might shed some light on the relationship between consumption of cycad products and the degerative diseases ALS-PDC (Gobé, 1994).

In vivo toxicological studies on cycad preparations are summarised in Annex 2. 


\subsection{Conclusions on case reports and toxicological studies performed with cycad preparations.}

The cycad tree and its seeds contain several toxicants (e.g. cycasin, methylazoxymethanol [MAM] $\beta$-D-glycoside, BMAA) and it is difficult to allocate effects of cycad ingestion to any single compound. Also, due to the use of different experimental approaches in the feeding studies that have been performed with cycad preparations it is difficult to aquire a comprehensive picture of cycad or BMAA toxicity. This difficulty is reinforced by several of the early studies being deficient in study design, quality assurance and reporting of experimental parameters and data.

It is clear that ingestion of raw, unprocessed cycad may induce both acute and chronic toxicological effects. Processed cycad preparations, where the levels of known toxicants have been reduced to very low or undetectable levels may also induce toxic effects.

The authors of one study of good quality (Wilson et al, 2002) concluded that an as yet undiscovered toxin must be responsible for the neurodegenerative disease in Guam, since their study showed neurological lesions to be induced in mice fed cycad flour even when the amounts of cycasin, MAM, BOAA and BMAA had been reduced to very low or undetectable levels in the flour. 


\section{BMAA as a natural toxicant in cycads and studies on BMAA toxicity}

As mentioned in the previous chapters, cycads contain several bioactive compounds that may be responsible alone or in combination for the adverse effects reported in humans and animals consuming cycad plant tissues or products derived thereof. As outlined in chapter 2, cycacin and its active metabolite methylazoxymethanol, was early deleted from the list of candidates responsible for the neurodegenerative disease on Guam. The search for other toxins than cycasin in cycads was succesfull 1967, when BMAA was isolated and described (Vega and Bell, 1967). The neurotoxic activity of this compound was confirmed by administering BMAA to chickens and young rats. In this study only the L-isomer produced toxic effects (Vega et al, 1968). As a consequence of BMAA being identified in cycad seeds, the hypothesis that this compound was responsible for the high incidence of ALS-PDC in Guam was presented, and this hypothesis stimulated further studies on BMAA. Annex 2 summarizes the in vivo toxicity studies conducted with BMAA.

\subsection{Biosynthesis}

Not much is known about the genes and enzymes involved in the biosynthesis of BMAA. As mentioned in the introduction to this report, Banack and Cox (2003b) have proposed that cyanobacteria in coralloid roots of cycads produce BMAA and may be responsible for the occurence of BMAA in cycads, but without presenting a pathway for the biosynthesis. However, a pathway for BMAA biosynthesis in cycads is proposed on theoretical grounds in a publication by Brenner et al. (2003a). It is assumed that BMAA is produced by the cycad, and not by organisms in symbiosis with the plant. Candidate genes for enzymes involved in the pathway were identified from a library from cycad leaves. As the BMAA structure is fairly similar to other $\beta$-substituted alanines, it was proposed that phosphoserine, cysteine, o-acetylserine or cyanoalanine may be precursors for BMAA biosynthesis. Based on this assumption, the pathway for BMAA biosynthesis illustrated in Figure 2 was suggested. 


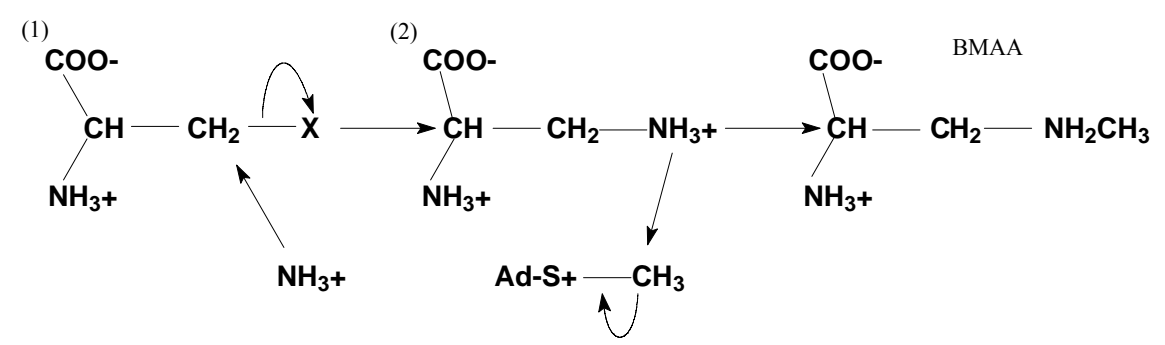

Fig. 2 Proposed pathway for BMAA biosynthesis in cycads. (1) Ammounium is transfered to a $\beta$-substituted alanine ( $X=$ substituent giving rise to phosphoserine, cysteine, $o$ acetylserine or cyanoalanine) to form the intermediate (2). The protonated amino group on the intermediate attracts a methylgroup from S-adenosylmethionine $\left(\mathrm{Ad}-\mathrm{S}-\mathrm{CH}_{3}\right)$ via a methyltransferase, thereby forming BMAA. (Figure adapted from Brenner et al., 2003a).

Although it is not known whether the proposed reactions take place, an expressed sequence tag (EST) library from cycad leaves contains candidate genes for all the enzymes predicted for the suggested biosynthetic route, including two ESTs which encode cysteine synthases (Brenner et al., 2003a). The library also contains two potential methyltransferases (caffeic acid O-methyltransferase II and caffeoyl- $\mathrm{CoA}_{3}-\mathrm{O}$-methyltransferase) that may catalyse the second biosynthetic step.

The methyl donor for the second step may be S-adenosyl-methionine (SAdM). However, depletion of SAdM would require an enzymatic machinery regenerating SAdM. A number of cycad ESTs may be implicated, including adenosylhomocysteinase, $S$-adenosylmethionine synthetase and homocystein methyltransferase (Brenner et al., 2003a).

\subsection{Effects in plants - a possible mechanism of action}

What is the role of BMAA in cycads? Does it have a role in the protection against predators, or a physiological role, possibly as an endogenous signalling compound (Brenner, 2003b). BMAA is an agonist of glutamate receptors. Glutamate receptor genes have been purified from cycads in BMAA-containing tissue. In Arabidopsis thaliana, the putative glutamate receptor 1.1(AtGLR1.1) functions as a regulator of carbon and nitrogen metabolism (Kang and Turano, 2003).

Glutamate (Glu) is one of the predominant neurotransmitters in the mammalian brain. As a neurotransmitter, it activates Glu receptors at post-synaptic membranes, which are involved in sensing environmental cues and in memory function (Medina and Isquierdo, 1995; Tsien et al., 1996). Improper ionotropic Glu receptor (iGluR) function has been implemented in a variety of human diseases, including Alzheimers and Parkinsons dementia (Ikonomidou and Turski, 1996; Ikonomidou et al 1996; Forsythe and Barnes-Davies, 1997). One subgroup of Glu receptors is comprised of the iGluRs (iGluRs are ligand-gated ion channels that are the predominant neuroreceptors in the mammalian brain) that convey 
rapid synaptic transmission. iGluRs are pharmacologically classified into subgroups based on agonist response. The two main iGluR subfamilies in animals are N-methyl-D-Asp (NDMA) and $\alpha$-amino-3-hydroxy-5methylisoxazole-4-propionic acid (AMPA/kainite (KA) (non-NDMA) activated iGluRs (Ikonomidou and Turski, 1996; Forsythe and BarnesDavies, 1997; Lam et al 1998a).

In plants Glu acts as a signalling molecule altering the expression of genes encoding enzymes involved in amino acid metabolism (Lam et al., 1994, 1998b; Oliveira and Coruzzi, 1999). However, the mechanism is poorly understood. Genes with a high sequence similarity to animal iGluRs have been identified in Arabidopsis. Arabidopsis GluRs have all the signature features of animal iGLRs, including a plasma membrane signalling peptide, two putative ligand-binding domains, and a "threeplus-one" transmembrane region (Lam et al., 1998a; Chiu et al., 1999). To assess the function of putative Glu receptor genes in plants, Arabidopsis seedlings were treated with the iGluR antagonist 6, 7dinitropuinoxaline2, $3(1 \mathrm{H}, 4 \mathrm{H})$ dione (DNQX), known to block AMPA/KA iGLRs in animals (Muller et al, 1988). It was shown that DNQX inhibits two key aspects of seedling photomorphogenesis in Arabidopsis: light-induced hypocotyls shortening and light-induced greening (Lam et al., 1998a). To further explore the targets of AtGLR function in plants, Brenner et al. (2000) tested whether other compounds known to block iGluR function in animals could also block aspects of Arabidopsis growth and development. Because BMAA is a natural plant product that blocks iGluR function in animals, these investigators tested whether it would have any effect on plant GluRs using Arabidopsis as a model. It was shown that BMAA (at low concentrations) promotes hypocotyls elongation and inhibition of cotyledon opening when applied to light-grown Arabidopsis seedlings. L-Glu, and to some extent Lglutamin, but not L-asparatate reversed the effect of BMAA on early morphogenesis.

A study by Brenner et al. (2000) showed that BMAA lightdependently induces hypocotyl elongation in Arabidopsis. Because BMAA exerts its effects at low concentrations $(20-50 \mu \mathrm{M})$ this suggests that BMAA could act as a signalling compound in plants. Phylogenetic studies on glutamate receptors in plants and animals suggests that iGluRs are derived from a primitive signalling mechanism that may have existed before plants and animals diverged. This suggests the possibility that iGluR agonists, such as BMAA, made by plants may serve not only as herbivore deterrents but may also act as signalling molecules affecting developmental processes in plants. Brenner and co-workers postulated that BMAA may also alter light signalling in cycads, and questioned whether BMAA may even be present at low levels in other species of higher plants (Brenner et al., 2000). 


\subsection{Toxicokinetics}

The survival of the hypothesis stating cycad toxins being theacause for the high incidences of neurological disease in three foci in the Pacific, is related to the human use of cycads in all three geographical areas. In one of these, in western New Guinea, the only reported use of cycads is as topical application of cycad poultices. Duncan and co-workers theoretically asessed the significance of this route of administration, focusing on the levels of BMAA that can be reached in the circulation. Based on a known BMAA content in cycad seeds and assuming a daily use of freshly prepared poultice for one month, Duncan et al. (1991) calculated the worst-case daily dose to be $0.36 \mathrm{mg} / \mathrm{kg}$ bodyweight and the accumulated dose to be $10.8 \mathrm{mg} / \mathrm{kg} /$ month. (Duncan et al, 1991).

Without knowledge of how the poultices are prepared and applied, it is impossible to ascertain the validity of this theoretical calculation. thus, it is necessary to have information on the formulation of the poultices used, in order to be able to assess whether the percutaneous absorbtion of the water-soluble amino acid BMAA would be facilitated by the poultice. The application of the poultice on open wounds may facilitate absorption, but it is not stated for what conditions the poultices are used and not whether they are applied on intact or injured skin. As of today we have not found any experimental data on percutanous absorption of BMAA. As absorption through topical poultices constitutes the basis for BMAA exposure from cycads as a culprit for the disease in Western New Guinea, it is of interest to to obtain information about bioavailability by this route.

The oral bioavailability, pharmacokinetics and blood-brain barrier permeability of L-BMAA were studied in adult male Sprague-Dawley rats (260-380 g) after intervenous injection of a single dose of 25, 100 or 400 mg BMAA hydrochloride per kg body weight (Duncan et al., 1991). No specific behavioural tests were performed on the rats. However, no alterations in behaviour were noted in animals receiving acute doses of BMAA, except in one animal given $400 \mathrm{mg} / \mathrm{kg}$, which demonstrated spasms and arched tail. Plasma samples, urine and faeces were collected from 0.25 minutes to 3 days after dosing, and subsequently analysed for BMAA by GC/MS (Duncan et al., 1989). In the chemical analysis $\left[{ }^{2} \mathrm{H}_{3}\right]-$ BMAA was used as internal standard (tissue samples were added internal standard and $1 \mathrm{M} \mathrm{HCl}$ before disruption and extraction).

BMAA was quickly cleared from the bloodstream and plasma concentration fell to less than $5 \%$ of the initial value within 30 minutes after the administration. The compound was concentrated in peripheral tissue and reached concentrations $(20-500 \mathrm{mg} / \mathrm{kg})$ exceeding those in plasma by a factor 3-50. The accumulation in the brain was more limited, with concentrations rising to $4-10 \mathrm{mg} / \mathrm{kg}$ within 2 hours of injection. Initially the highest concentrations in the CNS were found in pons, thalamus and cerebellum, and the lowest in the spinal cord, cerebral cortex and cere- 
brospinal fluid (CSF). Less than $0.08 \%$ of the injected dose was estimated to be present in brain $2 \mathrm{~h}$ after injection. Plasma and brain concentrations were directly related to dose. After the $100 \mathrm{mg} / \mathrm{kg}$ dose, the half-lives of BMAA in plasma and tissues were found to be at a comparable level $\left(\mathrm{t}_{1 / 2}=11-17 \mathrm{~h}\right)$.

Oral bioavailability of BMAA hydrochloride was determined from data on 3 rats administered $100 \mathrm{mg}$ BMAA $/ \mathrm{kg}$ body weight by gastric intubation by measuring the plasma concentrations for $8 \mathrm{~h}$. Absorption was rapid and fairly complete (around $80 \%$ ) with plasma concentration reaching a maximum 5 minutes after dosing. Eight hours after BMAA administration, the cortical brain concentration of BMAA averaged $3.6 \pm 0.7 \mathrm{mg} / \mathrm{kg}$, which was $82 \%$ of that in the intravenously injected animals $(4.4 \pm 1.2 \mathrm{mg} / \mathrm{kg})$. Extrapolation of the curves for the plasma concentration of BMAA, indicated that the absolute bioavailability of BMAA may exceed $93 \%$.

Rats that were infused BMAA hydrochloride subcutaneously at a constant rate of $100 \mathrm{mg} / \mathrm{kg}$ body weight/day for 7 or 14 days, respectively, showed similar tissue and fluid concentrations of BMAA at these two time points. Regional brain levels ranged from 10 to $30 \mathrm{mg} / \mathrm{kg}$ and were 2-3 times higher than the maximum values reached after a single intravenous injection. The BMAA concentration was significantly higher in thalamus and hippocampus than in cortex or CSF, in agreement with the findings after a single intravenous injection of the compound. Mean plasma concentration was $9.5 \mu \mathrm{g} / \mathrm{ml}$. Brain-to-plasma ratios varied from 1 to 3 and exceeded the CSF-to-plasma ratio 10- to 30-fold, indicating brain cellular accumulation. Chronically treated animals appeared to behave and gain weight similar to control animals.

Studies on uptake of BMAA into the brain showed that the coefficient of transfer of BMAA was less than or equal to values reported for the least permeant natural amino acids, such as glycine, proline and aminoisobutyric acid, and comparable to values for tracers such as sucrose, mannitol and urea, which are used as markers of passive permeability through the blood-brain-barrier. Values for BMAA were 10 to 100 times lower than for more permeant amino acids such as phenylalanine, leucine and lysine which are transported via carrier systems. BMAA was cleared from brain with a $t_{1 / 2}$ of $14-17 \mathrm{~h}$.

When tissue homogenates were incubated in vitro, the concentration of BMAA did not change significantly during 2 hours, which according to Duncan and co-workers indicates a slow rate of BMAA biotransformation.

The results of the studies on oral bioavailability and tissue distribution showed that the low toxicity of oral BMAA found by for example Seawright et al. (1990) and Perry et al. (1989), was unlikely to be due to a poor gastrointestinal absorption of BMAA. It was concluded that BMAA may reach potentially toxic levels in brain after large doses $(>100 \mathrm{mg} / \mathrm{kg})$. 
Such doses were however considered to be orders of magnitude higher than those that would be reached from dietary of medicinal use of cycads. Nevertheless Duncan and co-workers could not dismiss the possibility that BMAA is more toxic in humans than in animalss due to differences in absorption, distribution, metabolism and excretion as well as to differences in cell susceptibility (Duncan et al, 1991).

The bioavailability of L-BMAA has also been studied in 3 cynomologus monkeys (Macaca fasicularis) weighing 2.5, 3.3 and $4.8 \mathrm{~kg}$ (Duncan et al., 1992). Labeled $\left[{ }^{15} \mathrm{~N}\right]-\mathrm{BMAA}$ (2-amino-3- $\left(\left[{ }^{15} \mathrm{~N}\right]-\right.$-methylamino)propanoic acid hydrochloride) (1 $\mathrm{mg} / \mathrm{kg})$ was injected intravenously at the same time that $2 \mathrm{mg} / \mathrm{kg}$ unlabeled BMAA was administered orally by gavage. Both forms were then quantified in a $48 \mathrm{~h}$ urine sample by GC/MS using $\left[{ }^{2} \mathrm{H}_{3}\right]$-BMAA as internal standard (Duncan et al, 1989). One of the monkeys was studied a second time 1 month later when it was given higher doses of BMAA, $9 \mathrm{mg} / \mathrm{kg}$ i.v. and $90 \mathrm{mg} / \mathrm{kg}$ by gavage, to assess oral bioavailability at doses similar to those employed by Spencer et al (1987a) in studies on primate toxicity. After the oral dose $(2 \mathrm{mg}$ BMAA $/ \mathrm{kg}$ ) on average about $80 \%$ was absorbed into the systemic circulation. Two of the three monkeys were fasted before dosing, and one had food and water ad libitum until immediately prior to dosing. There was no difference in bioavailability in these separate cases. After the dose of $90 \mathrm{mg} / \mathrm{kg}$ orally and $9 \mathrm{mg} / \mathrm{kg}$ i.v. given to one monkey three separate urine collections were taken $(0-24 \mathrm{~h}, 24-48 \mathrm{~h}$ and $48-72$ hours). Under theses conditions the oral availability was observed as $100 \%$ (Duncan et al., 1992).

In connection with in vivo studies on neurological effects of BMAA administered by gavage to female CD-1 mice, Perry et al. (1989) studied the uptake of BMAA into brain and liver tissue. BMAA was detectable in brain and liver at $48 \mathrm{~h}$ after dosing (length of study not stated). After a dose of $500 \mathrm{mg}$ BMAA hydrochloride per $\mathrm{kg}$ body weight, the BMAA concentrations in liver varied between 0.1 and $0.3 \mathrm{mM} 24$ hours after dosing. The corresponding levels in the brain were between 0.1 and 0.15 $\mathrm{mM}$ BMAA. At the double dose, $1000 \mathrm{mg} / \mathrm{kg}$ body weight, liver and brain contained 0.2 and $0.5 \mathrm{mM}$ BMAA at 48 hours after dosing, respectively. Only the L-isomer was measured in these studies, as the Disomere was not expected to be re-absorbed in the kidney (Silbernagl, 1988).

It is notable that Duncan et al. (1991) states that the influx of BMAA into the rat brain is comparable to that of tracers used as markers of passive permeability through the blood-brain barrier, and 10-100 times lower than amino acids transported by the large neutral amino acid carrier. Smith et al. (1992), on the other hand, concludes from studies using an in situ brain perfusion technique that BMAA is taken up into the brain by the large neutral amino acid carrier and may be sensitive to the same factors that affect neutral amino acid transport, such as diet, disease and 
age. Possibly these observations might suggest that the affinity to the carrier system is much lower for BMAA than for e.g. other amino acids using the same carrier system such as Phe, Leu, Val, etc.

In vitro studies in neuronal cell culture systems have shown that relatively high concentrations $(>1 \mathrm{mM})$ of BMAA are required to induce toxicity (Spencer et al., 1987a; Duncan, 1991) (see section 4.4 and 4.6). Furthermore, no correlation was found between the BMAA content of extracts of female cycad gametophytes and toxicity to cerebellar granule cells (a primary cell culture of high homogeneity). Hence, if enough BMAA is to reach the central nervous system to result in neuronal destruction, a relatively high blood-brain barrier permeability would be required. However, ALS-PD can develop decades after a limited exposure to cycads. Therefore, a mechanism whereby BMAA can act as a slow toxin - first being accumulated and stored in the CNS, then, after reaching a critical level, giving the biological effect has been searched for. However, Duncan (1991) reports that their studies show that BMAA is rapidly cleared from the circulation, poorly transported into the brain, and that uptake and long-term storage do not take place. Furthermore, preferential uptake of BMAA does not occur into those regions that appear most susceptible to this agent. He concludes that it is unlikely that dietary or medicinal use of cycads would provide sufficient BMAA to produce an acute lesion which, for unknown reasons, would then cause a cascade after 10-20 years of dormancy. He continues, it is also unlikely that a potent neurotoxic metabolite is formed that in itself is capable of manifesting its actions decades after minimal exposure to BMAA.

\subsection{In vivo studies}

In vivo studies on BMAA are summarised in Annex 2.

Vega et al. (1968) investigated the relative toxicities of the L- and Dforms of BMAA. Groups of three male chicks, 7 days post-hatch, were injected intraperitoneally with the L-isomer (3-7 $\mu$ moles/g bw), the same quantity of the D-isomer or with saline solution. Those treated with the Lisomer showed weakness followed by convulsions and head retractions but recovered eventually. Those injected with saline solution or with the D-isomer did not show any of these symptoms. The experiment was repeated with groups of three female Wistar rats who were injected with 6$14 \mu \mathrm{moles} / \mathrm{g}$ bw. Again symptoms in form of dragging gait, weakness and convulsions appeared in animals injected with the L-isomer. No symptoms were noted in the group treated with the D-isomer.

Effects of intraperitoneal injections of DL-BMAA on chicken (3-7 days posthatch), rat and mouse were investigated also by Polsky et al (1972). Chickens were injected with saline (35 birds), $0.20 \mathrm{mg}$ DLBMAA/g bw (1.7 $\mu$ moles)(6 birds), $0.40 \mathrm{mg} / \mathrm{g}$ bw (28 birds) or $0.80 \mathrm{mg} / \mathrm{g}$ 
bw (12 birds). Unmistakable symptoms of toxicity were observed in $0 / 35$, $0 / 6,25 / 28$ and $11 / 12$ birds in the respective dose groups. The rats were dosed with saline ( 6 animals), $0.42 \mathrm{mg}$ DL-BMAA/g bw $(3.6 \mu$ moles $)(3$ animals), $0.84 \mathrm{mg} / \mathrm{g} / \mathrm{bw}$ (12 animals) or $1.68 \mathrm{mg} / \mathrm{g}$ bw (24 animals). Signs of toxicity were observed in $0 / 6,0 / 3,11 / 12$ and 24/24 animals in the respective dose-groups. Similarly, mice were injected with saline (6 animals), $0.84 \mathrm{mg}$ DL-BMAA/g bw (7 animals), $1.68 \mathrm{mg} / \mathrm{g}$ bw (7 animals), $2.52 \mathrm{mg} / \mathrm{g}$ bw (7 animals) or $3.34 \mathrm{mg} / \mathrm{g}$ bw (4 animals). Toxicity was observed in $0 / 6,0 / 7,1 / 7,3 / 7$ and $4 / 4$ animals in the respective dosegroups. Signs of toxicity were generalised extensor convulsions, which began $6-8$ hours post-injection and persisted for $12-18$ hours.

The low concentration of free BMAA in seeds of Cycas circinalis and the relatively high dosages required to produce convulsions in rats, chicks and mice led Polsky et al. (1972) to conclude that his compound is unlikely to be the prime cause of neurological disease in man unless either humans are more sensitive to the compound than the experimental animals, or higher concentrations of the compound occur in the plant in a bound form, or prolonged administration of low concentrations of the amino acid are as effective as large single doses in producing neurological disorders.

The possibility that prolonged administration of low concentrations of BMAA are as effective as large single doses in producing neurological disorders, was investigated by injecting $0.28 \mathrm{mg}(2.4 \mu \mathrm{moles})$ of DLBMAA per gram body weight per day subcutaneously in young SpragueDawley rats for 78 days, a dose one-third of that required to produce generalized extensor convulsions in nearly all rats by a single intraperitoneal injection (Polsky et al., 1972). The prolonged treatment produced no observable neurological change in the intact animal. However, the injections resulted in weakness and reduced growth rate of the animals. The altered growth rate was accompanied by a changed amino acid excretion pattern; more $\beta$-alanine, $\beta$-aminoisobutyric acid, and citrulline and less pipecolic acid was excreted than in control animals. Two unknown ninhydrinpositive compounds not occurring in the controls were observed in the urine of treated animals. Similarly, when newborn rats were treated with $0.14 \mathrm{mg}(1.2 \mu$ moles $)$ BMAA over the first 11 days of life there were no differences observed between treated and control animals (Polsky et al., 1972).

Thirteen one-year-old male macaques (Macaca fascicularis), divided into six groups, were repeatedly administered a freshly prepared aqueous solution of L-BMAA hydrochloride orally for 5 to 13 weeks (Spencer et al., 1987a). Four monkeys received $100 \mathrm{mg}$ BMAA $/ \mathrm{kg}$ bw/day for 12 weeks, another group of four monkeys $250 \mathrm{mg}$ BMAA $/ \mathrm{kg}$ bw/day for 2 weeks and then $125 \mathrm{mg} \mathrm{BMAA} / \mathrm{kg}$ bw/day for 5-7 weeks, resulting in a total exposure period of 7-9 weeks. Two monkeys received $200 \mathrm{mg}$ BMAA/kg bw/day for 6-10 weeks. One of these monkeys was after 6 (or 
10?) weeks of exposure to $200 \mathrm{mg}$ BMAA $/ \mathrm{kg}$ bw/day, given $250-350 \mathrm{mg}$ $\mathrm{BMAA} / \mathrm{kg}$ bw/day for an additional 6 weeks. Three monkeys received 300-315 mg BMAA $/ \mathrm{kg}$ bw/day for 2.5-3 weeks, and one of these monkeys was after the end of this treatment given $200 \mathrm{mg} \mathrm{BMAA} / \mathrm{kg}$ bw/day for an additional 6 weeks. The final group was a control group of 5 monkeys.

The monkeys were inspected daily, clinically examined weekly, evaluated neurophysiologically before and during the treatment period and subjected to neuropathological examination. Treated animals developed corticomotoneuronal dysfunction, parkinsonian features and behavioural anomalies with chromatolytic and degenerative changes of motor neurons in cerebral cortex and spinal cord. Neurological deficits appeared insidiously after 2 to 12 weeks. Signs of motor neuron dysfunction developed symmetrically or asymmetrically in the extremities. Muscle weakness and loss of muscle bulk followed. Two of the four animals receiving $250 \mathrm{mg}$ BMAA $/ \mathrm{kg}$ bw/day for 2 weeks and then $125 \mathrm{mg}$ BMAA $/ \mathrm{kg}$ $\mathrm{bw} /$ day for another 5-7 weeks, were given a single oral infusion $(1 \mathrm{mg} / \mathrm{kg}$ bw) of L-DOPA with a peripheral decarboxylase inhibitor after 6-8 weeks of BMAA treatment. Within 30 minutes these two animals showed selective recovery of marked facial movement and spontaneous activity (Spencer et al., 1987a). This study shows that high doses of BMAA can give rise to neurological symptoms similar to those described in the Guam ALS-PDC complex. The study has been critizised for using unrealistic dosages, since it has been argued that it is not possible for humans to ingest the doses of BMAA given to the monkeys (lowest dose $100 \mathrm{mg}$ BMAA/kg bw/day) by consuming cycad flour.

Perry et al. (1989) performed a similar study to that of Spencer et al. (1987a), instead using female CD-1 mice as test animals. As it has been speculated that BMAA acts by interacting with the NMDA receptor, this study included treatments with MK-801 ((+)-5-methyl-10, 11-dihydro$5 \mathrm{H}$-dibenzo[a, d]cyclohepten-5, 10-imine maleate), a potent NMDA receptor antagonist. The study comprised four groups of mice, two of which were given DL-BMAA $\mathrm{HCl}$ in water solution by gavage, daily or every second day, and two of which were control groups given water by gavage in the same regime. The BMAA-treated mice received $500 \mathrm{mg} / \mathrm{kg}$ daily for 18 days, then $500 \mathrm{mg} / \mathrm{kg}$ every second day over 28 days and finally $1000 \mathrm{mg} / \mathrm{kg}$ every second day over a further 30 days. The study continued for 11 weeks, therefore resulting in a total dose of BMAA hydrochloride of around $31 \mathrm{~g} / \mathrm{kg}$, half of which was the L-isomer of BMAA (about $435 \mathrm{mg} / \mathrm{mouse}$ ). One of the groups of BMAA treated mice and one of the control groups were given daily subcutaneous injections with MK801 , in an effort to see if this NMDA-receptor antagonist could protect BMAA-treated mice against potential neurotoxic effects of BMAA. The dosage of MK- 801 was $2 \mathrm{mg} / \mathrm{kg}$ for the first 12 days but as this dose produced ataxia, weight reduction, excitement and falling in the dosed mice, 
and some mice died, the dosage was reduced to $1 \mathrm{mg} / \mathrm{kg}$ for the rest of the treatment period.

All mice given BMAA appeared healthy throughout the experiment, maintained weight and exhibited no behaviour abnormalities. At the end of the study, after 11 weeks, mice were sacrificed and brain and vertical column tissues isolated for biochemical and histological studies. BMAA had no influence of the mean contents of dopamine and its metabolites in striata, neither were the levels of noradrenaline, serotonin or 5-HIAA affected. Of the amino acids analysed for in cortices (taurine, asparagines, glutamine, glycine, and GABA), only the level of glycine was reduced in mice fed BMAA.

Histological examination revealed no pathological changes in the brain and the spinal cord in any of the mice. There was no evidence of neurolan loss, gliosis or neurofibrillary degeneration in cerebral cortex, hippocampus, striatum or substantia nigra. Anterior horn cells showed no evidence of chromatolysis, intracytoplasmic inclusions or neurofibrillary tangles. Axonal swellings were not seen. Based on these observations, the authors concluded that BMAA in cycad seeds is unlikely to be responsible for the ALS and PDC disease previously common on Guam. It was suggested to the authors that the use of a racemic mixture of BMAA could have prevented them from obtaining results similar to those of Spencer et al. (1987a), as the non-toxic D-isomer might have inhibited an interaction of the toxic L-isomer with the NMDA receptor. This possibility was not considered to be likely since renal tubular reabsorption of the D-isomer fails (Silbernagl, 1988) and there is no evidence that the Disomer binds more readily to the NMDA receptor than does L-BMAA.

Seawright et al. (1990) studied the effect of i.p. injections of a BMAA racemate and the L-BMAA enantiomer in young rats. BMAA, as the hydrochloride salt, was dissolved in $\mathrm{NaOH}$ to give a neutral solution. Doses of the L-form were 6-14 micromol/g body weight. The amounts of racemate given to the rats ranged from $0.35 \mathrm{mg} / \mathrm{g}$ to about $4 \mathrm{mg} / \mathrm{g}$ body weight. In the study, functional disturbances were observed in young rats intraperitoneally injected with 1-4 mg BMAA racemate per $g$ body weight, or 0.5-3 mg L-BMAA per g body weight. Both the recemate and the L-form of BMAA produced acute signs of cerebellar dysfunction and degeneration of cerebellar stellate, basket, Purkinje and Golgi cells, but did not influence granule cells. Ocasionally degenerative changes were seen in cerebellar roof nuclei. No other changes were found in the CNS. Thus, all cells showing degeneration were GABAergic (Seawright et al., 1990).

When administered subcutaneously to neonatal rat pups, BMAA resulted in changes in motor function and spinal cord chemistry, effects that were both dose and sex-dependent (Dawson et al, 1998). Twelve pups were injected with $500 \mathrm{mg} \mathrm{L}-\mathrm{BMAA} / \mathrm{kg}$ bw on postnatal day (PND) 5 (Group A), 24 pups were injected with $500 \mathrm{mg} / \mathrm{kg}$ bw on PND 2 and 5 
(group B), and 12 pups were injected with $100 \mathrm{mg} / \mathrm{kg}$ bw on PND 2 and 5 (group C). The control group consisted of 24 animals that were injected with phosphate buffered saline. The animals were weaned on day 21 , and sacrificed on day 101. Neurobehavioral testing was performed on postnatal days 13, 15, 22, 34, 43, 57 and 84 and included forepaw suspension, negative geotaxis, righting reflex, hindlimb splay, tail flick and open field behaviour. Hindlimb splay was significantly increased in females in group A. Males in groups A and B exhibited elevated quadrant crossings and rearing in the open field behaviour test.

Blood pressure was significantly elevated in female rats of group B. Males in groups A and B had elevated spinal cord 5-HIAA content. Glu and Asp content in the spinal cord was elevated in the female of the Cgroup. Monoamines were altered in the cerebellum, mediobasal hypothalamus and hippocampus. BMAA induced an increase in $\alpha$-adrenergic binding sites in the cerebellum. The results demonstrated that BMAA given neonatally can produce changes in motor function and spinal cord neurochemistry. Neonatal exposure to BMAA resulted in permanent changes in adult neurochemistry that may be related to reorganisational effects induced by toxin-mediated neuroplasticity in developing neurons. However, the authors concluded that neonatal BMAA did not result in a pattern of neurochemical changes consistent with the changes reported in postmortem tissue from ALS patients. The spinal cord atrophy in males and behavioural changes observed suggests some degree of motor system impairment. It was concluded that it is important to investigate the interaction of aging with these neurochemical changes (Dawson et al, 1998).

In order to test the hypothesised role of BMAA in neurodegeneration, adult male CD-1 mice were daily fed $28 \mathrm{mg}$ BMAA/kg body weight for 30 days, a dose chosen to reproduce environmental levels (Cruz-Aguado et al, 2006). The dose was chosen to be similar to the amount that a human who consumes two $500 \mathrm{~g}$ bats (flying foxes) would ingest (Cox et al., 2003). There were two experimental groups, one group fed BMAAcontaining pellets ( $1 \mathrm{mg}$ per pellet) and one control group, each group consisting of 7 animals. The pellets containing BMAA were given at 5 $\mathrm{pm}$ in the absence of standard food. In the same manner, pellets without BMAA were given to control animals. No BMAA-containing or control pellets were found uneaten, which indicated that all BMAA that was supplied was ingested. In the morning standard chow was supplied. The mice were tested with a battery of behavioural tests, including evaluation of motor coordination, reflexes, locomotion, muscular strength and memory. Neuronal numbers and glial response in the spinal cord and brains were examined to assess if BMAA triggered cell death or inflammatory glial responses in the CNS.

Dietary exposure to BMAA did not induce any observable change in the motor performance or anxiety levels of the treated mice, as measured by the rotarod test (motor coordination), leg extension (motor neuron- 
mediated reflexes), stride length (locomotion), open field behaviour (locomotion, anxiety level) and wire hang (muscular strength). In a memory task consisting of a radial arm water maze, there were no significant changes in BMAA treated mice as compared to control. Neuropathological examination of brains and spinal cords from BMAA-fed animals did not show any sign of neuronal damage or activation of apoptosis. Since lower motor neurons are neuronal populations targeted by ALS-PDC and cycad toxicity they assessed the ventral horn neurons by Nissl staining and immunoreactivity to ChAT (a marker of cholinergic alpha motor neurons), but none of the methods showed any changes in the number of lower motor neurons in BMAA-fed animals. The authors note that in previous experiments with the same battery of behavioural tests and histological protocols, mice fed cycads that contained no BMAA or MAM showed motor and cognitive deficits, as well as decreased neuronal numbers and signs of apoptosis in the ventral spinal cord and other CNS areas (Wilson et al, 2002). It was also noted that pharmacokinetic studies by Duncan et al (1991) had shown that BMAA reaches potentially toxic levels in the CNS when administered at dose levels that are orders of magnitude higher than environmentally available levels and that BMAA is a very weak glutamate agonist that gives rise to damage at levels in the millimolar range, whereas most excitotoxins are active in the micromolar range. Overall, the findings in this study did not support a causal role for BMAA in ALS-PDC or other neurodegenerative disease, according to the authors (Cruz-Aguado et al., 2006)

\subsection{Studies on infusion of BMAA directly into brain or brain ventricles}

Intracerebroventricular (i.c.v.) injection of 1-10 micromole DL-BMAA in mice induced a syndrome of ataxia, ptosis, scratching, jumping, myoclonic jerks, clonic muscle spasms and tonic seizure. The effects were neither influenced by an intraperitoneal injection of the competetive Nmethyl-D-aspartate (NMDA) receptor antagonist D(-)-4-(3phosphonoprop-2-enyl)-piperazine-2-carboxylate, nor by intracerebroventricular co-administration of the competetive NMDA/KA receptor antagonist gamma-D-glutamylamino-methylsulphonate at a dose which selectively blocks KA-induced excitotoxicity. However, pretreatment with the AMPA (quisqalate)/kainate antagonist 1-(aminophenyl)-4methyl-7, 8-methyendioxy-5H-2, 3-benzodiazepine decreased the incidence of clonic seizures. This observation could indicate an involvement of the AMPA/quisqualate subtype of excitatory amino acid receptors in acute BMAA toxicity (Smith and Meldrum, 1990).

Following intracisternal injections or local intracerebral administration of test substance into substantia nigra of rats, L-BOAA and L-BMAA 
were found to affect central monoamine neurons, although potency and specificity were low. In vitro studies on synaptosomal preparations indicated that though both BOAA and BMAA affect dopamine (DA) and noradrenaline (NA) neurons, the effects on DA neurons seem to be exerted through nonselective mechanisms whereas both BOAA and BMAA seem more selectively to NA neurons (Lindström et al., 1990).

Neurotoxic properties of L-BMAA after chronic intracerebroventricular (i.c.v.) administration of L-BMAA (500 microg/day) for up to 60 days were investigated in rats. According to the authors the results showed that intracerebroventricular administration of BMAA at high doses to adult rats influenced both cholinergic and glutamatergic systems and produced excitatory behavioural changes such as splay, jerking movements and rigidity. Symptoms remained for at least 6 days after the repeated administration, but diminished by day 10 after the end of treatment. Concentrations of L-BMAA less than $300 \mu \mathrm{M}$ produced little overall neuronal damage, while $3 \mathrm{mM}$ consistently destroyed virtually the entire neuronal cell population. Notably, L-BMAA was neurotoxic only in the presence of bicarbonate. The study supports the hypothesis that LBMAA neurotoxicity may be mediated through AMPA receptors, and that BMAA may be able to stimulate both NMDA and non-NMDA Glu (AMPA) receptors (Rakonzay et al, 1991).

In a similar study, rats were intracerebroventricularly administered LBMAA (10 nanomoles) or phosphate buffered saline (PBS). Some rats received the NMDA-glutamate-receptor antagonist 2-amino-5 phosphonovalerate (AP5) alone or in combination with BMAA. Rats were observed for 3 or 7 days, before being sacrificed, and brains dissected for analysis. Rats exposed to L-BMAA grew more slowly and contained less brain L- $\left({ }^{3} \mathrm{H}\right)$ glutamate binding sites than rats receving injection of phosphate buffered saline (PBS). Rats receving BMAA also developed a characteristic behavioural pattern, including loss of mobility and keeping their heads in a tilted position with occasional side to side movements. Rats receiving BMAA together with AP5 behaved similarly to rats receving PBS with respect to their L- $\left({ }^{3} \mathrm{H}\right)$ glutamate binding activity and behaviour. The study showed that the toxic effects of L-BMAA are mediated by NMDA-subtype L-glutamate receptors. Furthermore, the drug-induced decrease in number of glutamate receptors suggest an involvment of altered glutamate receptor status in the manifestation of L-BMAA neurotoxicity (Chang et al., 1993).

Behavioural changes have also been observed when $500 \mu \mathrm{g}$ BMAA was infused into the lateral ventricle of rats. The treatment induced splay, rigidity and clonic convulsions accompanied by epileptiform EEG. Concommitant exposure to NMDA receptor antagonists of different types indicated that L-BMAA may act upon both NMDA and non-NMDA receptors (Matsuoka et al., 1993). 
In a study on the influence of intracerebroventricularly injected BOAA and BMAA on different activity-dependent factors in the adult rat brain, Anderson et al. (1997) showed that both amino acids induced changed mRNA expression of activity-dependent factors and biomarkers for neuronal stress. One hour after BOAA and BMAA injections, a transient but pronounced increase in mRNAs for brain-derived neurotrophic factor, nerve growth factor, c-fos and $h s p 70$ (heat-shock protein 70) was observed. A rapid activation of both the c-fos gene and the $h s p 70$ gene is noted after seizures, brain trauma and ischaemia. Although the authors conclude that there is insufficient evidence to link exposure to the glutamate analogues BOAA and BMAA to neurological disorders, they may be used as experimental tools to investigate mechanisms that regulate gene expression.

\subsection{In vitro studies}

Several studies have shown that BMAA is toxic to neuronal cells in culture (Spencer et al., 1987a; Duncan, 1991). In 1987 Ross and co-workers reported that the acute neurotoxicity of the amino acids BOAA and BMAA might selectively be blocked by specific glutamate receptor antagonists. Administration of BOAA and BMAA to neonatal mouse cortex explants rapidly induced postsynaptic vacuolisation and neuronal degeneration characterised by dark and shrunken cells. BMAA-induced changes were antagonised selectively in a concentration-dependent manner by the NMDA glutamate receptor antagonist 2-amino-7-phosphonoheptanoic acid. This substance did not protect against damage induced by BOAA, whereas cis-2, 3-piperidine dicarboxylic acid, an antagonist of another glutamate receptor type, did. The glutamate receptor antagonists glutamic diethyl ester and streptomycin failed to protect the explants from destructive action of either amino acid. The authors conclude that the data indicates that BMAA is likely to act indirectly, possibly through a metabolite, and exerts its action predominantly via interaction with the NMDA glutamate receptor (Ross et al., 1987).

However, Copani et al. $(1990,1991)$ reported that the action of LBMAA is not restricted to NMDA receptors, as it also involve 'metabotropic' glutamate receptors coupled to inositol phospholipid hydrolysis. Thus, when the effect of L-BMAA on inositol phosphate formation was studied in primary cultured striatal neurons L-BMAA induced inositol phosphate formation via a direct action at the glutamate metabotropic receptors (Manzoni et al., 1991). Therefore the neurotoxic properties of L-BMAA might implicate the activation of the glutamate metabotropic receptor in association with the NMDA receptor.

The pharmacological specificity of the mGluR1alpha-subtype of the metabotropic glutamate receptor was examined in competition experi- 
ments in a cloned baby hamster kidney cell line by measuring displacement of $\left[{ }^{3} \mathrm{H}\right]$ glutamate binding and inositol phosphate (PI) hydrolysis. PI hydrolysis was maximally stimulated by L-BMAA; the relative order of potency of tested substances was quisqualate $>$ glutamate $>$ ibotenate $>t-$ ACPD > BMAA. In competition experiments $\left[{ }^{3} \mathrm{H}\right]$ glutamate was displaced by tested substances in a rank order of potency similar to that found for stimulation of PI hydrolysis (Thomsen et al., 1993).

A few years earlier Zeewalk and Nicklas (1989) noted that L-BOAA and L-BMAA cause exitotoxic damage in chick retina, BOAA through a non-NMDA receptor of the glutamate receptor type and BMAA through the NMDA receptor. BMAA had a potency similar to glutamate. In subsequent studies on embryonic chick retina it was investigated whether nitric oxide pathways exist in the retina and could be linked to excitatory amino acid-induced increases in cyclic guanosine monophosphate (cGMP). It was shown that BMAA, in contrast to BOAA and domoic acid, had no influence on cGMP. Glutamate and kainate increased the cGMP content 2-3-fold (Zeewalk and Nicklas, 1994).

Further demonstration of the difference in toxic potency between LBOAA and L-BMAA was obtained in an in vitro model consisting of sagittal slices of mouse brain (Pai et al., 1993a, 1993b). Incubation of such slices with BOAA $(1 \mathrm{pM})$ resulted in significant leakage of lactate dehydrogenase $(\mathrm{LDH})$ and potassium from the slices into the medium. The leakage was accompanied by inhibition of mitochondrial NADHdehydrogenase. Under similar conditions L-BMAA induced LDH leakage only at very high concentration of the amino acid $(1 \mathrm{mM})$. Although L-BMAA induced LDH leakage there was no inhibition of mitochondrial NADH-dehydrogenase activity.

Serum from patients with motor neurone disease induced a 2-3-fold increase in thyrotrophin-releasing hormone (TRH) in fetal rat brain stem cultures exposed to such serum, whereas sera from several other neurological disease states, including muscle wasting disorders, did not have this effect. L-BMAA produced similar dose-related and stereospecific effects on the cultures ability to synthesise TRH (Lewis et al., 1990).

Electrophysiological and receptor binding techniques were used to determine whether BMAA can modify the activity of NDMA receptors. In these studies neither L-BMAA nor DL-BMAA evoked membrane currents when applied in concentrations up to $3 \mathrm{mM}$ to hippocampal neurons. DL-BMAA, but not L-BMAA, reversibly potentiated the amplitude of NMDA-activated currents and DL-BMAA produced a concentrationdependent displacement of specific $\left[{ }^{3} \mathrm{H}\right]$ glycine binding in whole mouse brain synaptic membranes. The experiments implies that DL-BMAA is able to interact with the strychnine-insensitive glycine modulatory site (SIG) located on the NMDA receptor complex. However, BMAA appears to have lower affinity for the SIG than other active agents such as glycine. A determination of whether BMAA acting at the SIG plays a role in 
BMAA-mediated neurotoxicity requires further experimental work. Since BMAA is significantly less potent than glycine at the SIG, this mechanism would only be important under conditions in which glycine concentration would be very low. Data from other studies (McNamara and Dingledine, 1990) suggest that the interaction of BMAA with $\mathrm{NaHCO}_{3}$ is of primary importance for producing neurotoxicity with possible actions of BMAA at the SIG confined to conditions where the extracellular concentration of glycine is low (Allen et al, 1995).

This type of observation has led to the speculation that it is a modified BMAA molecule that is the bioactive compound. Myers and Nelson (1990) have shown that BMAA reacts with dissolved carbon dioxide to form two carbamate compounds at physiological $\mathrm{pH}$, temperature and bicarbonate concentrations (for details, see chapter 5.3). The reversible reactions of BMAA with dissolved carbon dioxide were monitored with ${ }^{13} \mathrm{C}$ NMR. At $37^{\circ} \mathrm{C}$ and $\mathrm{pH} 7.4$, the fraction of BMAA existing as the $\alpha-$ $\mathrm{N}$-carboxy adduct is 0.22 while the fraction of BMAA existing as the $\beta$ $\mathrm{N}$-carboxy adduct is 0.09 . Although both adducts could be implicated in bicarbonate-dependent neurotoxicity of BMAA, the $\beta$-N-carboxy adduct shares structural characteristics with the appropriate endogenous NDMA receptor ligand glutamic acid.

Weiss and Choi (1988) noted that BMAA produced neurotoxic and neuroexcitatory effects in murine cortical cell cultures only when physiological concentrations of bicarbonate were available in the extracellular medium. It was suggested that bicarbonate may interact noncovalently with BMAA to produce a molecular configuration that activates glutamate receptors.

BOAA is implicated in the pathogenesis of lathyrism. BOAA is a dicarboxylic amino acid, and can, like other such amino acids, produce neuroexcitatory and neurotoxic effects on central neurons and can induce seizures in rodents. Less potent than BOAA, single doses of BMAA can also induce seizures in rodents. In explants of the spinal cord or frontal cortex, BMAA can produce excitotoxic neuronal degeneration that can be attenuated by N-methyl-D-aspartate (NMDA) antagonists. Because BMAA lacks the omega-acidic or electronegative moiety characteristic of other excitatory amino acids (having instead a positively charged betaamino group) this excitotoxic action is curious.

In cell cultures of cortical neurons, Weiss and Choi (1988) found that the neurotoxicity of BMAA depended on the concentration of $\mathrm{HCO}_{3}{ }^{-}$with little toxicity in the presence of less than $6 \mathrm{mM} \mathrm{HCO}_{3}{ }^{-}$(Weiss and Choi, 1988). $\mathrm{As} \mathrm{HCO}_{3}{ }^{-}$interacted specifically with BMAA the cytotoxicity is unlikely to be due to a direct effect of $\mathrm{HCO}_{3}{ }^{-}$on postsynaptic glutamate receptors and on neuronal viability in general. None of the other excitatory amino acid compounds studied, including BOAA and NMDA, required $\mathrm{HCO}_{3}{ }^{-}$to give rise to neuronal injury. The interaction between BMAA and $\mathrm{HCO}_{3}{ }^{-}$cannot be explained by an ability of $\mathrm{HCO}_{3}{ }^{-}$to reduce 
the cellular uptake or metabolism of BMAA. BMAA incubated in cultures without $\mathrm{HCO}_{3}{ }^{-}$was not toxic, but was fully available for subsequent rapid activation by addition of $\mathrm{HCO}_{3}{ }^{-}$. This interaction was not restricted to the toxic effects of BMAA, but extended to its neuroexcitatory effects. The simplest explanation is that $\mathrm{HCO}_{3}{ }^{-}$interacts directly with BMAA to produce a threedimentional structure suitable for glutamate receptor activation. Structure activity analysis of NMDA agonists suggests a threepoint association between agonists and receptor, with the agonist usually presenting a positively charged amino group, an alpha-carboxylate group and an omega-acidic group. A noncovalent interaction between the positively charged beta-aminogroup of BMAA and $\mathrm{HCO}_{3}{ }^{-}$could act both to negate the positive charge of the amino group and to add a moiety that approximates the omega-acidic group of other glutamate agonists.

Weiss et al. (1989a) investigated the toxicity of L-BMAA and LBOAA on cultured mouse cortical neurons in the presence of physiological concentrations of bicarbonate (Fig. 3). Bicarbonate was considered a required cofactor for BMAA neurotoxicity. A $24 \mathrm{~h}$ exposure to $10 \mu \mathrm{M}-$ $3 \mathrm{mM}$ BMAA or to $300 \mathrm{nM}-100 \mu \mathrm{M}$ BOAA induced concentration dependent neuronal degeneration without glial damage. The neurotoxic $\mathrm{EC}_{50}$ for BMAA was about $1 \mathrm{mM}$ and for BOAA about $20 \mu \mathrm{M}$.

Glutamate<smiles>[NH3+]C(CCC(=O)[O-])C(=O)[O-]</smiles>

BMAA<smiles>C[C@H](CN[C@@H]([NH3+])C(=O)[O-])C(=O)[O-]</smiles>

BOAA<smiles>[NH3+]C(CNC(=O)C(=O)[O-])C(=O)[O-]</smiles>

Fig 3. A hypothetical non-covalent interaction of BMAA and bicarbonate could mimic glutamate and become an agonist of the NMDA receptor (adapted from Weiss and Choi, 1988). 
Continued research into the BMAA bicarbonate interaction revealed that bicarbonate potentiates the ability of BMAA to open NMDA (N-methylD-aspartate) receptor-acitvated channels in isolated membrane patches (Weiss et al., 1989b). Also, the neurotoxic and neuroexcitatory effects of two structural analogues of BMAA, DL-2, 4-diaminobutyrate and DL-2, 3 -diaminopropionate were potentiated by bicarbonate. The fact that the single-channel conductance activated by the BMAA bicarbonate complex is similar to that induced by NMDA or glutamate argues against bicarbonate activation of the channel itself. However, it cannot be excluded that bicarbonate converts the NMDA receptor into a form that recognizes BMAA and related compounds, but the ligand specificity of the bicarbonate effect is most consistent with the hypothesis that bicarbonate interacts at the level of certain ligands rather than at the level of the receptor-channel complex. (Fig.4)

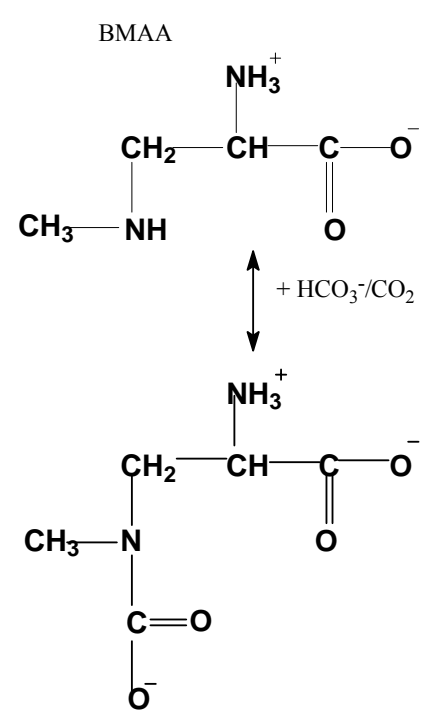

Fig. 4. A carbamate adduct of BMAA may resemble a glutamate agonist. (Adapted from Weiss et al, 1989b)

When $\left[{ }^{3} \mathrm{H}\right]$ glutamate binding to rat brain synaptic junctions were studied in vitro, L-BMAA was inactive in the absence of bicarbonate, but blocked $\left[{ }^{3} \mathrm{H}\right]$ glutamate binding with an $\mathrm{IC}_{50}$ of $1 \mathrm{mM}$ at $20 \mathrm{mM}$ ammonium bicarbonate. Identical inhibition was obtained in the presence of potassium bicarbonate (Richter and Mena, 1989).

As elevated $\mathrm{Ca}^{2+}$ concentrations in brain cells can lead to cell death and neurodisease, Brownson et al. (2002) evaluated the ability of BMAA to elevate the intracellular calcium concentration in dissociated newborn rat brain cells loaded with fura-2-dye. BMAA produced an increase in intracellular calcium levels in a concentration-dependent manner. Apart from being dependent on the extracellular calcium concentrations the increase was also dependent on presence of bicarbonate ions. The bicar- 
bonate dependence did not result from the increased sodium concentration or alkalinisation of the buffer. BMAA alone did not produce an increase in $\mathrm{Ca}^{2+}$ levels, suggesting the involvement of a product of BMAA and $\mathrm{CO}_{2}$, a beta-carbamate with a structure similar to other excitatory amino acids. The results support the hypothesis that neurotoxicity of BMAA is due to an excitotoxic mechanism involving elevated intracellular calcium levels and bicarbonate.

More recently Nedeljkov and co-workers (2005) investigated the effect of L-BMAA on input membrane resistance of leech Retzius nerve cells in the presence and absence of $20 \mathrm{mM}$ bicarbonate. The results showed that L-BMAA significantly reduced input membrane resistance in this model, leading to the conclusion that L-BMAA depolarises the cell by increasing membrane permeability and conductance. The effect was significantly higher in presence of $20 \mathrm{mM}$ bicarbonate than in standard Ringer solition. Also this result indicate that bicarbonate increases the excitatory effect of L-BMAA, presumably through formation of a compound that resembles the structure of major excitatory amino acids (Nedeljkov et al, 2005).

Transverse sections of mouse spinal cord cultures were used to determine whether the neurotoxic properties of BOAA and BMAA are optically specific. Cultures were initiated and the extent of pathological changes assessed. Cord explants were exposed for 10 minutes to 3 concentrations of L-BOAA $(0.056,0.28,0.56 \mathrm{mM})$, or for 60 minutes to 5 concentrations of L-BMAA $(0.19,0.38,0.81,1.62,3.2 \mathrm{mM})$. Additional experiments examined the response of cord explants treated for $3 \mathrm{~h}$ with each of the D-isomers (D-BMAA, $3.2 \mathrm{mM}$; D-BOAA, 0.28 and 0.056 $\mathrm{mM}$ ), with selected concentrations of L-isomers as controls. L-BOAA was a more potent acute neurotoxin than L-BMAA. At $0.81 \mathrm{mM}, \mathrm{L}-$ BMAA induced vacuolar changes within one minute. Similar changes developed within seconds following exposure to L-BOAA, even at the lowest concentration tested $(0.056 \mathrm{mM})$ (Nunn et al., 1987). The results showed that both L-BOAA and L-BMAA induce a characteristic pattern of postsynaptic neuronal edema and associated neuronal degeneration which is stereospecific for the L-isomers. It is likely that L-BMAA has an indirect excitotoxic action since a) L-BMAA lacks the dicarboxylic, diaminoacid structure of direct-acting glutamate-type excitotoxins, b) relative to L-BOAA larger doses and longer periods of exposure were needed for L-BMAA to induce comparable effects, and c) seizures induced by LBMAA are delayed in onset relative to L-BOAA, as supported by data of Polsky et al. (1972).

In other experiments BOAA, but not BMAA, increased basal cytosolic and $\mathrm{KCl}$-stimulated vesicular release of L-glutamate, but none of the tested compounds had any effect on dynorphin A(1-8)-like immunoreactivity release from these structures (Gannon and Terrian, 1989). 
In summary, it has been suggested that the neurotoxic effects of BMAA may be due to a metabolite since BMAA lacks the dicarboxylic acid structure characteristic of glutamate agonists, has less potency than BOAA and delayed time of action as compared to BOAA. Studies by Weiss and Choi (1988) showed that the cytotoxicity of BMAA is increased by bicarbonate and Nunn and O'Brien (1989) and Myers and Nelson (1990) indicated that nonenzymatic carbamate formation by reaction of BMAA with bicarbonate may explain the observation that BMAA and NMDA act at the same central nervous system receptors. To obtain information on enzymatic reactions of BMAA Hashmi and Anders (1990) conducted a study to investigate enzymatic reaction of BMAA with Lamino acid oxidase (L-AAO). L-AAO oxidises amino acids to the corresponding iminium ions. When BMAA was incubated with L-AAO in the presence of semicarbazide, BMAA was converted to iminium ion, which can be trapped as its semicarbazone or as its cyanide addition product. In the absence of semicarbazide, iminium ion undergoes hydrolysis to giv ammonia and the alpha-keto acid beta- $N$-methylaminopyruvate which can be trapped as its 2, 4-dinitrophenylhydrazone. In the absence of 2, 4dinitrophenylhydrazine, the alpha-keto acid undergoes oxidative decarboxylation to form $N$-methylglycine (Hashmi and Anders, 1991).

There are also data that seemingly contradict other observations. This section ends with a presentation of two further hypothesis introduced to explain the neurotoxic potency of cycad flour. The first of these two hypotheses emanates from observations reported by Duncan et al. (1992). Based on analytical data on the BMAA content in extracts from the female gametophyte tissue of $C$. circinalis, C. revoluae, and C. media; from common wheat flour and from 17 Guamanian cycad flour samples (in which there were much higher contents of BMAA in female gametophyte extracts than in processed cycad flour extracts), and further based on the toxicities of these extracts on cultured ventral mesencephalic and cerebellar granule cells, Duncan et al. (1992) came to the conclusion that BMAA is unlikely to be responsible for the toxicity. Three extracts from Guamanian cycad flour exerted a marked neurotoxic response, and a fourth extract was somewhat less toxic, whereas extracts prepared from the gametophyte tissue of the three seed types, household wheat flour, and extracts from 13 cycad flour samples elicited no neurotoxic response. There was no correlation between toxicity and BMAA content. The BMAA concentration in the extract was far below the level required to induce neurotoxicity. Studies, focusing on the toxic extracts, revealed high zinc concentrations in these extracts (Duncan et al., 1992). In parallel experiments, zinc was shown to be toxic to the studied cells and zinc toxicity mirrored the toxicity of the flour extracts. In contrast to high levels of BMAA, that have been shown to kill cortical neurons via NMDA and non-NMDA receptor activation, zinc was cytotoxic by other routes. Thus, whereas cytotoxicity of high concentrations of BMAA has been shown to 
be inhibited by NMDA and non-NMDA antagonists (MK-801 and 6cyano-7-nitroquinoxaline-2, 3-dione), zinc toxicity was not influenced by these receptor antagonists. Instead, EDTA protected the cultured cells to zinc toxicity. The zinc in the toxic extracts was assumed to have come from galvanized containers used for 'processing' of the cycad gamethophyte tissue. Duncan (1991) concludes his review on the role of cycad derived BMAA in the development of ALS-PSC in the Western Pacific in the following way:

\footnotetext{
"Although BMAA toxicity in humans is unknown, studies with nonhuman primates indicate that neither diet, topical application of a cycad poultice, or occasional medicinal use of the raw seeds is likely to deliver the doses of BMAA that appear necessary to elicit a neurotoxic response. It also appears highly unlikely that BMAA is the neurotoxic component responsible for the development of hindlimb paralysis in grazing animals, and the analogy seen in cattle and ALS-PDC in the western Pacific is poor".
}

The second hypothesis is originates in the observation that male CD-1 mice produced progressive motor and cognitive dysfunction after having been fed pellets made from washed cycad flour without appreciable amounts of cycad toxins (cycasin, methylazoxymethanol, BMAA and BOAA)(Wilson et al., 2002). Khabazian and co-workers (2002) tried to isolate and characterize the putative neurotoxins in cycad flour. The most toxic fractions of the washed cycad fluor to neural cells contained several sterol glucosides, of which beta-sitosterol beta-D-glucoside (BSSG) occurred in largest amounts, between 21-155 mg per $\mathrm{kg}$ washed cycad flour. BSSG was shown to be neurally active, evoking a rapid depolarising field potential and causing LDH release. BSSG also evoked a significant release of labelled glutamate, an effect that could be attenuated by AP5. As no or only low amounts of cycasin or MAM were present in the cycad flour, the authors believed that the molecule responsible for the cycad-induced neurodegeneration was BSSG which activates neural cells via glutamate release leading to NMDA receptor activation, culminating in cell death.

\subsection{Conclusions on BMAA as a natural toxicant in cycads and on studies of BMAA toxicity}

It has been established that the amount of BMAA in processed cycad flour is unlikely to be high enough to be the main cause of degenerative neurological disease observed in humans in Guam. Animal and in vitro studies indicate that diet is unlikely to deliver the doses of BMAA to humans that appear necessary to give rise to neurotoxic responses, unless 
humans are more susceptible to the compound than experimental animal, there are unknown depots of free or bound BMAA, or ingestion of low concentrations of BMAA during prolonged periods may be effective in inducing neurological damage by a mechanism that is as yet unknown.

Kinetic studies show that oral bioavailability of BMAA is good, and studies on tissue distribution show that the compound is distributed to the brain. It is unclear by what mechanism BMAA passes through the bloodbrain barrier. Some researchers suggest that influx to brain may be due to passive permeability, whereas others conclude that BMAA is transported by the large neutral amino acid carrier system. Pharmacokinetic studies indicate that levels of BMAA in the central nervous system that may be reached via environmental exposure are orders of magnitude lower than toxic levels.

In vitro studies indicate that BMAA is a weak glutamate agonist that may give damage at levels in the millimolar range, whereas most excitotoxins are active in the micromolar range. It has been suggested that bicarbonate/carbonate is required for BMAA to elicit interaction with neuronal receptors. This hypothesis is attractive but needs to be confirmed.

An attempt to isolate neurotoxins in cycad flour showed that the most toxic fraction contained several sterol glucosides. This led the authors to speculate that some of these compounds, which have the ability to activate neuronal cells resulting in cell death via glutamate release and receptor activation, may be responsible for cycad-induced neurodegeneration. The implication of this is that there may yet be undiscovered mechanisms and toxins to be recognised.

Present knowledge indicates that BMAA is unlikely to be the prime cause of degenerative neurological disease in man, unless unknown mechanisms are revealed that permit a higher degree of exposure to BMAA than is believed to occur today, or, alternatively, new mechanisms of action of BMAA are revealed. 


\section{Identity and physical and chemical properties of BMAA, and its chemical analysis}

\subsection{Identity}

BMAA, or $\beta-N$-methylamino-L-alanine (Figure 5), is an amino acid with a secondary amine in the side chain. The substance is also called $\alpha$ amino- $\beta$-methylamino propionic acid. Figure 5 , in addition to BMAA, presents some amino acids similar in structure to BMAA.

BMAA was discovered by Vega and Bell (1967) in extracts from Cycas circinalis. At that time it had been reported that cattle feeding on leaves of Cycadaceae species - palm-like plants found in the tropics and sub-tropics - could develop a neurological syndrome similar to that observed in humans consuming the legume Lathyrus sativus and referred to as "lathyrism".<smiles>[R]C(N)C(=O)O</smiles>

Structure of a general amino acid with the side chain $\mathbf{R}_{\mathbf{1}}$.

Side chains $\left(\mathbf{R}_{\mathbf{1}}\right)$ of different amino acids:

Lysine

Ornithine

$\alpha, \gamma$-diaminobutyric acid*

$\alpha, \beta$-diaminopropionic acid

Alanine

BMAA

$$
\begin{aligned}
& -\mathrm{C}_{\beta} \mathrm{H}_{2}-\mathrm{CH}_{2}-\mathrm{CH}_{2}-\mathrm{CH}_{2}-\mathbf{N H}_{2} \\
& -\mathrm{C}_{\beta} \mathrm{H}_{2}-\mathrm{CH}_{2}-\mathrm{CH}_{2}-\mathbf{N H}_{2} \\
& -\mathrm{C}_{\beta} \mathrm{H}_{2}-\mathrm{CH}_{2}-\mathbf{N H}_{2} \\
& -\mathrm{C}_{\beta} \mathrm{H}_{2}-\mathbf{N H}_{2} \\
& -\mathrm{C}_{\beta} \mathrm{H}_{3} \\
& -\mathrm{C}_{\beta} \mathrm{H}_{2}-\mathbf{N H}-\mathrm{CH}_{3}
\end{aligned}
$$

Figure 5. BMAA and some resembling amino acids. ( ${ }^{*} A$ well known neurotoxin occurring in some legumes.)

The toxic compound in L. sativus had been identified as $\beta$-oxalylaminoalanine (BOAA), also known as $\alpha$-amino- $\beta$-oxalylamino propionic acid. Researchers looking for the neurotoxin in cycads, therefore tried to identify BOAA in extracts from the cycad plant. They also looked for $\alpha, \gamma$ diaminobutyric acid, which was another known plant neurotoxin (Ressler et al. 1961). In extracts from Cycas circinalis seeds Vega and Bell found - by high voltage electrophoresis - a substance initially behaving simi- 
larly to $\alpha, \gamma$-diaminobutyric acid. However, further experiments revealed that the compound ha

$\mathrm{d}$ not earlier been described. After extensive chemical characterization, including studies based on paper chromatography, ninhydrin reactivity, and NMR analysis, the compound was identified as BMAA. The suggested structure was confirmed by chemical synthesis of the compound, and subsequent chemical confirmation that the synthesized BMAA showed all the characteristics of the extracted compound.

\subsection{Physical and chemical properties}

The BMAA isolated from Cycas circinalis seeds was identified as the L-isomer of the amino acid (Vega et al., 1968). Some physical properties and synonyms of L-BMAA are given in Table 1.

Table 1.Chemical and physical properties of BMAA (Vega et al., 1968; Duncan et al., 1989)

\begin{tabular}{ll}
\hline BMAA $=\beta$-N-methylamino-L-alanine & \\
\hline Synonyms: & L-BMAA \\
& $\beta$-methylamino-L-alanine \\
& $(+)$-L- $\beta$-N-Methyl- $\alpha, \beta$-diaminopropionic acid \\
& L- $\alpha$-amino- $\beta$ - methylamino propionic acid \\
& L-MeDAP (methylated $\alpha, \beta$-diaminopropionic acid) \\
& $15920-93-1$ \\
CAS Registry No: & $\mathrm{C}_{4} \mathrm{H}_{10} \mathrm{~N}_{2} \mathrm{O}_{2}$ \\
Molecular formula: & 118 \\
Molecular weight: & {$[\alpha]^{20}{ }_{\mathrm{D}}=+35^{\circ}(\mathrm{C}=1.15,5 \mathrm{~N} \mathrm{HCl)}$} \\
Specific rotation & $168^{\circ} \mathrm{C}$ for BMAA-HCl, $181-182^{\circ} \mathrm{C}$ \\
Melting point & $\mathrm{BMAA} \cdot \mathrm{HCl}$ is a white solid salt, soluble in watter. \\
Other properties &
\end{tabular}

To develop suitable methods of extraction, purification and identification of BMAA, a general understanding of how the $\mathrm{pH}$ influences the molecular charge state of amino acids is necessary. This knowledge might also be important for understanding the chemical reactions that might influence the biological activity of compounds. One example of such a chemical reaction is the potential for carbamate formation during specific conditions.

Neutral amino acids, such as alanine, are zwitter ions at neutral $\mathrm{pH}$. This means that the carboxyl group is deprotonated and the amino group protonated, resulting in the net charge being zero. This property is associated with the carboxy- and amino-group having different $\mathrm{pK}$ values, 2.3 and 9.9, respectively. Thus, the carboxy-group is protonated below $\mathrm{pH}$ 2.3 and the $\alpha$-amino group below $\mathrm{pH}$ 9.9. Lysine, an example of a basic amino acid, in addition contains a side chain with a primary amino group 
having a $\mathrm{pK}$ of 10.8. Such amino acids will have a protonated side chain at neutral $\mathrm{pH}$, and therefore a net positive charge.

BMAA contains a carboxyl group, a primary amine and a secondary amine. One might expect the molecule to behave similarly to the basic amino acids, for example lysine. However, early determinations of $\mathrm{pK}_{1}$, $\mathrm{pK}_{2}$ and $\mathrm{pK}_{3}$ for BMAA - where $\mathrm{pK}_{1}$ corresponds to the carboxyl group and $\mathrm{pK}_{2}$ and $\mathrm{pK}_{3}$ corresponds to the two amino groups - gave the values 2.1, 6.5 and 9.8 respectively (Vega et al., 1968). Latter investigations, using ionic strength control $\left(0.1 \mathrm{M}, 25^{\circ} \mathrm{C}\right)$, resulted in $\mathrm{pK}$ values of 6.63 and 9.76 for $\mathrm{pK}_{2}$ and $\mathrm{pK}_{3}$ respectively (Nunn et al., 1989; Nunn and O'Brian, 1989). The chemical laboratory at the National Food Administration in Sweden produced a titration curve of BMAA (Figure 6).

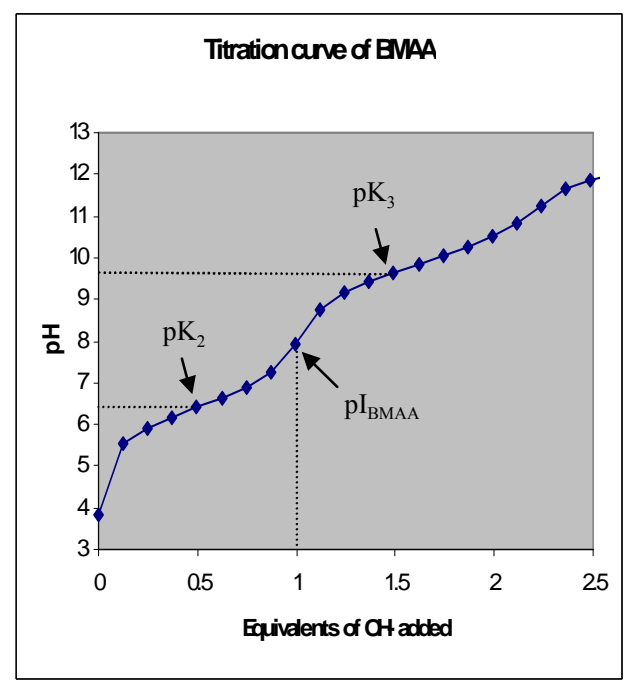

Figure 6. Titration curve of BMAA.

As can bee seen from the figure $\mathrm{pK}_{2}$ and $\mathrm{pK}_{3}$ are clearly separated, and evaluating the results graphically revealed $\mathrm{pK}$ values of 6.48 and 9.70 for $\mathrm{pK}_{2}$ and $\mathrm{pK}_{3}$ respectively (which is in accordance with the earlier findings) and a pI (isoelectric point) of 8.09. Evaluation of the data stoichiometrically resulted in similar values. Accordingly, the charge state of the main fraction of BMAA will be as follows:

\begin{tabular}{lll}
\hline pH & Charge state & Net charge \\
\hline$<2.1$ & fully protonated & $2+$ \\
$2.1-6.6$ & both aminogroups protonated & $1+$ \\
$6.6-9.8$ & one aminogroup protonated & 0 \\
$>9.8$ & fully deprotonated & $1-$ \\
\hline
\end{tabular}

Thus, at physiological $\mathrm{pH}(7.4)$ the main fraction of BMAA will have a neutral net charge. However, it should be noted that the compound is still a zwitter ion at this $\mathrm{pH}$, and "uncharged" BMAA will never be found in 
water solutions. However, other studies have suggested that a significant proportion of primary and secondary amines in biological samples can be present as carbamates (Schaefer, 2006). Moreover, these carbamates can be substrates for glucuronidation which may result in stable carbamate glucuronide metabolites. As pointed out by Schaefer (2006) glucuronidation is an important mechanism used by mammalian systems to clear and eliminate both endogenous and foreign compounds. However, glucuronidation is also a known phenomenon in plant systems (Klein et al, 2000). Glucuronic acid conjugation might play a role in the metabolism and the detoxification of BMAA, and may potentially influence the manifestations of toxicity of the compound. The formation of compounds such as these may also have an impact on analytical results. However, glucuronide conjugates of BMAA have as yet not been identified in biological material.

The $\mathrm{pK}$ values discussed above are referred to as "macroscopic" or "apparent" pK values. When coming to microscopic constants the detailed charge state of each group is considered. It has been suggested, based on calculations using "Martin's method" (Martin, 1979, Sayer and Rabenstein, 1976) that the doubly deprotonated form of BMAA is $86 \%$ $\alpha$-deprotonated and 14\% $\beta$-deprotonated (Figure 7) (Nunn and O'Brian 1989). Thus, the $\beta$-methylamino group would be the more basic of the two amino groups, but at neutral $\mathrm{pH}$ it is not only the $\alpha$-aminogroup that is deprotonated.

\begin{tabular}{cc}
\hline$\alpha$-deprotonated & $\beta$-deprotonated \\
\hline $\mathrm{CH}_{3}$ & $\mathrm{CH}_{3}$ \\
\hline & $\mathrm{NH}$ \\
$\mathrm{NH}_{2}^{+}$ & $\mathrm{N}$ \\
$\mathrm{I}$ & $\mathrm{CH}_{2}$ \\
$\mathrm{CH}_{2}$ & $\mid$ \\
$\mathrm{I}$ & ${ }^{+} \mathrm{H}_{3} \mathrm{~N}-\mathrm{CH}-\mathrm{COO}-$ \\
$\mathrm{H}_{2} \mathrm{~N}-\mathrm{CH}-\mathrm{COO}$ & $14 \%$ \\
$86 \%$ & \\
\hline
\end{tabular}

Figure 7. The two forms of doubly deprotonated BMAA, and their theoretically relative occurrence.

\subsection{Carbamates}

The earlier neurotoxicological studies indicated that BMAA may interact with NMDA-receptors (N-methyl-D-aspartate) (Allen et al., 1995, Ross et al., 1987, Spencer et al., 1987a). However, a direct binding of BMAA to the receptor was found unlikely. Instead it was suggested that a complex consisting of BMAA - having a bicarbonate/carbonate at the $\beta$ methylamino group - and the NMDA-receptor was formed. The formed complex would resemble the glutamate-NMDA-receptor complex (Weiss and Choi, 1988). However, available studies show that new compounds, 
rather than complexes, are formed when BMAA interacts with dissolved carbon dioxide. Firstly, it was shown in ${ }^{1} \mathrm{H}-\mathrm{NMR}$ studies that an $\alpha-N$ carboxy-adduct (I) was formed (Nunn and O'Brian, 1989) (Figure 8). Secondly, both (I) and a $\beta$ - $N$-carboxy-adduct (II) were studied by $13 \mathrm{C}$ NMR (Myers and Nelson, 1990) (Figure 8). In the latter paper LC-MS (using positive thermospray) was also used to detect a compound at $\mathrm{m} / \mathrm{z}$ 163 yielding a fragment at $\mathrm{m} / \mathrm{z} 119$. This would correspond to a carbamate of BMAA and loss of $\mathrm{CO}_{2}$ respectively. Quantitative studies revealed that - at physiological $\mathrm{pH}$, temperature and bicarbonate concentration - the fractions of BMAA existing as (I) and (II) were 0.22, and 0.09 respectively (Myers and Nelson, 1990). If these findings are relevant in biological samples $31 \%$ of the total BMAA would occur as carbamates and $69 \%$ as native BMAA.

\begin{tabular}{|c|c|}
\hline$\alpha$-N-carboxy-BMAA (I) & $\beta-N$-carboxy-BMAA (II) \\
\hline $\mathrm{CH}_{3}$ & $\mathrm{CH}_{3}$ \\
\hline 1 & | \\
\hline $\mathrm{NH}_{2}^{+}$ & $\mathrm{N}-\mathrm{COO}$ \\
\hline I & 1 \\
\hline $\mathrm{CH}_{2}$ & $\mathrm{CH}_{2}$ \\
\hline 1 & | \\
\hline $\mathrm{OOC}-\mathrm{NH}-\mathrm{CH}-\mathrm{COO}$ & ${ }^{+} \mathrm{H}_{3} \mathrm{~N}-\mathrm{CH}-\mathrm{COO}-$ \\
\hline $22 \%$ & $9 \%$ \\
\hline
\end{tabular}

Figure 8. The two suggested carbamate forms of BMAA, and the fractions of total BMAA they constituted experimentally at physiological $\mathrm{pH}$, temp. and bicarbonate concentration.

As only a deprotonated amino group can react with carbon dioxide to form a carbamate, the ratio of $\alpha-N$-carboxy-BMAA (I) to $\beta$ - $N$-carboxyBMAA (II) would mirror the prevalence of $\alpha$-deprotonated and $\beta$ deprotonated BMAA, respectively. Moreover, the formation of the $\alpha-N$ carboxy-BMAA (I) was found to be reversible, and on acidification the compound rapidly reverted to BMAA (Nunn and O'Brian, 1989). Most probably this would apply also for the $\beta-N$-carboxy-BMAA (II). It should be noted that the mentioned studies were performed only in water solutions of BMAA and bicarbonate. The structure of $\beta-N$-carboxy-BMAA is in Figure 9 compared to different amino acids. The relevance of the findings of carbamates are further discussed in the section on in vitro toxicity (section 4.6). 


\begin{tabular}{cc}
\hline Amino acid & Side chain \\
\hline BMAA & $-\mathrm{CH}_{2}-\mathrm{NH}_{2}^{+}$ \\
& $\mathrm{I}$ \\
$\beta$-N-carboxy-BMAA & $\mathrm{CH}_{3}$ \\
& $-\mathrm{CH}_{2}-\mathrm{N}-\mathrm{COO}$ \\
Glutamate \\
BOAA & I \\
$\mathrm{CH}_{3}$ \\
\hline
\end{tabular}

Figure 9. Side chains of $\beta$-N-carboxy-BMAA and some similar amino acids.

\subsection{Bound BMAA}

Since BMAA is not one of the 20 main amino acids ubiquitously occurring in proteins, it is often referred to as a "non-protein amino acid". However, it is still not clear whether BMAA sometimes may be incorporated into proteins, or may become associated to proteins. To deepen the discussion on this issue, it is needed to understand the analytical methods that have been used to obtain the data on BMAA content.

Although no t-RNA specific for BMAA has been identified, it cannot be excluded that BMAA may be incorporated into proteins via a t-RNA supposed to incorporate another amino acid. Another possibility for BMAA to become part of a peptide or a protein constituent would be through post-translational modification of protein amino acids. Theoretically, formation of BMAA could take this route, and free BMAA only be available after degradation of the modified protein or peptide.

The first evidence of BMAA becoming released from a bound state upon hydrolysis was published by Dossaji and Bell in 1973. Seeds and leaves from various cycad species were extracted with 50\% ethanol, and the extract analyzed quantitatively before and after hydrolysis $(6 \mathrm{M} \mathrm{HCl}$, $105^{\circ} \mathrm{C}, 17 \mathrm{~h}$ ) with an automatic amino acid analyzer (see section methods). In 6 out of the 9 species studied, hydrolysis increased the BMAA concentration by $0.5-43 \%$.

In a conference report from the same research group, $1 \%$ picric acid (trininitrophenol) was used for sample extraction, and extracts analyzed for BMAA with or without hydrolysis (Polsky et al., 1972). The authors give no information about the effect of the hydrolysis step, but in the discussion presented directly after the text of the publication some interesting information is given. In responding to questions from the audience, the authors state that the extra amount of BMAA obtained after hydrolysis was found in both the $1 \%$ picric acid and the $50 \%$ ethanol fractions, and conclude: "This bound form is therefore probably a low molecular weight compound" and "I would be surprised if it was found in protein". Scientists in the audience suggested that the increase was due to an artifact; e.g. methylation of $\alpha \beta$-diaminopropionic acid in the cycad extract 
during acid treatment. However, this suggestion is unlikely as no free $\alpha \beta-$ diaminopropionic acid was found in the nine investigated cycad species in the studies of Dossaji and Bell (1973). The possibility that BMAA might occur as a carbamate or even a carbamate glucuronide is further discussed in section 5.2 and 5.3.

Studies performed at the end of the 1980's used $0.1 \mathrm{M} \mathrm{HCl}$ for the extraction and claimed that "acid-labile conjugates" should have been detected (Duncan et al., 1989). However, in the analyzed cycad material they found similar levels of BMAA after this extraction as they found with $70 \%$ ethanol. Moreover, $0.1 \mathrm{M} \mathrm{HCl}$ during extraction is a significantly milder treatment than $6 \mathrm{M} \mathrm{HCl}, 105^{\circ} \mathrm{C}, 17 \mathrm{~h}$. Therefore, it could be questioned whether the acid-available fraction of BMAA described by Polsky et al. (1972) and Dossaji and Bell (1973) was detected in the work performed by Duncan et al. (1989).

Cox and collaborators have recently published a number of papers where acid hydrolysis has been included (Murch et al. 2004a, 2004b, Cox et al. 2005, Banack et al., 2006). In these papers 0.1M TCA was used for extraction of free BMAA and for precipitation of proteins. The precipitate was then subjected to hydrolysis in $6 \mathrm{M} \mathrm{HCl}$. Bound, as well as free BMAA, was found to be present in extracts from Cycads, brain tissues, cyanobacteria and flying foxes. The levels of bound BMAA were sometimes orders of magnitude higher compared to the levels of free BMAA. If BMAA occurs bound in such concentrations, the exposure to BMAA would be significantly higher than that indicated by the level of free BMAA. Furthermore, the presence of bound BMAA gave Cox and colleagues the opportunity to suggest mechanisms for biomagnification as well as for a slow release of BMAA, and a delayed toxic action. However, all determinations in these studies were performed with an HPLCfluorescence method using the derivatizing reagent AQC (see section 5.5.7), and other research groups or methods have yet not verified the findings.

\subsection{Methods of analysis}

\subsubsection{Qualitative methods used at the discovery of BMAA}

Several qualitative analytical methods were used in the paper describing the discovery and identification of BMAA (Vega and Bell, 1967). Fresh kernels from Cycas circinalis $(100 \mathrm{mg}$ ) were extracted with $50 \%$ ethanol. After centrifugation the supernatant was analyzed for its content of potential toxins:

High voltage ionophoresis was conducted on filter paper in buffer solutions of acidic or basic $\mathrm{pH}$ (all together 5 different values of $\mathrm{pH}$ ). Onedimensional paper chromatography was performed in 3 different systems 
using mixtures of organic solvents, water, acetic acid or in the presence of vapour of ammonia. For colour reactions in these methods ninhydrin and Erlich's reagent were used. $N M R$ in $\mathrm{D}_{2} \mathrm{O}$ showed three characteristic bands. On melting, the compound decomposed to give alanine and methylamine. Finally $I R$ spectrum showed characteristic absorption maxima. In all these methods synthesized BMAA gave identical results as the isolated unknown.

Similar methods as the ionophoresis and paper chromatography mentioned above have been used in later studies to confirm the identity of BMAA (e.g. Dossaji and Bell, 1973).

\subsubsection{HPLC and reaction with ninhydrin}

An "automatic Beckman amino acid analyzer" was used for the analysis of Cycad derived samples by Bell, Nunn and co-workers (Dossaji and Bell, 1973, Polsky et al., 1972). Samples were extracted with aquesous solutions of 1\% picric acid (Polsky et al., 1972) or 50\% ethanol (Dossaji and Bell, 1973). Quantitation was performed before and after hydrolysis using $6 \mathrm{M} \mathrm{HCl}, 105^{\circ} \mathrm{C}$ for $17 \mathrm{~h}$.

No details of the method were presented except for the chromatography (Dossaji and Bell, 1973). However, the principle was the same as on the classical system of Moore, Spackman and Stein (1958): Cationexchange chromatography on sulphonated polystyrene resins was used for the separation of amino acids at $\mathrm{pH} 4$ and $\mathrm{pH}$ 5.4. Detection was performed by post-column reaction with ninhydrin. In the presence of primary amines a blue color evolve from the ninhydrin reaction, and detection can be made with a photometer at $570 \mathrm{~nm}$. Since only primary amines are detected by the blue color, the method is relatively specific.

BMAA was found to elute immediately after lysine (Dossaji and Bell, 1973). Our laboratory found a similar, relatively high, retention of BMAA when standard solutions were analyzed in a corresponding system (at "Aminosyraanalyscentralen", Uppsala University); among the common amino acids only arginine eluted later.

No validation data at all were presented in the publications from the 1970's. The only hint on the variation for the method is the correlation between hydrolysed and non-hydrolysed samples. Especially when no extra BMAA was found in hydrolysates, this value was never below the non-hydrolysate. Instead it was equal to, or not more than $1 \%$ higher than the non-hydrolysate (in 7 out of 14 cases).

\subsubsection{HPLC using an FMOC derivative}

Kisby, Spencer and collaborators presented an HPLC method for BMAA using FMOC (9-fluorenylmethyl chloroformate) as derivatisation reagent and detection of the formed fluorescent adduct (Kisby et al., 1988, and 
Kisby et al., 1992). Samples analyzed were derived from cycads or were tissues from monkeys and rats supplied cycad material in the fed.

The analytical method was based on earlier work developed for general amino acid analysis (Einarsson et al., 1983). After aqueous extraction of the material, proteins were precipitated by $10 \%$ TCA before the derivatisation. Compounds were separated with reversed phase HPLC, and identified by excitation at $254 \mathrm{~nm}$ and emission at $315 \mathrm{~nm}$. The gradient program was empirically adjusted to resolve the FMOC derivative of BMAA from other amino acids.

In the paper from 1988 it is claimed that recovery of BMAA was "similar to $>98 \%$ ", although nothing was mentioned about the level of spiking. At reproducibility conditions CV\% was $2-7 \%$ for standards, nothing was mentioned about samples. In the paper from 1992 a sample spiked at $5200 \mu \mathrm{g} / \mathrm{g}$ flour gave a recovery of $54 \%$. The BMAA levels in cycad flour were approximately 7 times lower (average $5 \mu \mathrm{g} / \mathrm{g}$ ) than what was reported by Duncan and co-workers using PFPA derivatization and GCMS (average $35 \mu \mathrm{g} / \mathrm{g}$ ) (Duncan et. al., 1990). This fact was commented upon by the authors, who stated that the difference in result could be due to a shorter (i.e. not complete) extraction time used, or different detection techniques being employed.

Another study using derivatisation with FMOC was recently published by Montine et al. (2005). In this study brain samples were analyzed by the same protocol as described above having changed only the gradient program. Linearity was claimed from what would correspond to between $0.08 \mu \mathrm{g} / \mathrm{g}$ and $12 \mu \mathrm{g} / \mathrm{g}$, and at repeatability conditions CV\% was $<5 \%$. No more detailed data were presented regarding the validation, and nothing was mentioned about recovery or trueness. However, recovery or trueness might not be necessary for drawing conclusions from the work described by Montine since all samples from patients with Alzheimer disease or control subjects were negative. All samples were also spiked at $0.5 \mu \mathrm{g} / \mathrm{g}$ and were then determined positive. It should be noted, though, that the spiking was performed immediately before derivatisation, while normally it is preferred to spike the samples before the extraction. Another interesting fact is that in the published FMOC-papers no hydrolysis has been performed to release any bound BMAA.

\subsubsection{GCMS using a PFPA derivative}

Duncan and collaborators developed a GCMS-method using a three-step derivatisation procedure. They analyzed mainly materials obtained from cycad seeds according to the following approach (Duncan 1991, Duncan et al. 1989, 1990, 1991, 1992). For GC the polar functional groups of BMAA require derivatisation. If firstly an ester of the carboxylic acid group of BMAA is prepared, an extract can be cleaned up by extracting matrix components with dichloromethane (DCM) at neutral/acidic $\mathrm{pH}$, 
and then at a higher $\mathrm{pH}$, when the BMAA ester is deprotonated, the analyte can be extracted with DCM. The methyl ester was though found to be partly hydrolysed at the higher $\mathrm{pH}$. A sterically hindered 2-methyl-1propanol ester was found to be more stable, but also to be difficult to prepare directly from BMAA. Therefore a strategy of first forming the methyl ester and then the 2-methyl-1-propanol ester by transesterification was chosen. Finally the amino groups were acylated by PFPA (pentafluoropropionic anhydride) for GC compatibility, leading to the formation of an N, N-dipentafluoropropionyl, 2-methyl-1-propanol derivative of BMAA. GCMS quantitation was aided by a deuterium labeled internal standard synthesized at the laboratory.

From sensitivity data given in the publication describing the method, an LOQ of approximately $24 \mu \mathrm{g} / \mathrm{g}$ was estimated (corresponding to a signal to noise ratio of 10:1) (Duncan et al., 1989). The coefficient of variation was $3 \%$ under repeatability conditions at $146 \mu \mathrm{g} / \mathrm{g}$. Between assays the $\mathrm{CV} \%$ was found to be $5 \%$ at $467 \mu \mathrm{g} / \mathrm{g}$. In a later paper the LOD was estimated to $3 \mu \mathrm{g} / \mathrm{g}$ and the $\mathrm{CV} \%$ for duplicates to be $2-10 \%$ (Duncan et al., 1990).

Although an invaluable tool for achieving a correct result was used (the isotopically labeled BMAA), nothing was mentioned about trueness or recovery in the papers.

\subsubsection{CF-LIMS}

A method based on continuous flow liquid secondary ion tandem mass spectrometry (CF-LIMS) was developed by the same group that developed the GCMS/PFPA method described above (Wang et al., 1989). The method requires less derivatisation: only an ethyl ester of BMAA needs to be formed for the purpose of clean-up of extracts. This speeds up the method. However, the authors point out that the sensitivity is not even close to what was achieved by the GCMS-method. Moreover, the instrumentation is not commonly used today, and neither validation data nor data of occurrence of BMAA using the method was delivered.

\subsubsection{GCMS using an ECEE derivative}

Another derivative used for GCMS is the $N$-ethoxycarbonyl ethyl ester of BMAA (ECEE-BMAA) (Pan et al., 1997). This method was developed with the purpose to identify non-protein amino acids in cycad materials. Indeed 12 such compounds were identified, among them BOAA (Figure $9), \delta$ - $N$-oxalyl-ornitin (a BOAA analogue) and "cycasindene", a compound discovered by the group in cycad material. Since the results presented were only qualitative they do not add to the knowledge of occurrence of BMAA. 


\subsubsection{HPLC using an AQC derivative}

Cox and collaborators modified an HPLC method developed for general amino acid analysis by Cohen and Michaud (1993) to suit the analysis of BMAA. The method comprises derivatisation with 6-aminoquinolyl-Nhydroxysuccinimidyl carbamate (AQC) and detection by fluorescence (Banack and Cox, 2003a, 2003b, Cox et al., 2003; Murch et al. 2004a, 2004b; Cox et al. 2005, Banack et al., 2006).

Samples were extracted with $0.1 \mathrm{M}$ TCA in water in order to liberate free BMAA and to precipitate proteins. The free fraction was then subjected to the derivatisation procedure, in which primary and secondary amines or amino acids were reacted with $\mathrm{AQC}$ to form stable fluoroscent quinoline-labeled compounds upon release of N-hydroxysuccinimide.

Bound or "protein associated" BMAA was released from the precipitated protein fraction by acid hydrolysis using $6 \mathrm{M} \mathrm{HCl}$ at $110^{\circ} \mathrm{C}$ for $24 \mathrm{~h}$ (Murch et al. 2004a, 2004b; Cox et al. 2005; Banack et al., 2006). In some cases the hydrolysed extract was cleaned-up by solid phase extraction (SPE) using C18-cartridges (Murch et al., 2004a, 2004). However, how these SPE columns were used was not described. The hydrolysate, cleaned-up or not, was then subjected to derivatisation in a similar way to the free BMAA fraction.

An HPLC gradient using acetonitrile for a C18 HPLC column was then developed to resolve BMAA from other amino acids. Detection was performed by fluorescence with excitation at $250 \mathrm{~nm}$ and emission at 395 $\mathrm{nm}$.

In most of the publications of Cox and co-workers, LOD and LOQ were presented based on the response from standard solutions. Almost nothing was presented regarding accuracy and precision. Only in one paper a hint to the performance of the method was given: "BMAA recovery was $>96 \%$ for all tissue extractions", and "the coefficient of variation was $<5 \%$ for repeated analysis." (Murch et al., 2004b)

Until today all reported positive samples of the occurrence of BMAA in flying foxes, human brains and cyanobacteria have been generated by this single HPLC method only. Cox and coworkers claim they have used LCMS for the mentioned matrices to verify their findings. However, since hardly any results are shown from these LCMS verifications, all theories of biomagnification of BMAA, and having found a link to neurological diseases worldwide, up to now rely on the data produced by the single HPLC-method. 


\subsection{Development of an LC-MS/MS method and analysis of BMAA in cycads and cyanobacteria}

\subsubsection{Background}

An LC-MS/MS (Liquid Chromatography Tandem Mass Spectrometry) method for verification of the presence of BMAA in cyanobacteria and other matrices has been developed and used at the Swedish National Food Administration during 2005-2007. The reason was that all published results of positive findings of BMAA in cyanobacteria, flying foxes and human brain tissues were obtained by one analytical method only, the HPLC AQC method described in section 5.5.7, and presented in Table 2. The HPLC AQC method, along with most other methods developed for the analysis of BMAA, detects a chemical derivative of BMAA, and it is believed crucial to have access to a method detecting the native compound for confirmation of new findings. The HPLC AQC method may be regarded as relatively unspecific, since it detects not only all amino acids, but also any compound that reacts with the fluorescent derivatization reagent or exhibit fluorescence at the given settings. Retention time is thus the only criteria for identification, and at least 50 compounds in cyanobacteria can be detected with the method, many of them not resolved by base line separation. By contrast, tandem mass spectrometry (MS/MS) is generally regarded to be highly specific. For example in EU regulations for the analysis of illegal veterinary drugs, mass spectrometry is the only accepted method for confirmation. In MS/MS two daughter ions with a specific relative ratio are enough to verify the identity. For BMAA at least five specific daughter ions could be detected (se below).

\subsection{2. $\quad$ LC-MS/MS method}

When continuously infusing an aqueous solution of synthetic L-BMAAhydrochloride (from RBI, Sigma) into a triple stage quadrupole (Quattro Ultima, Micromass, Waters), using positive electrospray mode, the main mother ion was found to be $\mathrm{m} / \mathrm{z} 119$, corresponding to $[\mathrm{M}+\mathrm{H}]^{+}$. The main

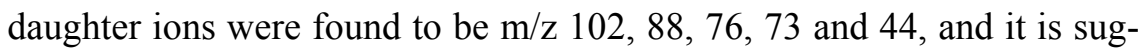
gested that the first four of these correspond to the loss of ammonia, methylamine, $\mathrm{CH} 2=\mathrm{N}-\mathrm{CH} 3$ and formic acid respectively. The transition $\mathrm{m} / \mathrm{z}$ $119>44$ is suggested to correspond to $[\mathrm{CH} 2=\mathrm{NH}-\mathrm{CH} 3]^{+}$. For the analysis of samples, all 5 daughters were recorded in MRM mode (multiple reaction monitoring). Several HPLC columns and mobile phases were tested, including e.g. a graphitized carbon column. The highest retention was found when hydrophilic interaction chromatography was employed, using a column with zwitter ions as functional groups (ZIC-HILIC, including a quaternary amine and sulphonic acid, SeQuant AB, Sweden). A mobile phase consisting of $65 \%$ acetonitrile and $35 \% 60 \mathrm{mM}$ formic acid re- 
sulted in sharp peaks. Sample extraction was performed essentially according to Cox et al. (2005). Cyanobacteria or cycad seeds were extracted with $0.1 \mathrm{M}$ TCA or $70 \%$ acetonitrile in water. For 'bound BMAA', acid hydrolysis was accomplished using $6 \mathrm{M} \mathrm{HCl}$ over night at $110^{\circ} \mathrm{C}$. Excess $\mathrm{HCl}$ was evaporated and extracts were passed through syringe filters. Ultrafiltration was used when necessary, before aliquots were injected into the LC-MS/MS system.

\subsubsection{Analysis of cycad seeds}

A sample of cycad seeds (homogenate of gametophyte tissue from seeds of Cycas micronesica, kindly provided by Dr. Bannack) was analyzed with the developed LC-MS/MS method. All five characteristic daughter ions were detected at the same retention time, and with the same relative ion ratios, as for a standard solution of BMAA. Moreover, for four of the daughter ions no other peak could be detected in the entire chromatogram. Only for the transition 119>73 one matrix peak could be detected. However, this peak was well separated from the BMAA peak. These results not only confirm the presence of BMAA in seeds of Cycas micronesica, but also demonstrate the extremely high specificity for BMAA of the developed method. In preliminary experiments, blank samples (pine kernels) were spiked with BMAA and analyzed. From these results it was concluded that the method would not be directly applicable to quantitative BMAA determination. This is most probably due to ion suppression, a common phenomenon in LC-MS/MS. To solve this problem either the crude extract has to be submitted to clean-up, or a suitable internal standard would be needed. The concentration of BMAA in the cycad seed was therefore not estimated. However, the signal from all detected peaks was at least 10-100 times higher than the noise level $(\mathrm{s} / \mathrm{n}>$ 10-100).

\subsubsection{Analysis of cyanobacteria}

To verify the occurrence of BMAA in cyanobacteria, we tried to obtain cyanobacteria samples as similar as possible to those used in the publication of Cox et al. (2005). Several of these samples were originally from Dr. Bergman at Stockholm University. She kindly provided us with similar samples. These samples were analyzed for free BMAA and in most cases, when the sample material was enough, also for bound BMAA after hydrolysis. In total 21 analyses were performed. BMAA could not be verified in any of these samples. Not even traces $(\mathrm{s} / \mathrm{n}>3)$ were detected. In order to interpret the negative findings, the detection capability of the method for these samples, i.e. the lowest concentration of BMAA that could be detected, needed to be determined. In the absence of an internal standard (se above), the approach chosen was to analyze each sample 
twice, one of them spiked at a low concentration of BMAA before extraction and/or the acid hydrolysis. From the results the detection capability was estimated for each sample, and ranged from 0.2 to $9 \mu \mathrm{g} / \mathrm{g}$ for free BMAA, and from 0.5 to $16 \mu \mathrm{g} / \mathrm{g}$ for bound or total BMAA.

It should be pointed out that fluorescence detection, as used in the previous studies, is generally a highly sensitive detection method. And, although no BMAA was detected in any of the cyanobacteria samples by LC-MS/MS, BMAA could still be present at lower concentrations than the estimated detection capability. Another possible explanation to the diverging results could be that although similar cyanobacterial samples to those of Cox et al. (2005) were analysed, they were not identical.

However, the reported wide distribution of BMAA in cyanobacteria reaching relatively high concentrations, $3-6000 \mu \mathrm{g} / \mathrm{g}$ in $95 \%$ of the samples analyzed by Cox et al. (2005), could not be confirmed by the developed LC-MS/MS method. Since there are uncertainties regarding the identity of the compound identified in earlier studies, and at least a potential risk that reported concentrations have been overestimated, any report that can confirm or refute the findings would be invaluable. This would also apply to the findings of BMAA in flying foxes and human brain tissues.

\subsection{Analysis of cyanobacteria with HPLC and the ninhydrin reaction}

An HPLC method based on the ninhydrin reaction for the analysis of amino acids is currently in use at "Aminosyraanalyscentralen", Uppsala University. The principle is similar to what is described in section 5.5.2. Standard solutions of BMAA and samples to be analysed for BMAA were sent from The Swedish National Food Administration to "Aminosyraanalyscentralen" in 2006. The samples were cycad seeds as described in section 5.6.3., and 8 of the cyanobacteria samples described in section 5.6.4. The presence of BMAA in the sample of cycad seeds was successfully confirmed. No BMAA could be detected in the cyanobacteria samples. However, no full validation was performed at "Aminosyraanalyscentralen", and the detection capability was estimated to approximately $100 \mu \mathrm{g} / \mathrm{g}$, i.e the method is significantly less sensitive than the LCMS/MS method described in section 5.6. 


\subsection{Conclusion on physical and chemical properties of BMAA and methods of analysis}

Most studies on analysis of BMAA have focused on the free form of the amino acid. Studies of $\mathrm{pK}$ values have revealed that at physiological $\mathrm{pH}$ the compound is a zwitter ion with a neutral net charge. However, it has been suggested that other forms than the native form may exist in biological samples. Dossaij and Bell discovered in 1973 that hydrolysis increased the BMAA content found by quantitative analysis between 0.5 up to $43 \%$ in seeds and leaves from cycad species. They concluded that this bound form probably was a low molecular weight compound. More recently Cox and coworkers have instead detected a protein bound portion of BMAA in several different matrices. The levels of this protein bound BMAA were sometimes reported to be orders of magnitude higher compared to the levels of free BMAA.

Another possible form of BMAA would be as a carbamate after reaction with dissolved carbon dioxide. Both $\alpha$-, and $\beta-N$-carboxy-BMAA have been detected in water solutions and have also been implicated to alter the physiological effects of BMAA, see section 4.6. The formation of carbamates was found to be reversible. However, carbamates of other amino compounds have been found to be substrates for glucuronidation, thereby forming stable metabolites. If BMAA may occur as carbamate or as a carbamate glucuronide in biological material remains to be proven.

Table 2 summarizes all published significant analytical methods for BMAA (see also section 5.5.1 to 5.5.7), and what matrices they have been used for. For cycad derived materials it is obvious that several methods based on various techniques have been developed and used. Generally, methods derived from many of these techniques would typically serve well for quantitative purposes. However, quantitative validation data were not satisfactorily reported for any method mentioned in table 2 or section 5.5.1 to 5.5.7. Therefore, all available quantitative data on BMAA in cycad derived materials should be used with care.

For all the other matrices, as well as for protein bound BMAA, all reported positive findings were based on one analytical method only. Confirmation or refution of the presence of BMAA in any of these matrices using another method - would be highly appreciated. Especially if a method with high specificity is used. Hitherto unpublished results from the Swedish National Food Administration have been inserted in table 2, see section 5.6 and 5.7. 
Table 2. Matrices analyzed for BMAA and analytical methods used (+ = positive findings; nd = not detected, i.e. negative findings)

\begin{tabular}{|c|c|c|c|c|c|c|c|}
\hline & $\begin{array}{l}\text { Qualitative } \\
\text { methods* }\end{array}$ & $\begin{array}{l}\text { HPLC } \\
\text { ninhydr. }\end{array}$ & $\begin{array}{l}\text { HPLC } \\
\text { FMOC }\end{array}$ & $\begin{array}{l}\text { GCMS } \\
\text { PFPA }\end{array}$ & $\begin{array}{l}\text { GCMS } \\
\text { ECEE }\end{array}$ & $\begin{array}{l}\text { HPLC } \\
\text { AQC }\end{array}$ & LC-MSMS \\
\hline $\begin{array}{l}\text { Cycad material: } \\
\text { seeds, leaves, flour }\end{array}$ & $+{ }^{1}$ & $+2,9$ & $+{ }^{3}$ & $+{ }^{4}$ & $+{ }^{5}$ & $+{ }^{6}$ & $+{ }^{8}$ \\
\hline Flying foxes & & & & & & $+{ }^{6}$ & \\
\hline Human brains & & & $\mathrm{nd}^{7}$ & & & $+{ }^{6}$ & \\
\hline Cyanobacteria & & $\mathrm{nd}^{9}$ & & & & $+{ }^{6}$ & $\mathrm{nd}^{8}$ \\
\hline Protein bound ${ }^{* *}$ & & & & & & $+{ }^{6}$ & \\
\hline
\end{tabular}

*Ionophoresis/electrophoresis, paper chromatography, NMR and IR

${ }^{\star *}$ In cycad material, flying foxes, human brains and cyanobacteria

${ }^{1}$ Vega and Bell, 1967, Dossaji and Bell 1973;

2 Dossaji and Bell, 1973, Polsky et al., 1972;

${ }^{3}$ Kisby et al., 1988, and Kisby et al., 1992;

${ }^{4}$ (Duncan et al., 1989, Duncan et al., 1990, Duncan 1991, Duncan et al. 1991, Duncan et al., 1992;

${ }^{5}$ Pan et al 1997:

${ }^{6}$ One or several of the following papers: Banack and Cox, 2003a, Banack and Cox, 2003b, Cox et al., 2003, Murch et al.

2004a, Murch et al., 2004b, Cox et al. 2005, Banack et al., 2006;

${ }^{7}$ Montine et al., 2005;

${ }^{8}$ Swedish National Food Administration, unpublished data, see section 5.6;

${ }^{9}$ Swedish National Food Administration, unpublished data, see section 5.7 


\section{Occurrence of BMAA i cycads}

As pointed out earlier in this report, BMAA was originally detected in cycads when the investigators searched for other toxic non-protein amino acids. In subsequent studies, Dossaji and Bell (1973) noted that slightly higher amounts of BMAA could be found in cycad seeds and leaves when the materials were hydrolysed before extraction and analysis, possibly indicating that the compound might occur both in free and bound form. However, these possibilities were not seriously explored until thirty years later when Murch et al. (2004a) actually claimed that the compound occur also in bound form. As pointed out in Chapter 5, the analytical techniques used in these studies may be questioned. If BMAA is confirmed to occur bound to proteins, there is no proof that it is incorporated into proteins. Its appearance in proteins may also be the result of postsynthetic modification of protein amino acids already built into the polypeptides. However, no alanine-methylating activity has been identified in cycads.

The preponderance of data on free BMAA, therefore, reflects that BMAA have been known to occur in free form in cycads, and investigators have looked for a non-protein amino acid. As a few studies performed in latter years reports the occurrence of both free and bound BMAA in such diverse organisms as cyanobacteria, plants, animals, and humans, it might be prudent to consider whether these are accurate observations or some type of artefact. As shown in Table 2 (chapter 5) all later studies that have reported bound BMAA in these organisms have used the same analytical techniques. The widespread occurrence of BMAA is not easy to understand in absence of data on cellular activities leading to incorporation of BMAA into proteins.

It is worth pointing out that, with exception of the early studies establishing the presence of BMAA in Cycas circinalis using direct analytical methods (Vega and Bell, 1967; Vega et al, 1968), subsequently published data have been obtained with methods determining the fluorescence of derivatized BMAA separated by GC or HPLC. Although the early studies of Bell and co-workers provided valuable data on BMAA levels in cycads, it has to be recognised that the sensitivity, specificity, and precision of the early analytical techniques were low in comparison with analytical techniques available today. In conclusion, it is only Vega and Bell (1967), Vega et al. (1968), Dossaji and Bell (1973), and Rosén at the National Food Administration in Sweden (this report) that actually have determined BMAA as such in the samples. 


\subsection{Cycad plants from the genus Cycas}

Seeds of $C$. circinalis contain significant amounts of BMAA at all stages of maturity (Duncan et al., 1988, 1989). As illustrated by Table 3, the compound is claimed to occur in both free and bound form (Banack and Cox, 2003a; Murch et al, 2004a). It should be noted, however, that the data available on free and protein-bound BMAA frequently were not obtained on the same material. Whereas the amount of protein-bound BMAA is fairly similar in various parts of the seed, the amount of free BMAA differs significantly between seed parts. The highest concentration is found in the developing reproductive organs (Banack and Cox, 2003a). The fleshy seed sarcotesta, which is the seed part consumed by animals such as flying foxes, has the lowest BMAA content, the gametophytes, or seed kernels, which are processed into flour by the Chamorro people, an intermediate level, and the outermost layer of the seed the highest level (also this consumed by flying foxes). In comparison to seeds, leaf tissue contains nearly ten times higher levels of protein-bound BMAA. The occurrence of free BMAA in leaves of other cycad species than those growing on Guam and being used for flour production is summarized in Table 4.

Although the amount of BMAA in cycad seeds varies between species, data on seeds of Cycas circinalis and Cycas rumphii collected from various areas of Guam, or from a botanical garden in Florida, United States, indicate that the variation in free BMAA content within a species is rather limited (Table 3). Also C. rumphii plants grown in other regions of the world contain similar levels of free BMAA.

BMAA has also been found in seeds of the two Cycas species used as medical preparations and linked to neurogenerative disease in other regions of the Pacific (C. revoluta and C. media ${ }^{3}$ ). Interestingly, the free BMAA content in seed samples of these two species was only one fourth of that in C. circinalis, independent of whether the seeds were collected from native cycad trees, or from botanical collections in the United States (Duncan et al., 1989). Although the BMAA levels reported by Duncan et al. (1989) were higher than those previously reported in C. revoluta and C. media by Polsky et al. (1972), and Dossaji and Bell (1973), possibly due to improved chemical methodology, they were still only one fourth of that in C. circinalis.

Comparing the levels of free BMAA in leaf tissue for estimation of dietary exposure with reported cases of cycad poisoning in animals where feed intake was known, indicates that cycad poisoning may occur at very low levels of free BMAA (corresponding to $0.56 \mathrm{mg} / \mathrm{kg}$ body weight).

\footnotetext{
${ }^{3}$ In a note added in proof, Duncan (1991) states that the Cycad species used by the native population of west New Guinea most likely is C. armstrongii and not C. media.
} 
Table 3. Amount of free, protein-bound and total BMAA in cycad plants of the genus Cycas used for flour production by the Chamorro people. For species identification, see footnote in Chapter 2.

\begin{tabular}{|c|c|c|}
\hline Product (number of samples) & BMAA content (mg/kg dry weight) & Reference \\
\hline $\begin{array}{l}\text { Female gametophyte of Cycas micronesica from Guam } \\
\text { and Hawaii }\end{array}$ & $\begin{array}{l}\text { Free: } 240^{*} \\
\text { Bound: } 81^{* *}\end{array}$ & $\begin{array}{l}\text { Banack and Cox, 2003a } \\
\text { Murch et al., 2004a }\end{array}$ \\
\hline \multirow{2}{*}{$\begin{array}{l}\text { Seed sarcotesta of Cycas micronesica from Guam and } \\
\text { Hawaii }\end{array}$} & Free: $9^{\star}$ & \multirow{2}{*}{$\begin{array}{l}\text { Banack and Cox, 2003a } \\
\text { Murch et al., 2004a }\end{array}$} \\
\hline & Bound: $89^{\star *}$ & \\
\hline \multirow{2}{*}{$\begin{array}{l}\text { Outer integument of seed sarcotesta of Cycas microne- } \\
\text { sica from Guam and Hawaii }\end{array}$} & Free: $1161^{*}$ & \multirow{2}{*}{$\begin{array}{l}\text { Banack and Cox, 2003a } \\
\text { Murch et al., 2004a }\end{array}$} \\
\hline & Bound: $48^{\star \star}$ & \\
\hline Leaf tissue of $C$. micronesica from Hawaii & Bound: $738^{\star \star}$ & Murch et al., 2004a \\
\hline \multirow{2}{*}{$\begin{array}{l}\text { Female gametophyte of seeds of } C \text {. circinalis from Guam } \\
(n=6)\end{array}$} & Free: $750 \pm 110$ & \multirow[t]{2}{*}{ Duncan et al., 1989} \\
\hline & Free: $1220 \pm 20 a$ & \\
\hline $\begin{array}{l}\text { Female gametophyte of seeds of } C \text {. rumphii from Yigo, } \\
\text { Guam }(n=6)\end{array}$ & Free: $1250 \pm 100$ & Duncan et al., 1989 \\
\hline $\begin{array}{l}\text { Female gametophyte of seeds of } C \text {. rumphii from Merizo, } \\
\text { Guam }(n=6)\end{array}$ & Free: $1390 \pm 90$ & Duncan et al., 1989 \\
\hline $\begin{array}{l}\text { Female gametophyte of seeds of } C \text {. rumphii from Florida } \\
(\mathrm{n}=2)\end{array}$ & Free: $1560 \pm 170$ & Duncan et al., 1989 \\
\hline $\begin{array}{l}\text { Female gametophyte of seeds of } C \text {. media from Florida } \\
(n=1)\end{array}$ & Free: $290 \pm 10$ & Duncan et al., 1989 \\
\hline $\begin{array}{l}\text { Female gametophyte of seeds of } C \text {. revoluta from Florida } \\
(n=1)\end{array}$ & Free: $340 \pm 30$ & Duncan et al., 1989 \\
\hline Seeds of $C$. circinalis & $\begin{array}{l}\text { Free: } 171 \# \\
\text { Total: } 244 \#\end{array}$ & Dossaji and Bell, 1973 \\
\hline Leaves of C. circinalis & $\begin{array}{l}\text { Free: } 283 \# \\
\text { Total: } 283 \#\end{array}$ & Dossaji and Bell, 1973 \\
\hline Seeds of C. revoluta & $\begin{array}{l}\text { Free: } 218 \# \\
\text { Total: } 286 \#\end{array}$ & Dossaji and Bell, 1973 \\
\hline Leaves of $C$. revoluta & $\begin{array}{l}\text { Free: } 248 \# \\
\text { Total: } 260 \#\end{array}$ & Dossaji and Bell, 1973 \\
\hline Seeds of C. media & $\begin{array}{l}\text { Free: } 21 \# \\
\text { Total: } 21 \#\end{array}$ & Dossaji and Bell, 1973 \\
\hline Leaves of C. media & $\begin{array}{l}\text { Free: } 24 \# \\
\text { Total: } 24 \#\end{array}$ & Dossaji and Bell, 1973 \\
\hline Leaves of C. neocaladonica & $\begin{array}{l}\text { Free: } 126 \# \\
\text { Total: } 126 \#\end{array}$ & Dossaji and Bell, 1973 \\
\hline
\end{tabular}


Table 3 cont. Amount of free, protein-bound and total BMAA in cycad plants of the genus Cycas used for flour production by the Chamorro people. For species identification, see footnote in Chapter 2.

\begin{tabular}{lll}
\hline Product (number of samples) & BMAA content (mg/kg dry weight) & Reference \\
\hline Seeds of C. thuarsii & $\begin{array}{l}\text { Free: } 83 \# \\
\text { Total: } 93 \#\end{array}$ & Dossaji and Bell, 1973 \\
Leaves of C. thuarsii & Free: $96 \#$ & \\
& Total: $109 \#$ & Dossaji and Bell, 1973 \\
Leaves of C. rumphii & Free: $120 \#$ & \\
& Total: $132 \#$ & Dossaji and Bell, 1973 \\
Seeds of C. cairnsiana & Free: $24 \#$ & \\
& Total: $24 \#$ & Dossaji and Bell, 1973 \\
Leaves of C. cairnsiana & Free: $24 \#$ & \\
& Total: $24 \#$ & Dossaji and Bell, 1973 \\
Leaves of C. siamensis & Free: $112 \#$ & \\
& Total: $113 \#$ & Dossaji and Bell, 1973 \\
Leaves of C. pectinata & Free: $112 \#$ & Dossaji and Bell, 1973 \\
& Total: $116 \#$ & \\
\hline
\end{tabular}

* material collect in Guam;

**material collect from a botanical garden in Hawaii;

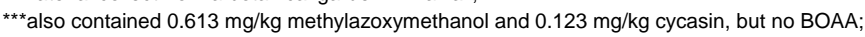

\# recalculated to fresh weight;

amorphologically deviating seeds not unambiguously identified collected 1 month before harvest of the other seeds in the

study (and stored frozen)

Table 4. The occurrence of free BMAA in leaf tissue from various cycad species (Duncan, 1991).

\begin{tabular}{|c|c|}
\hline Species & BMAA content (mg/kg dry weight) \\
\hline Bowenia serrulata $(n=3)$ & $<2.9$ \\
\hline Bowenia spectabilis $(n=3)$ & 27.1-35.4 \\
\hline Cycas cairnsiana $(n=2)$ & $117-123$ \\
\hline Cycas circinalis (Guam) & 1314 \\
\hline Cycas circinalis (young, $n=3$ ) & $1580-1830$ \\
\hline Cycas circinalis (mature, $\mathrm{n}=3$ ) & $1480-1580$ \\
\hline Cycas kennedyana $(n=2)$ & $69.2-72.2$ \\
\hline Cycas wadel $(n=2)$ & $11.6-16.0$ \\
\hline Cycas media $(n=2)$ & $316-346$ \\
\hline Cycas siamensis $(n=2)$ & $5.0-11.0$ \\
\hline Dioon edule $(n=6)$ & $7.81-15.7$ \\
\hline Lepidozamia hopei $(n=2)$ & $3.7-5.6$ \\
\hline Lepidozamia peroffshyana $(n=2)$ & $20.8-31.2$ \\
\hline Macrozamia communis $(n=2)$ & $6.5-11.1$ \\
\hline Macrozamia diplomera $(n=2)$ & $9.8-12.4$ \\
\hline Macrozamia heteromera $(n=2)$ & $17.2-18.0$ \\
\hline Macrozamia lucida $(n=2)$ & $7.9-9.1$ \\
\hline Macrozamia lucida $(n=3)$ & $14.7-18.0$ \\
\hline Macrozamia macdonnelli $(n=2)$ & $26.2-28.0$ \\
\hline Macrozamia miquelli $(n=6)$ & $5.86-12.0$ \\
\hline Macrozamia moorei $(n=2)$ & $2.9-5.8$ \\
\hline Macrozamia pauli guillelmi $(n=3)$ & $37.7-41-6$ \\
\hline Macrozamia riedlei $(n=2)$ & n.d. \\
\hline Macrozamia spiralis $(n=3)$ & $14.6-22.4$ \\
\hline Macrizamia stenomera $(n=2)$ & 18.3-23.6 \\
\hline
\end{tabular}


Because BMAA toxicity in primates requires administration of daily doses of more than $100 \mathrm{mg} / \mathrm{kg}$ over a period of 3 months, Duncan (1991) concluded that if BMAA is the agent responsible for Zamia poisoning (intoxications by feeding on Macrozamia plants) in cattle and sheep, these animals must be remarkably sensitive to the neurotoxic effects of the small amounts of BMAA found in cycad leaves. If the observed levels of free BMAA are good markers for the total level of BMAA, the chemical analytical data shown in Table 4 on cycad leaf material indicate that large doses of BMAA are unlikely to arise from consumption of cycads other than those of the genus Cycas. Therefore, it might be another toxic principle that is responsible for livestock poisoning.

\subsection{Cycad flour}

Traditional Chamorro foods include tortillas, dumplings, and cakes that up to the 1950's was prepared from flour of Cycas circinalis seeds (Kisby et al., 1992; Banack and Cox, 2006). ${ }^{4}$ When preparing the flour, the relatively large cycad seed sclerotesta is cracked, the sarcotesta (kashkada) removed, and the gametophyte (piptega) cut into slices. The slices are soaked in water for one week with repeated changes of water until the smell of urine disappear. This is a sign that the toxic principle has been washed away. It is well known among Chamorros that unwashed gametophytes and poorly 'cleaned' flour are toxic. There are anecdotal reports of illness following ingestion of cycad flour products (Whiting, 1963). In an early ethnographic report it is stated that a chicken, goat, sheep, pig or cat may be allowed to drink from one of the later changes of water and inspected for its well being to secure that no toxin remains and that the seed material is safe to be used (Luis de Ibáňez, 1871). When the seed sliced were found safe, they were dried and grated into a fine powder. Because of this traditional use of a potentially toxic material, it was already in the $1900^{\text {th }}$ century speculated that the cycad flour could be responsisble for the endemic neurological illnesses among the Chamorros, which at that time had not received any diagnosis.

The Chamorro way to prepare cycad tortillas and crab dumplings are as follows (Banack et al., 2006). The flour is mixed with water, kneaded into a soft dough, and rolled into a round flat platter. Two types of tortillas can be made - "thick and thin" - thin tortillas cooking faster in small amounts of oil. Cycad flour dumplings are initially prepared as the tortillas but smaller flat pieces of dough are filled with meat from land crabs, supplied coconut cream from grated endosperm of Cocos nucifera fruits,

\footnotetext{
4 The starchy granules made from coarse cycad flour is sometimes referred to as "cycad sago". This is not to be confused with sago as a major food item obtained mainly from a palm of the genus Metroxylon.
} 
and seasoned with salt and black pepper before being deep-fried in oil. A thickened broth or soup can also be made from cycad flour.

Because of the recognised risk of using cycad flours, and cereal flours becoming easily available from the shops of American settlers on the island, the role of cycad flour in traditional Chamorro food has diminished since the 1950's.

In the studies on cycad flour presented in Table 5, the analysed material was taken from residents of several villages on Guam and from the village of Songsong on the neighbouring island of Rota.

Table 5. Amount of free, protein-bound and total BMAA in cycad seed flour prepared traditionally by the Chamorro people of Guam.

\begin{tabular}{|c|c|c|}
\hline Product (number of samples) & $\begin{array}{l}\text { BMAA content }(\mathrm{mg} / \mathrm{kg} \text { dry } \\
\text { weight); } F=\text { free form; } B=\text { bound } \\
\text { form; } T=\text { total }\end{array}$ & Reference \\
\hline $\begin{array}{l}\text { Unprocessed flour from seeds of } C \text {. circinalis } \\
\text { collected in various regions of Guam }(n=4)\end{array}$ & Free: $750-1330$ & Duncan et al., 1988; Duncan, 1991 \\
\hline $\begin{array}{l}\text { Flour prepared from C. circinalis and washed a } \\
\text { single time }\end{array}$ & Free: 126.1 & Duncan, 1991 \\
\hline \multirow[t]{4}{*}{ Cycad flour from Yigo, northern Guam } & Free: n.d. & Banack and Cox, 2003a \\
\hline & Free: 8 (n.d.-11.8) & Duncan et al., 1990 \\
\hline & Free: $5(0.3-9.5)$ & Kisby et al., 1992 \\
\hline & Bound: $39.8-49.0$ & Murch et al., 2004a \\
\hline $\begin{array}{l}\text { Cycad flour from central Guam (Tumon, Sinajana, } \\
\text { Mongmong, Yona) }(n=6)\end{array}$ & Free: 34 (5.7-97.0) & Duncan, 1991 \\
\hline \multirow{2}{*}{$\begin{array}{l}\text { Cycad flour from southern Guam (Inarajan, } \\
\text { Talofoto, Merizo, Umatac, Agat) }(n=7)\end{array}$} & Free: 58 (n.d.-146) & Duncan, 1991 \\
\hline & Free: 5.5 & \\
\hline$(n=2)$ & Free: 7 (n.d.-18.4) & Banack and Cox, 2003 a \\
\hline \multirow[t]{2}{*}{$(n=7)$} & & Kisby et al., 1992 \\
\hline & Bound: 118 (92-169) & \\
\hline$(n=3)$ & & Murch et al., $2004 a$ \\
\hline Cycad flour, Rota $(n=1)$ & Free: n.d. & Duncan, 1991 \\
\hline$(n=2)$ & Free: 8 (n.d.-16.3) & Kisby et al., 1992 \\
\hline Cycad flour, whole Guam $(n=12)$ & $\begin{array}{l}\text { Free: } 26.6 \pm 33.4 \\
(1.5-78.1)\end{array}$ & Duncan et al., 1990 \\
\hline \multirow[t]{2}{*}{ Cycad flour, mixture from various regions } & $0.003^{\star}$ & Wilson et al., 2002 \\
\hline & $0.001^{\star *}$ & \\
\hline Traditional wheat flour & Free: n.d. & Duncan, 1991 \\
\hline
\end{tabular}

*also contained $0.144 \mathrm{mg} / \mathrm{kg}$ BOAA, but no methylazoxymethanol or cycasin; **also contained $0.613 \mathrm{mg} / \mathrm{kg}$ methylazoxymethanol and $0.123 \mathrm{mg} / \mathrm{kg}$ cycasin, but no BOAA;

Preparation of the flour varied considerably between families but always involved a series of water washes. However, the intensity of washing varied between families. Washed flakes were dried and at a suitable time ground to a flour. The time between the actual preparation of the flour and the time when it was analysed varied between the samples.

Analyses of seeds of various age indicate that BMAA is fairly stable, as levels do not differ much. Duncan et al. (1989) analysed cycad flour prepared the Guamian way and stored dry at room temperature for ten years and found a BMAA level of around $40 \%$ of that normally found in fresh flour. Although this indicates that the BMAA content of cycad flour 
is quite stable during storage, the level of BMAA in the analysed flour when it was fresh is not known (Duncan et al., 1988, 1989).

The most extensive studies on BMAA-content of cycad flour have been published by Duncan et al. (1990) and Kisby et al. (1992). The first of these investigators analysed around a dozen of flour samples prepared by a Chamorro women from C. circinalis seeds collected from Northern Guam (Tarague Beach) over three years. As shown in Table 5, the variation in free BMAA content of the unprocessed seeds was small, from 750 $\mathrm{mg} / \mathrm{kg}$ to $1330 \mathrm{mg} / \mathrm{kg}$ (Duncan et al., 1988). Flour that had been washed with water a single time contained $126 \mathrm{mg}$ free BMAA per $\mathrm{kg}$ flour, whereas properly processed flour (several changes of water) contained much lower levels of BMAA. Flour samples with correctly prepared flour from more than ten different villages on Guam did not reveal any geographical region where the flour contained particularly high levels of BMAA. Although a quick look on the data of Table 2 could indicate increasing levels of free BMAA in C. circinalis seeds and flours going from Northern to Southern Guam, no statistically significant difference between these regions were found. The mean level of free BMAA in the thirty flour samples analysed by Duncan and co-workers was $36 \mathrm{mg} / \mathrm{kg}$, which can be compared with $1140 \mathrm{mg} / \mathrm{kg}$ in the raw unprocessed C. circinalis female gametophyte tissue. Thus, the data confirm an extensive removal of free BMAA on washing of the seeds during flour production. For all samples, Duncan et al. (1990) noted that at least $85 \%$ of the free BMAA was removed by the water extraction of the seeds, and in more than half of the samples $99 \%$ of the free BMAA was extracted by the leaching process. Most had been removed during the first washing in water. The investigators concluded that the rather wide distribution in BMAA levels of traditional flour, most probably resulted from variations in the thoroughness of washing the flour rather than age-related or regional differences in the BMAA content of cycad seeds (Duncan et al., 1989, 1990; Duncan, 1991).

Kisby et al. (1992) analysed cycad flour from 17 Chamorro residents living in different areas of Guam. The method used by these investigators had a very short extraction period compared with the method applied by Duncan et al. (1990) - 3 minutes with a large sample as compared to $72 \mathrm{~h}$ with a small sample - and in addition a different detection method (derivatization followed by HPLC/fluorescence as compared to GC-MS). The data of Kisby et al. (1992), therefore, should be interpreted with care. These differences resulted in lower amounts of BMAA being detected: on average $5.44 \pm 6.44 \mathrm{mg} / \mathrm{kg}$ (as recovery was $54 \%$ it could be recalculated to $10,07 \pm 11.93 \mathrm{mg} / \mathrm{kg}$ ). Kisby et al. (1992) also analysed for cycasin, but found no correlation between the content of cycasin and BMAA in cycad flour $(r=0.008)$. 
The single study that analysed for bound BMAA in traditional cycad flour (Murch et al., 2004a), reports levels that are 4-18 times higher than those reported in studies only analysing for free BMAA.

In addition to the raw flour ingredient, several traditional Chamorro foods prepared with flour has been analysed for the presence of free and bound BMAA. Dough, tortillas and dumplings prepared by Chamorro people from washed cycad flour had no detectable free BMAA, but retained 4.7, 334, and $84 \mathrm{mg}$ protein-bound BMAA per $\mathrm{kg}$, respectively. (Banack et al., 2006). Other traditional Chamorro foods include wild animals such as feral deer, pigs, land crabs and flying foxes, which provide protein alternatives to fish.

\subsection{Conclusions on occurrence of BMAA in cycads}

Although BMAA has been identified in all cycad species investigated, the amount detected varies considerably between species. Cycas circinalis, which is the cycad growing in Guam and implicated as an ethiological factor for the high incidence of neurodegenerative disease on this island, contain in its seeds 750-1 $200 \mathrm{mg}$ free BMAA/kg dry weight. Some investigators claims that there in addition may be found bound BMAA. Seeds of the other two cycad species implicated in neurodegenerative disease in Japan and New Guinea (C. revoluta and C. media), only contain around $25 \%$ of the amount of BMAA in C. circinalis. Traditional Chamorro foods have been prepared using flour from seeds of $C$. circinalis. As these seeds are well known to be toxic, they are thoroughly washed during flour production to remove toxins. When prepared the levels of free BMAA has been reduced to around one or a few percent of the original levels in the seed. The variation in BMAA content between flour samples is likely to be due to different thoroughness in the washing procedure used in flour production. One investigation has reported 4-18 times higher levels of protein-bound BMAA as compared to free BMAA, but as indicated in chapter 5 , this possibility needs confirmation by other analytical methodologies. BMAA is comparatively stable during storage of the flour. 


\section{The theory of biomagnification and identification of BMAA in human tissues}

Because of the difficulty to explain a "slow toxin"-action of watersoluble free BMAA molecules on the neurological system, Murch et al. (2004a) investigated the possibility of biomagnification of BMAA and leakage from bound forms of BMAA. They found that axenic cultures of cyanobacteria, initially isolated from collaroid cycad roots, contained approximately 240 times more bound BMAA than free BMAA, 72 as compared to $0.3 \mathrm{mg} / \mathrm{kg}$ bacteria. Collaroid roots of the cycad Cycas micronesica contained $2 \mathrm{mg}$ BMAA per $\mathrm{kg}$ root in bound form. As noncollaroid roots had no symbiosis with cyanobacteria and contain no BMAA, it seems as though BMAA has to leak out from the cyanobacteria and be taken up by the collaroid cycad roots. When other cycad tissues were investigated the results pointed to that leaf tissue contained $738 \mathrm{mg}$ BMAA per kg tissue, outer seed layers 48 mg BMAA per kg tissue, the sarcotesta $89 \mathrm{mg}$ per $\mathrm{kg}$ tissue, and the female gametophyte $81 \mathrm{mg}$ BMAA per $\mathrm{kg}$ tissue. These observations do not only suggest that the total BMAA exposure may be higher than that indicated by the amount of free BMAA, but also that the amount of BMAA found may increase going from cyanobacteria to cycads.

\subsection{Flying foxes}

Various parts of the cycad tree is consumed by higher organisms. There are 60 species of flying foxes. Historically, Guam was home to two species, Pteropus tokudae Tate and P. marinannus Desmarest (Tate, 1934). However, P. tokudae has not been observed since 1968 and is now declared extinct by the World Conservation Union, whereas $P$. mariannus is considered endangered by the same organisation. Most likely due to the introduced brown tree snake, no young $P$. mariannus has survived to adulthood since 1982 (Wiles et al., 1995). The diet of $P$. mariannus consists of 67 plant species identified from 35 plant families, most of which are from the primary forest. Cycad seeds are among its favorite 10 food items (Wiles, 1987). Flying foxes are, therefore, expected to be exposed both to free and protein-bound BMAA.

Cox and co-workers have in three studies presented data on the occurrence of BMAA in museum samples of these animals (Banack and Cox, 
2003a, Murch et al., 2004a; Banack et al., 2006). These studies have been summarized in Table 5. There is no confirmation from other laboratories that flying foxes from Guam contain BMAA. In the first of the three studies, dried skin from three samples of Pteropus mariannus mariannus were reported to contain on average $3356 \mathrm{mg}$ free BMAA per $\mathrm{kg}$ tissue (Banack and Cox, 2003a). The second publication reported on more then ten times lower levels of protein-bound BMAA in hair $(146 \mathrm{mg} / \mathrm{kg})$ and wing membrane $(2 \mathrm{mg} / \mathrm{kg}$ ) (Murch et al., 2004a). The most extensive study on the occurrence of BMAA in flying foxes is the latest of the studies (Banack et al., 2006). This study was based on 21 accessioned flying fox specimens on deposit in various museums, and represented two subspecies of Pteropus mariannus (Pteropus mariannus mariannus, from Guam, $\mathrm{n}=9$; Pteropus mariannus yapensis, from Yap, $\mathrm{n}=8$ ), and a closely related species, Pteropus tonganus Quoy and Gaimard, from Samoa $(n=4)$. Substantial differences in BMAA concentrations between individual flying foxes from the same island were noted and may reflect individual foraging preferences. The specimens were preserved either as dried skins, fluid-preserved samples (formalin fixed and stored in $70 \%$ alcohol), or frozen carcasses. The study showed that both free and proteinbound BMAA can be found in tissues of Guamanian flying foxes (Pteropus mariannus mariannus), including internal organs, muscles, skin and hair. On average the levels of protein-bound BMAA were four times higher than the level of free BMAA. In frozen material the levels of BMAA varied substantially between tissues, whereas no significant difference in BMAA level between tissues were found in fluid-preserved specimens. The authors speculated in the possibility that proteolytic enzymes (frozen specimen) or long-term protein degradation (dried specimen) could result in release, over time, of free BMAA from the protein fraction. They also suggested that in the liquid-preserved material diffusion of BMAA molecules in the liquid could have occurred over the decades they had been stored. No free BMAA was detected in any of the Pteropus tonganus samples. Two analyses for protein-bound BMAA performed on one of these samples revealed BMAA from the outer surface (hair and skin). When the interior wing sample from one of these fluid-preserved samples were analysed no BMAA could be found. The authors suggested that the presence of BMAA on the outer hair and skin was due to contamination, perhaps from 20 years of storage of the tissues in large fluid filled vats that may, at one time, have contained multiple specimens. Thus, it can be concluded that a large variation in the level of free and bound BMAA has been reported in tissue samples of flying foxes, and, at present, this variation cannot be explained. 
Table 6. Amount of free, protein-bound and total BMAA in museum samples of flying foxes from Guam and nearby places (Banack and Cox, 2003a *; Murch et al., 2004a**; Banack et al., 2006). If not stated otherwise samples were preserved in fluid.

\begin{tabular}{|c|c|}
\hline Product (number of samples) & BMAA content ( $\mathrm{mg} / \mathrm{kg}$ dry weight) \\
\hline Pteropus mariannus, dried skin (50 y.o.) ( $n=3$ ) & $1237-7502^{*}$ \\
\hline Pteropus mariannus mariannus, dried skin $(n=3)$ & $\begin{array}{l}\text { Free: } 123 \pm 102 \\
\text { Bound: } 479 \pm 689\end{array}$ \\
\hline Pteropus mariannus mariannus, skin $(n=6)$ & $\begin{array}{l}\text { Free: } 133 \pm 196 \\
\text { Bound: } 88 \pm 78 \\
\text { Total: } 233 \pm 184(78-552)\end{array}$ \\
\hline $\begin{array}{l}\text { Pteropus mariannus mariannus, hair from } 52 \text { y.o dried sample } \\
(n=1)\end{array}$ & $\begin{array}{l}\text { Bound: } 146^{\star *} \\
\text { Bound: } 1859\end{array}$ \\
\hline \multicolumn{2}{|l|}{ Undefined sample $(n=1)$} \\
\hline Pteropus mariannus mariannus, wing $(n=2)$ & $\begin{array}{l}\text { Free: } 126 \pm 97 \\
\text { Bound: } 359 \pm 453\end{array}$ \\
\hline Pteropus mariannus mariannus, hair $(n=6)$ & $\begin{array}{l}\text { Bound: } 305 \pm 421 \\
\text { Total: } 380 \pm 411(136-1202)\end{array}$ \\
\hline Pteropus mariannus mariannus, dried wing membrane (52 y.o) & Bound: $2^{* *}$ \\
\hline Pteropus mariannus mariannus, skull muscle $(n=1)$ & $\begin{array}{l}\text { Free: } 63 \\
\text { Bound: } 13\end{array}$ \\
\hline Pteropus mariannus mariannus, brain $(n=2)$ & $\begin{array}{l}\text { Free: } 19 \pm 13 \\
\text { Bound: } 78 \pm 80\end{array}$ \\
\hline Pteropus mariannus mariannus, intestine $(n=2)$ & $\begin{array}{l}\text { Free: } 30 \pm 23 \\
\text { Bound: } 77 \pm 72\end{array}$ \\
\hline Pteropus mariannus mariannus, kidney $(n=6 / 5)$ & $\begin{array}{l}\text { Free: } 29 \pm 14 \\
\text { Bound: } 75 \pm 92 \\
\text { Total: } 102 \pm 89(29-242)\end{array}$ \\
\hline Pteropus mariannus mariannus, liver $(n=6)$ & $\begin{array}{l}\text { Free: } 46 \pm 6 \\
\text { Bound: } 197 \pm 304 \\
\text { Total: } 241 \pm 302(51-837)\end{array}$ \\
\hline Pteropus mariannus mariannus, chest muscle $(n=4 / 6)$ & $\begin{array}{l}\text { Free: } 59 \pm 12 \\
\text { Bound: } 319 \pm 238 \\
\text { Total: } 326 \pm 222(75-710)\end{array}$ \\
\hline Pteropus mariannus mariannus, shoulder muscle $(n=2)$ & $\begin{array}{l}\text { Free: } 74 \pm 47 \\
\text { Bound: } 174 \pm 134\end{array}$ \\
\hline Pteropus mariannus yapensis, dried skin $(n=3)$ & $\begin{array}{l}\text { Free: } 272 \pm 43 \\
\text { Bound: } 238 \pm 376\end{array}$ \\
\hline Pteropus mariannus yapensis, skin $(n=2)$ & Total: $600 \pm 435(292-907)$ \\
\hline Pteropus mariannus yapensis, dried hair $(n=2)$ & Bound: $338 \pm 170$ \\
\hline Pteropus mariannus yapensis, hair $(n=3)$ & Total: $907 \pm 816$ (430-1849) \\
\hline Pteropus mariannus yapensis, kidney $(n=1)$ & $\begin{array}{l}\text { Free: n.d. } \\
\text { Bound: n.d. }\end{array}$ \\
\hline Pteropus mariannus yapensis, frozen liver $(n=3)$ & $\begin{array}{l}\text { Free: } 232 \pm 329 \\
\text { Bound: } 323 \pm 437 \\
\text { Total: } 699 \pm 180(572-826)\end{array}$ \\
\hline Pteropus mariannus yapensis, liver $(n=2 / 1)$ & $\begin{array}{l}\text { Free: } 109 \pm 53 \\
\text { Bound: } 58\end{array}$ \\
\hline Pteropus mariannus yapensis, muscle $(n=1)$ & Free: n.d. \\
\hline Pteropus tonganus, dried hair and skin $(n=2)$ & Free: n.d. \\
\hline Pteropus tonganus, hair and skin $(n=2)$ & $\begin{array}{l}\text { Free: n.d. } \\
\text { Bound: } 727 \pm 967\end{array}$ \\
\hline Pteropus tonganus, wing $(n=1)$ & Free: n.d. \\
\hline
\end{tabular}


In earlier times, when it still was allowed to hunt these animals and they still were so common that they could be found on Guam, they were highly desirable food items served at Chamorro weddings, village fiestas, and religious events (Wiles and Payne, 1986; Lemke, 1992). The traditional way to prepare flying foxes has been described by Banack et al. (2006). Two flying foxes carcases were washed in fresh water, cut into six pieces, and placed in a large pot half filled with water (around 7 litre). The pot was placed on open fire and boiled for 25 minutes with the lid on. Three cans of whole kernel maize were added along with salt, black pepper, and monosodium glutamate (3 tablespoons). Half an hour later, two coconuts were frated and 1 litre of water added. Coconut milk was added to the stew, which was then removed from the fire and allowed to cool for 5 minutes before being consumed. After cooking, the entire animal is consumed, including brains, viscera, fur, and wing membranes (Wiles and Payne, 1986; Lujan, 1992). The typical adult serving was around 1 litre. Both free and protein-bound BMAA was found in the broth of flying fox stew, the amounts being 0.42 and $12.34 \mathrm{mg}$ per litre, respectively (Banack and Cox, 2006).

In another study the same researchers investigated the influence of cooking on the level of free and bound BMAA in various organs of flying foxes (Banack and Cox, 2006). Before preparation of the frozen flying fox free and bound BMAA was found in the heart, kidney, liver, spleen, muscle, skin, intestine, and wing. After cooking bound BMAA could still be found in all tissues except the skin. The level was always lower, except for in the intestine, where the level was increased. After cooking, free BMAA could no longer be found in the heart, kidney, and skin. The level in the liver was reduced, but levels in the muscle increased. Thus, cooking generally reduced the levels of BMAA in flying fox tissue.

Interestingly, the rise and fall in consumption of Guam flying foxes is shadowed by a rise and fall of the incidence of ALS-PDC in Guam (Fig. 1) (Garruto et al., 1986; Haddock and Santos, 1992). It should be pointed out, however, that the data used to assess the availability of flying foxes is poor indeed and, therefore, results in very shaky consumption data.

There is a single analysis of animal material besides the flying foxes. Banack et al. (2006) analysed hair from a Philippine deer (Cervus mariannus) that is known to ingest cycad seeds, and showed that the material contained $3 \mathrm{mg}$ bound BMAA per $\mathrm{kg}$. The hair was not analysed for free BMAA. 


\subsection{Brain material and other tissues from control individuals and patients with degenerative neurological disease}

The hypothesis linking the diet of the Chamorro people of Guam to the increased incidence of the neurodegenerative disease ALS-PDC observed in this population have been discussed over the last fifty years. The hypothesis was recently revitalized when the suspected inducing agent BMAA was suggested to be biomagnified in the food chain. Flying foxes consume large amounts of cycad seeds and the Chamorro people not only consume flour of cycad seeds but also flying foxes, resulting in relatively high exposure to BMAA and a potential of the compound being transferred to humans (Cox et al., 2003).

A few years ago, an American research group (Cox et al., 2003; Murch et al., 2004b) found free BMAA in human brain tissue. In the initial study on ALS-PDC patients, Cox and co-workers found a mean of $6 \mathrm{mg}$ free BMAA per $\mathrm{kg}$ brain tissue from the frontal cortex of six Chamorro patients who died from ALS-PDC. However, they also found a mean of $6.6 \mathrm{mg}$ free BMAA per $\mathrm{kg}$ brain tissue from the frontal cortex of two patients from Canada who died from Alzheimer's disease. No BMAA was found in brain tissues from a group of 13 individuals who died of causes unrelated to neurodegenerative disease (Cox et al., 2003). Why deposition of BMAA may occur in specific tissues has not been explained.

The same laboratory extended their studies on human brains by analysing for both free and protein-bound BMAA in gyrus tissue from diseased Chamorro patients clinically diagnosed with ALS-PDC, two Chamorro individuals who died of other causes than neurological illness, and a control group of 15 Canadians (2 patients with Alzheimers disease and 13 patients who did not die of neurological illness) (Murch et al., 2004b). Free BMAA was found in material from the superior frontal gyrus tissue of five of the six Chamorro patients with ALS-PDC (3.3-10.1 $\mathrm{mg} / \mathrm{kg}$ ) and protein-bound BMAA in all ALS-PDC patients (82-1190 $\mathrm{mg} / \mathrm{kg}$ ). Bound BMAA was also found in one of the asymptomatic Chamorro controls with no observable histological abnormalities. BMAA was 60 - to 130 -fold more common in the bound form than in the free amino acid pool.

The finding of BMAA occurring in Canadian Alzheimer patients stimulated further studies on the frontal cortex, temporal cortex, parahippocampal gyrus, caudate, and cerebellum from seven patients with Alzheimer's disease and one patient who did not die of a neurological illness (Murch et al., 2004a). One patient had $45.7 \mathrm{mg}$ BMAA per $\mathrm{kg}$ frontal cortex, and 29.6 mg BMAA per kg caudate but no BMAA in other parts of the brain. One patient had $34.2 \mathrm{mg}$ bound BMAA per kg parahippocampal gyrus but noting in other parts. The third patient had $53.1 \mathrm{mg}$ 
bound BMAA in the temporal cortex and nothing in other parts, a fourth patient $33.7 \mathrm{mg}$ bound BMAA in the temporal cortex and $41.2 \mathrm{mg}$ bound BMAA in frontal cortex but nothing in other tissues, a fifth $25.9 \mathrm{mg}$ bound BMAA in the frontal cortex and nothing in other brain tissues, and the last patient $170.8 \mathrm{mg}$ bound BMAA in temporal cortex, $235.6 \mathrm{mg}$ in parahippocampal gyrus, and $185.5 \mathrm{mg}$ in caudate, but noting in other brain parts. Thus, the only part of the brain were bound BMAA was never identified in the Alzheimer patients was the cerebellum.

As similar brain tissue levels to those found in Chamorro ALS-PDC patients were also found in patients with Alzheimers disease that were assumed never to have consumed BMAA-containing foods but not in control individuals, and there were indications that BMAA is produced by cyanobacteria, the authors commented that the presence of BMAA in the Canadian patients opens the possibility that cyanobacterial neurotoxins are biomagnified in different ways in various ecosystems. If BMAA is the cause of Altzheimer's disease through contamination of drinking water through leakage from cyanobacteria, a variation in brain levels of BMAA would be expected in the normal population. But Cox et al. (2003) only found BMAA in Alzheimer patients. If BMAA occurs in brain tissue of patients with neurodegenerative disease at all, might it be a consequence of the disease and not the cause of the disease?

The findings of BMAA in brain material that has been stored in formaldehyde, and the possibility that these findings of BMAA may be due to artefacts due to methylation of amino groups during storage is an issue of interest.

In trying to confirm the observation of Cox and co-workers, Montine et al. (2005) assayed for free BMAA in the middle frontal gyrus of brains of five control subjects and five Caucasian patients with Alzheimer's disease from the United States Pacific Northwest, as well as in Chamorro brains from patients with and without Parkinson-dementia complex. In contrast to Cox and co-workers, Montine et al. (2005) detected no free BMAA in any of these samples. The latter investigators used no fixative or preservative with any of the tissues used for BMAA analysis. Instead they used frozen material. Tissue homogenisation, precolumn derivatization with 9-fluorenylmethylchloroformate, and high-performance liquid chromatography separation with fluorescence detection were performed as described by others (Kisby et al., 1988). The limit of detection was 1.0 pmol of BMAA ( $0.15 \mathrm{ng})$, similar to the 0.6 pmol reported by Kisby et al. (1988), but much lower than the 100 pmol reported by Murch et al. (2004b). Spiking with $1 \mathrm{ng}$ of BMAA prior to derivatization with fluorenylmethylchloroformate easily allowed detection of BMAA. The one systematic difference between the study of Montine et al. (2005) and all studies by Cox and co-workers was that the former used the standard tissue preparation protocol for neurochemical research (flash-frozen tissue with fixative or preservative), whereas Cox and co-workers used tis- 
sues that had been fixed in paraformaldehyde and preserved in $15 \%$ sucrose for years (Cox et al., 2003; Murch et al., 2004a; 2004b). Montine et al. (2005) concluded that high concentraions of free BMAA in brain neither is a common feature of individuals in the United States Pacific Northwest, nor in Chamorros on Guam, regardless of the presence of Alzheimer's disease or Parkinson-dementia complex. Montine et al. (2005) speculated that these contrasting results may be due to differences in the methods of tissue fixation which may lead to artefacts.

Commenting further on this statement, Montine et al. (2005) concludes that

\footnotetext{
"these findings would have wide public health implications because bioaccumulation of cyanobacterial BMAA may occur through different sources in food chains far from Guam. However, undetectable BMAA in controls implies not only an environmental source of BMAA but also selective accumulation of this molecule in the brain of patients with Alzheimer's disease".
}

\subsection{Conclusion on the theory of biomagnification of BMAA and identification of BMAA in human tissues}

Biomagnification of BMAA has been suggested to be a mechanism that makes it possible for humans to be exposed to BMAA levels that would have implications for neurological toxicity, as it was early recognised that the levels of BMAA in cycad flour was to low to explain the local high incidence of neurodegenerative disease on Guam. The theory of biomagnification was launched after BMAA was reported to occur at more than 200 times higher level in bound form in cyanobacteria than in free form (see Chapter 8). The compound was believed to be transferred from cyanobacteria, which in the collaroid roots live in symbiosis with the cycad tree. Although only low levels of BMAA are found in these roots, higher levels are found in other tissues of the tree. Flying foxes consume large amounts of cycad seeds and the Chamorro people not only consume flour of cycad seeds but also flying foxes, thereby being exposed to relatively high amounts of BMAA.

However, as thoroughly described in Chapter 5 , there is very little scientific proof that BMAA can be found at any signicant level in cyanobacteria - either in free or in bound form. As the report that BMAA occurs at high levels in flying foxes may be questioned, the whole theory of biomagnification of BMAA is questioned.

The same scepticism prevail in relation to the information that BMAA has been detected in human brain (mainly in bound form) by some investigators, and not only in brains of Chamorro people diagnosed with neurodegenerative disease but also in control altzheimer patients that have never consumed cycad flour or flying foxes. It was suggested that these patients had been exposed to BMAA via drinking water from surface 
waters that were contaminated by BMAA-producing cyanobacteria. Montine et al. (2005) speculated that the contradictory findings reported in stored brain material may be due to differences in the methods of tissue fixation, which may lead to artefactual levels of what appears to be BMAA. 


\section{Is BMAA produced by cyanobacteria}

In 2003 Cox and coworkers found BMAA to be present in cyanobacteria of the strain Nostoc, which live in symbiosis with the coralloid roots of the cycad (Cox et al, 2003). Pursuing this line of research they continued to analyse presence of BMAA in 30 different species or strains of freeliving cyanobacteria and concluded that BMAA was present in free form in 27 of these species or strains in amounts varying from $3-6478$ mi$\mathrm{crog} / \mathrm{g}$ dry weight, and in bound form in 24 species or strains in amounts varying from 4- $5415 \mathrm{microg} / \mathrm{g}$ dry weight (Cox et al, 2005). According to Cox (2005) the presence of BMAA in cycads could be explained by the symbiosis these plants have with cyanobacteria. It was proposed that BMAA is produced by cyanobacteria infecting the coralloid roots of cycad trees as symbionts. BMAA is then transferred to the cycad where higher concentrations can be reached. The accumulation when going from one trophic level to another could be 100-fold. The biomagnification of BMAA through the chain cyanobacteria - cycads - flying foxes humans could be the explanation for the high incidence of ALS-PDC on Guam and neighboring islands, according to Cox et al. (2005).

If this theory is be confirmed, the implications for human health may be great. Cyanobacteria are abundant and ubiquitos. Cyanobacteria are found worldwide, in sweet, brackish and marine waters as well as in symbiosis with other organisms. Different species and strains thrive in all climates. The potential for humans to be exposed to cyanobacterial BMAA would be implicit. Possible exposure routes could be through drinking water or fish and seafoods that are used as foods, as well as through ingesting vegetables watered with lake-water containing cyanobacteria or plants that have cyanobacterial symbionts. Dermal exposure through bathing or inhalation of water spray whilst boating or pursuing water sports would be other possible routes of exposure.

If BMAA is produced by cyanobacteria, people who eat these organisms as health food (fresh or freeze-dried) may potentially be an exposure group at risk.

If BMAA is confirmed to be produced by cyanobacteria, analysis of drinking water taken from sources where cyanobacterial blooms occur and on fish and seafood are warranted.

However, it must be stressed that so far the findings of BMAA in cyanobacteria have not been confirmed. The present authors are among those that have been unable to identify BMAA in cyanobacteria (see section 5-7). A critical review of analytical methods used by different re- 
search groups to analyse cyanobacteria is found in section 5.5. The method used by the authors, and the results of analysis that have been carried out are discussed in sections 5.6 and 5.7.

\section{Conclusion on possible production of BMAA by cyanobacteria}

Based on the most recent analytical studies of cyanobacteria it seems unlikely that these organisms are producing BMAA in substantial amounts. As a consequence, $i$ is still an unsubstantiated hypothesis that the BMAA in cycads is produced by symbiotic cyanobacteria and not endogenously formed in the plant. 


\section{Conclusions}

A review of information available in published literature supports a true connection between cycad exposure and the development of ALS-PDC on the island of Guam. However, it is not clear which the compound(s) in the cycad is that is responsible for the development of neurological disease.

One compound that is found in cycad is $\beta$-methylaminoalanine (BMAA). Several investigators, using different methods of analysis, confirm the finding of BMAA in cycads. However, analytical methods for quantification of BMAA have not been adequately validated. This situation makes it difficult to establish or repudiate a relationship between BMAA exposure and neurological disease.

BMAA has been found experimentally to have acute toxic effects on the brain, but only at very high concentrations.

Nevertheless, human epidemiological data as well as experiences from animal feeding studies implies that the amount of BMAA available in processed cycad flour is not enough to be the main cause of degenerative neurological disease after consumption of cycad flour.

The hypothesis that BMAA, in addition to occurring in free form, also may occur in a bound form in the protein fraction, needs confirmation, and the potential binding needs to be properly defined and explained.

The possibility that BMAA in biological samples may occur as a carbamate, or as a carbamate glucuronide or as a similar compound, may potentially influence analytical findings and physiological effects. This needs to be elucidated.

The possiblility that formaldehyde has a potential to methylate amino groups of biological materials should be clarified, since this might influence results of analysis of BMAA in tissue samples stored in formaldehyde.

The statements that BMAA is widely occuring in cyanobacteria at levels that would present a human risk factor for development of neurological diseases is questioned by recent analytical studies. Therefore, also an extensive and worldwide exposure of human beings to BMAA from cyanobacteria and the associated human health risks are questioned. At the present state of knowledge, no requirements for immediate regulatory measures can be identified.

A few investigators have reported the occurrence of the toxic amino acid BOAA in cycad. Other toxic compounds have also been identified in cycad flour. It is of great interest to clarify the possible relevance of these compounds in cycad for the development of neurological diseases in humans. These naturally occurring compounds may also be present in other 
plant species to which humans are exposed via the diet or in the form of medical preparations. 


\section{Recommendations}

To be able to draw conclusions on the possible connection between BMAA and the development of degenerative neurological disease we have the following recommendations:

Only validated methods should be used for the analysis of BMAA. The validation should include ring trials with several laboratories participating. As some investigators have identified BOAA and other potentially toxic compounds in cycad material, it might be useful to develop validated analytical methods also for these compounds.

The suggested presence of BMAA in cyanobacteria, and tissues of flying foxes and in tissues from patients with degenerative neurological disease requires confirmation by validated analytical methods having a high specificity. At least two different validated methods with high specificity should be able to detect BMAA in these samples before the findings is established.

The form in which BMAA is found in various organisms should be established. In the case the compound may occur in different forms, the contribution of the various forms to the risk profile should be established.

- The possible binding of BMAA to proteins should be studied.

- The possibility that BMAA may occur as a carbamate, or as a carbamate glucuronide, or similar, in biological material should be investigated.

The possiblility that formaldehyde has a potential to methylate amino groups of biological materials and the implication this has on analysis of tissues stored in formaldehyde, should be clarified.

Toxicokinetic experiments should be performed, e.g. by autoradiography, to note tissue distribution and possible formation of tissue reservoirs of BMAA.

The relevance of high and low short-term exposure to BMAA, BOAA and other potential toxins in the cycad and the development over long term of degenerative neurological diseases should be elucidated. 



\section{Sammanfattning}

(Summary in Swedish)

Sedan 1940-talet har man sökt orsakerna till den höga förekomsten av amyotrof lateral skleros och Parkinsondemens komplex (ALS-PDC) inom den lokala Chamorropopulationen på Guam och närliggande öar i sydvästra Stilla Havet. Även om antalet fall nu har minskat från en topp under 1950-talet (179/100 000 för män och 61/100 000 för kvinnor) till en nivå som jämfört med västvärldens incidens på 1990-talet (1, 9/100 000) enbart ligger några gånger över denna, fortsätter sökandet efter sjukdomarnas orsak. Orsaksfaktorer som undersökts och avfärdats efter vetenskaplig granskning omfattar ärftliga och miljörelaterade faktorer, prioner, infektioner och brist på mikronäringsämnen. Ingen av dessa tros ha någon avgörande betydelse. Den traditionella kosten var den enda faktor som epidemiologiska studier indentifierade vara knuten till sjukdomskomplexet. Särskilt bruket av mjöl framställt från cykadfrön, en stapelföda bland Chamorropopulationen, blev ett ämne för diskussion under 1960talet. Sedan dess har det påvisats ett tydligt samband mellan konsumtion av cykadmjöl och en hög risk att utveckla ALS-PDC på Guam.

Cykadfrön innehåller ett flertal toxiska ämnen av vilka cycasin, en levertoxisk och cancerframkallande glykosid av metylazoxymetanol (MAM), är bäst känd. Då studier på försöksdjur visat att levertoxicitet är den huvudsakliga effekten av detta ämne, har sökandet efter andra toxiska ämnen med nervskadande effekt fortgått. 1967 blev aminosyran betametylamino-L-alanin (BMAA) isolerad ur cykadfrön och en hypotes presenterades att denna aminosyra kunde vara orsaken till fördröjd neurodegenerativ sjukdom. Det har emellertid argumenterats att mängden BMAA som inmundigas vid bruket av traditionellt avgiftat cykadmjöl i kosten inte är tillräckligt hög för att förorsaka neurologiska skador. Under senare tid har BMAA återkommit i rampljuset som orsak till ALS-PDC efter det att forskare föreslagit att BMAA kan ackumuleras i näringskedjan. Hypotesen är att flygande hundar, en stor fladdermusliknande art, som brukade ingå i den traditionella Chamorrofödan åt cykadfrön och lagrade BMAA i kroppen, både som fri aminosyra och bunden till proteiner. Efter analys av formalinbevarade museiexemplar av flygande hundar rapporterades fynd av BMAA. Samma forskargrupp rappoterade även fynd av BMAA i cyanobakterier av släktet Nostoc som isolerats från cykadrötter där cyanobakterierna lever i symbios med växten. Baserat på dessa fynd framförde de förslaget att BMAA producerat av cyanobakterier överförs till den symbiotiska partnern och transporteras till olika delar av växten. 
Kort efter att denna upptäckt hade lanserats presenterade samma forskargrupp en rad uppseendeväckande upptäckter. För det första rapporterade de att BMAA kunde påvisas i hjärnan från människor som avlidit på grund av neurodegenerativa sjukdomar, men inte i hjärnan från människor som avlidit av andra orsaker. Senare rapporterade de att BMAA upptäckts i 27 av 30 analyserade cyanobakteriestammar. Eftersom cyanobakterier är allmänt förekommande och bland annat uppträder i ytvatten som används som dricksvattentäkter skulle implikationen för människans hälsa kunna vara stor.

Scenariot med en tänkbar exponering av den nordiska konsumenten för BMAA (via dricksvatten) initierade ett kemiskt analytiskt arbete för att kunna identifiera BMAA, och medförde att denna riskbedömning påbörjades.

En nyckelfråga är uppenbart behovet av pålitliga analysdata. Utan adekvata extraktionsmetoder, validerade analysmetoder och pålitlig analysutrustning är det inte möjligt att testa teorin att BMAA är orsaken till neurodegenerativ sjukdom. Tidigare upptäckter indikerar att BMAA förekommer både $\mathrm{i}$ fri och $\mathrm{i}$ bunden form $\mathrm{i}$ de material som analyserats. Denna observation måste verifieras och tas med $\mathrm{i}$ beräkningen vid design av analysmetoder. För analys av cykadmaterial har flera olika kvalitativa metoder använts medan valideringar av tillfredsställande kvalitet inte har rapporterats. Av den anledningen måste alla kvantitativa data som rör BMAA tolkas med stor försiktighet. Alla analytiska data som redovisas för BMAA i andra material än cykad är baserade på en enda metod, som bygger på derivatisering av BMAA. För att kunna dra pålitliga slutsatser om eventuell förekomst av BMAA i andra material än cykad måste dessa fynd bekräftas med andra metoder, företrädesvis metoder med hög specificitet. Nyligen genomförda analyser i Sverige ifrågasätter påståendet att BMAA är vanligt förekommande i cyanobakterier.

Från djurförsök framgår att intag av obehandlade cykadfrön leder till både akuta och kroniska toxiska effekter. Toxiciteten hos cykadfröet har länge varit känd och Chamorrofolket har sköljt sönderdelade cykadfrön i vatten för att reducera mängden toxiner när de framställt cykadmjöl. Dock har även rätt behandlade cykadprodukter, där kända toxiner (cycasin, MAM, BMAA) urlakats till låga eller omätbara mängder, givit upphov till toxiska effekter. Därför har det framförts möjligheten att hittills okända toxiner i cykad kan vara orsaken till de neurodegenerativa sjukdomar som förekommit i stor omfattning på Guam.

Flera in vitro och in vivo studier har utförts med det enskilda ämnet BMAA. Dessa studier visar att BMAA orskar toxiska effekter på hjärnan vid höga doser. Studier indikerar dock att det är osannolikt att mängden BMAA i traditionellt framställt cykadmjöl är tillräckligt för att resultera i toxiska effekter på det centrala nervsystemet och ge upphov till de neurodegenerativa sjukdomar som observerats i ursprungsbefolkningen på Guam, om inte konsumtionen varit extrem. Av denna anledning är det 
inte troligt att det är BMAA som är den enda primära orsaken till degenerativa neurologiska sjukdomar hos människa, såvida inte en okänd verkningsmekanism kan ge en högre exponering av BMAA än vad tidigare förmodats, eller att toxiciteten förmedlas med andra verkningsmekansmer än de som är kända idag.

Sammanfattningsvis kan konstateras att den information som finns tillgänglig i den vetenskapliga litteraturen stödjer ett samband mellan konsumtion av cykadmjöl och utvecklingen av ALS-PDC hos lokalbefolkningen på Guam. Det är emellertid inte fastslaget vilket det toxiska ämnet i cykad är. En kandidat är BMAA som med olika analysmetoder påvisats i cykadmaterial. Detta har bekräftats av analyser i Sverige. Olyckligtvis har ingen säker och validerad metod att kvantifiera BMAA publicerats, ett förhållande som gör det svårt både att bekräfta och motbevisa ett samband mellan BMAA exponering och neurologiska sjukdomar. I experimentella studier har man funnit att BMAA ger akuttoxiska effeker på hjärnan enbart vid mycket höga koncentrationer. Humanepidemiologiska studier och in vivo studier på försöksdjur indikerar att halten BMAA i traditionellt producerat cykadmjöl inte är tillräckligt högt för att orsaka neurodegenerativ sjukdom efter intag.

Den nyligen lancerade hypotesen att BMAA är vanligt förekommande i cyanobakterier vid nivåer som skulle kunna innebära en riskfaktor för utveckling av neurologisk sjukdom ifrågasätts starkt av nyare analytiska studier. Följaktligen ifrågasätts även en allmän och omfattande exponering av människor för BMAA via cyanobakterier i dricksvatten och därav följande hälsorisker.

Med nuvarande kunskap finns ingen anledning att $\mathrm{i}$ de nordiska länderna befara en stor, allmän exponering för BMAA, och därför inte heller anledning att vidta åtgärder inom detta område. 



\section{References}

Adiga, O. R., Rao, S. L. N. and Sarma, P. S. (1963) Some structural features and neurotoxic action of a compound from Lathyrus sativus seeds. Current Science 4:153-155.

Ahlskog, J.E., Waring, S.C., Kurland, L.T., Petersen, R.C., Moyer, T.P., Harmsen, W.S., araganore, D.M., O'Brien, P.C., Esteban-Santillan, C. and Bush, V. (1995) Guamanian neurodegenerative disease: Investigation of the calcium metabolism/heavy metal hypothesis. Neurol., 45:13401344.

Ahlskog, J.E., Petersen, R.C., Waring, S.C., Esteban-Santillan, C., Craig, U.K., Maraganore, D.M., Lennon, V.A. and Kurland, (1997) Guamanian neurodegenerative disease: Are diabetes mellitus and altered humoral immunity clues to pathogenesis? Neurology 48:1356-1362.

Albretsen J.C., Khan S.A., Richardson J.A. (1998) Cycad palm toxicosis in dogs: 60 cases (1987-1997) J. Am. Vet. Med. Assoc., 213: 99-101.

Allen C.N., Omelchenko I., Ross S.M. and Spencer P. (1995) The neurotoxin, $\beta$ - $N$-methylamino-L-alanine (BMAA) interacts with the strychnine-insensitive glycine modulatory site of the $N$-methyl-D-aspartate receptor. Neuropharmacol., 34 : 651-658.

Andersson H., Lindqvist E. and Olson L. (1997) Plant-derived amino acids increase hippocampal BDNF, NGF, $c$-fos and $h s p 70$ mRNAs. NeuroReport., 8:1813-1817.

Arnold, A., Edgren, D. C. and Palladino, V. S. (1954) Amyotrophic lateral sclerosis. J. Nerv. Ment. Dis., 117:135-139.

Banack, S. A. and Cox, P. A. (2003a) Biomagnification of cycad neurotoxins in flying foxes. Implications for ALS-PDC in Guam. Neurology 61:387-389.

Banack S.A. and Cox P.A. (2003b) Distribution of the neurotoxic nonprotein amino acid BMAA in Cycas mi- cronesia. Botanical J. Linnean Soc., 143: 165-168.

Banack, S. A., , S. J. and Cox, P. A. (2006) Neurotoxic flying foxes as dietary items for the chamorro people, Marianas Islands. J. Ethnopharmacol., 106:97-104.

Brenner, E.D., Martinez-Barboza, N., Clark, A.P., Liang, Q.S., Stevenson, D.W: and Coruzzi, G.M. (2000) Arabidopsis mutants resistant to $\mathrm{S}(+)-$ $\beta$-methyl- $\alpha, \beta$-diaminopropionic acid, a cycad-derived glutamate receptor agonist. Plant Physiol., 124:16151624.

Brenner E.D., Stevenson D.W., McCombie R.W., Katari M.S., Rudd S.A., Mayer K.FX., Palenchar P.M., Runko S.J., Twigg R.W., Dai G., Martienssen R.A., Benfey P.N. and Coruzzi G.M. (2003a) Expressed sequence tag analysis in Cycas, the most primitive living seed plant. Genome Biol., 4:R78:1-11.

Brenner E.D., Stevenson D.W. and Twigg R.W. (2003b) Cycads: evolutionary innovations and the role of plant-derived neurotoxins. Trends in Plant Science 8:446-452.

Brownson D.M., Mabry T.J. and Leslie S.W. (2002) The cycad neurotoxic amino acid, $\beta-N$-methylamino-Lalanine (BMAA), elevates intracellular calcium levels in dissociated rat brain cells. J. Ethnopharmacol., 82:159-167.

Campbell, M.E., Mickelsen, O., Yang, M.G. and Laqueur, G.L., Keresztesty JC (1966) Effects of strain, age and diet on the response of rats to the ingestion of Cycas circinalis. J. Nutr., 88:115-124.

Caparros-Lefebvre, D., Elbaz, A. and the Caribbean Parkinsonism Study Group (1999) Possible relation of atypical parkinsonism in the French West Indies with consumption of tropical plants: a case-control study. Lancet 354(ii):281-286.

Chang Y.-C., Chiu S.-J. and Kao K.-P. (1993) Beta-N-meethylamino-L- 
alanine (L-BMAA) decreases brain glutamate recptor number and induces behavioral changes in rats. Chin. J. Physiol., 36:79-84.

Charlton, T.S., Marini, A.M., Markey, S.P., Norstog, K. and Duncan, M.W. (1992) Qunatification of the neurotoxin2-amino-3-(methylamino) propanoic acid (BMAA) in Cycadales. Phytochem., 31:3429-3432.

Chen, X., Xia, Y., Gresham, L.S., Molgaard, C.A., Thomas, R.G., Galasko, D., Wiederholt, W.C. and Saitoh, T. (1996) ApoE and SYP2D6 polymorphism with and without parkinsonism-dementia complex in the people of Chamorro, Guam. Neurology 47:779-784.

Chiu, J, DeSalle, R, Lam, HM, Meisel, L, Coruzzi, G (1999) Molecular evolution of glutamate receptors: a primitive signaling mechanism that existed before plants and animals diverged. Mol Biol Evol. Jun;16(6):826-38

Copani A., Canonico P.L. and Nicoletti F. (1990) $\beta$ - $N$-methylamino-L-alanine (L-BMAA) is a potent agonist of 'metabolotropic' glutamate receptors. Eur J. Pharmacol., 181: 327-328.

Copani A., Canonico P.L., Catania M.V., Aronica E., Bruno V., Ratti E., van Amsterdam F.T.M., Gaviraghi G. and Nicoletti F. (1991) Interaction between $\beta$ - $N$-methylamino-L-alanine and excitatory amino acid receptors in brain slices and neuronal cultures. Brain Res., 558: 79-86.

Cohen, S.A. and Michaud, D.P (1993) Synthesis of a fluorescent derivatizing reagent, 6-aminoquinolyl-Nhydroxysuccinimidyl carbamate, and its application for the analysis of hydrolysate amino acids via high performance liquid chromatography. Anal. Biochem., 211:279-287.

Cox, P. A. and Sacks, O. W. (2002) Cycad neurotoxins consumption of flying foxes, and ALS-PDC diseases in Guam. Neurology 58:956-959.

Cox, P.A., Banack, S. A. and Murch, S. J. (2003) Biomagnification of cyanobacterial neurotoxins and neurodegenerative diseases among the Chamorro people of Guam. Proc. Natl. Acad. Sci., 100:13380-13383.

Cox, P. A., Banack, S. A., Murch, S. J., Rasmussen, U. Tien, G., Bidigare, R. R., Metcalf, J. S., Morrison, L. F.,
Codd, G. A. and Bergman, B. (2005) Diverse taxa of cyanobactreria produce $\beta$-N-methylamino-L-alanine, a neurotoxic amino acid. Proc. Natl. Acad. Sci., 102:5074-5078.

Cruz-Aguado, R., Winkler, D. and Shaw, C.A. (2006) Lack of behavioural and neuropathologial effects of dietary $\beta$-methylamino-L-alanin (BMAA) in mice. Pharmacol. Biochem. Behaviour Jun;84(2):294-9

Dastur, D.K. (1964) Cykad toxicity in monkeys: clinical, pathological and biochemical aspects. Fed. Proc., 23:1368-1369.

Dastur D.K. and Palekar R.S. (1974) The experimental pathology of cycad tocixity with special reference to oncogenic effects. Ind. J. Cancer 11:33-49.

Dawson, R., Marschall, E.-G., Chan, K.C., Millard, W.J., Eppler, B. and Patterson, T.A. (1998) Neruochemical and neurobehavioral effects of neonatal administration of $\beta-N$ methylamino-L-alanine and 3, 3'iminodipropionitrile. Neurotoxicol Teratol. 20:181-192.

Dobat K., Peikert-Holle T. (1985) Blüten und Fledermäuse. Waldemar Kramer, Frankfurt am Main

Dossaji, S.F. and Bell, E.A. (1973) Distribution of $\alpha$-amino- $\beta$ methylaminopropionic acid in Cycas. Phytochem., 12:143-144.

Duncan, M.W. (1991) Role of the cycad neurotoxin BMAA in the amyotrophic lateral-sclerosis-parkinsonism dementia complex of the western pacific. Adv. Neurol., 56:301-310.

Duncan, M. W., Kopin, I. J., Garruto, R. M., Lavine, L. and Markey, S. P. (1988) 2-amino-3 (methylamino)propionic acide in cycad-derived foods is an unlikely cause of amyotrophic lateral sclerosis/parkinsonism. The Lancet ii:631-632.

Duncan. M. W., Kopin, I. J., Crowley, J. S., Jones, S. M. and Markey, S. P. (1989) Quantification of the putative neurotoxin 2-amino-3(methylamino)propanoic acid (BMAA) in cycadales: Analysis of the seeds of some members of the faily cycadaceae. J. Anal. Toxicol., 13: 169-175.

Duncan, M. W., Steele, J. C., Kopin, I. J. and Markey, S. P. (1990) 2-Amino- 
3-(methylamino)-propanoic acid (BMAA) in cycad flour: An unlikely cause of amyotrophic lateral sclerosis and parkinsonism-dementia of Guam. Neurology 40:767-772.

Duncan MW, Villacreses NE, Pearson PG, Wyatt L, Rapoport SI, Kopin IJ, Markey SP, Smith QR (1991) 2Amino-3-(methylamino-propanoic acid (BMAA) Pharmacokinetics and blood-brain barrier permeability in the rat. J. Pharm. Exp. Ther., 258:27-35.

Duncan, M. W., Marini, A. M., Watters, R., Kopin, O. J. and Markey, S. P. (1992) Zinc, a neurotoxin to cultured neurons, contaminates cycad flour prepared by traditional guamanian methods. J. Neurosci., 12:1523-1537.

Durlach, J., Bac, P., Durlach, V., Durlach, A., Vara, M. amnd Guiet-Bara, A. (1997) Are age-related neurodegenerative diseases linked with various types of magnesium depletion? Magnesium Res., 10:339-353.

Einarsson, S., Josefsson, B. and Lagerkvist, S. (1983) Determination of amino acids with 9fluorenylmethyl chloroformate and reveresed phase high performance liquid chromatography. J. Chromatogr., 282:609-618.

Esclair F., Kisby G., Spencer P., Milne J., Lesort M., Hugon J. (1999) The Guam cycad toxin methylazoxymethanol damages neuronal DNA and modulates tau mRNA expression and excitotoxicity. Exp Neurol.

155:11-21.

Forsythe, ID, Barnes-Davies, M (1997) Synaptic transmission: well-placed modulators. Curr Biol. Jun 1;7(6):R362-5. Review

Fosberg F.R. (1964) Resume of the Cycadaceae. Fed. Proc., 23:1340-1342.

Gajdusek D.C. and Salazar A.M. (1982) Amyotrophic lateral sclerosis and parkinsonian syndromes in high incidence among the Ayuy and Jakai people of West New Guinea. Neurology 32:107-126

Gajdusek, D.C. (1985) Hypothesis: interference with axonal transport of neurofilament as a common pathogenetic mechanism in certain diseases of the central nervous system. N. Engl. J. Med., 312:714-719.
Gannon, R.L. and Terrian D.M. (1989) BOAA selectively enhances Lglutamate release from guinea pig hippocampal mossy fiber synaptosomes. Neurosci. Lett., 107:289-294.

Garruto, R.M. and Yase, Y. (1974) Neurodegenerative disorders of the Pacific. Trends Neurosci., 9:368-374.

Garruto R.M, Fukatsu R., Yangihara R, Gajdusek D.C, Hook G, Fiori C.E. (1984) Imaging of calcium and aluminum in neurofibrillary tanglebearing neurons in parkinsonismdementia of Guam. Proc. Natl. Acad. Sci. U S A., 81:1875-1879.

Garruto, RM, Yanagihara, R, Gajdusek, DC (1985) Disappearance of highincidence amyotrophic lateral sclerosis and parkinsonism-dementia on Guam.Neurology. Feb;35(2):193-8

Garruto R.M., Swyt C., Yangihara R., Fiori C.E., Gajdusek D.C. (1986) Intraneuronal co-localization of silicon with calcium and aluminum in amyotrophic lateral sclerosis and parkinsonism with dementia of Guam. N. Engl. J. Med., 315:711-712.

Garruto, R.M., Yanagihara, R., Shankar, S.K. et al (1988a) Experimental models of metal-induced neurofibrillary degeneration. In: Tsubaki $\mathrm{T}$ and Yase Y (Eds.) Amyotrophic lateral sclerosis, Elsevier, Amserdam, pp. 41-50.

Garruto, R.M., Yanagihara, R. and Gajdusek DC (1988b) Cycads and amyotrophic lateral sclerosis/parkisonism dementia. Lancet ii:1079.

Gibbs, C.J.Jr. and Gajdusek, D.C. (1982) An update on longterm in vivo and in vitro studies designed to identify a virus as the cause of amyotrophic lateral sclerosis, parkinsonismdementia, and Parkinson's disease. In: Human Motor Neuron Diseases, L.P. Rowland (Ed.), Raven Press, New York, pp. 343-353.

Gobe, G.C. (1994) Apoptosis in brain and gut tissue of mice fed a seed preparation of the cycad Lepidozamia peroffskyana. Biochem. Biophys. Res. Commun., 205:327-333.

Haddock, RL, Santos, JV (1992) Are the endemic motor neuron diseases of Guam really disappearing? Southeast Asian J Trop Med Public Health. Jun;23(2):278-81 
Hashmi, M. and Anders, M.W. (1991) Enzymatic reaction of $\beta-\mathrm{N}-$ methylaminoalanine with L-amino acid oxidase. Biochim. Biophys. Acta 1074:36-39.

Hermosura M.C, Nayakanti H., Dorovkov M.V., Calderon F.R., Ryazanov A.G., Haymer D.S, Garruto R.M. (2005) A TRPM7 variant shows altered sensitivity to magnesium that may contribute to the pathogenesis of two Guamanian neurodegenerative disorders. Proc. Natl. Acad. Sci. USA, 102:11510-11515.

Hooper, P. T. (1978) Cycad poisoning in Australia - etiology and pathology. In: Effects of Poisonous Plants on Livestock, R. F. Keeler, R. F. Van Kampen and L. F. James (Eds.), Academic Press, New York, pp. 337-347.

Hooper, P.T. (1983) Cycad poisoning. In: Handbook of Natural Toxins, Plants and Fungal Toxins. Vol. 1, R.F. Keeler and A.T. Tu (Eds.), New York, Marcel Dekker, pp. 463-469.

Ibáňez y Garcia L de. (1871) The course of history in the Mariana, Caroline and Palau Islands from the time of their discovery by Magellan in 1521 to the present. Translated and annotated by Marjorie Driver. Micronesian Area Research Centre (MARC), University of Guam, 1987, pp. 2-3.

ICARDA (1995) International Center for Agricultural Research in the Dry Areas, 1995: Germplasm Program (Legumes) Annual Report for 1995, pp. 167-169.

Ikonomidou, C, Turski, L (1996) Prevention of trauma-induced neurodegeneration in infant and adult rat brain: glutamate antagonists. Metab Brain Dis. Jun;11(2): 125-41

Ikonomidou C, Qin Qin Y, Labruyere J, Olney JW (1996) Motor neuron degeneration induced by excitotoxin agonists has features in common with those seen in the SOD-1 transgenic mouse model of amyotrophic lateral sclerosis. J Neuropathol Exp Neurol. Feb;55(2):211-24.

Khabazian, I., Bains, J.S., Williams, D.E., Cheng, J., Wilson, J.M.B., Pasqualotto, B.A., Pelech, S.L., Andersen, R.J., Wang, Y.-T., Liu, I., Nagai, A., Kim, S.U., Craig, U.-K. and Shaw, C.A. (2002) Isolation of various forms of sterol $\beta$-D-Glucoside from the seed of Cycas circinalis: neurotoxicity and implications for ALS-parkinsonism dementia complex. J. Neurochem., 82:516-528.

Kisby G.E., Dwijendra N.R, Spencer P.S. (1988) Determination of $\beta-N$ methylamino-L-alanine (BMAA) in plant (Cycascircinalis L.) and animal tissue by precolumn derivatizatioin with 9-fluorenylmethyl chloroformate (FMOC) and reversed-phase highperformance liquid chromatography. J Neurosci. Meth. 26:45-54.

Kisby, G. E, Ellison, M. and Spencer, P. S. (1992) Content of the neurotoxins cycasin (methylazoxmethanol $\beta$ D-glucoside) and BMAA ( $\beta-\mathrm{N}$ methylamino-L-alanine) in cycad flour prepared by Guam Chamorros. Neurology 42:1336-1340.

Klein M., Martinoia E., HoffmanThoma G. and Weissenböck G. (2000) A membrane-potential dependent $\mathrm{ABC}$-like transporter mediates the vacuolar uptake of rye flavone glucuronides: regulation of glucuronide uptake by glutathione and ist conjugates. The Plant Journal 21(3): 289-304.

Koerner D.R. (1952) Amyotrophic lateral sclerosis on Guam. Ann. Internat. Med., 37:1204-1220.

Kurland L.T. (1972) An appraisal of the neurotoxicity of cycad and the etiology of amyotrophic lateral sclerosis on Guam. Fed. Proc., 31:1540-1542.

Kurland, L.T. (1988) Amyotrophic lateral sclerosis and Parkinson's disease complex on Guam linked to an environmental toxin. Trends Neurosci., 11:51-53.

Kurland L.T. and Mulder D.W. (1954) Epidemiologic investigations of amyotrophic lateral sclerosis. I. Preliminary report on geographic distribution and special reference to the Mariana Islands, including clinical and pathologic observations. Neurology 4:355-378 and 438-448.

Kurland LT, Radhakrishnan K, Williams DB, Waring SC (1994) Amyotrophic lateral sclerosis-parkinsonismdementia complex on Guam: epidemiologic and etiological perspectives, in Motor neuron Disease (Williams 
A, Ed.) Chapman\&Hall, London, pp. 109-130.

Lam, HM, Hsieh, MH, Coruzzi, G (1998a) Reciprocal regulation of distinct asparagine synthetase genes by light and metabolites in Arabidopsis thaliana. Plant J. Nov;16(3):345-53

Lam, HM, Chiu, J, Hsieh, MH, Meisel, L, Oliveira, IC, Shin, M, Coruzzi, G (1998b) Glutamate-receptor genes in plants. Nature. Nov 12;396(6707):125-6

Lam, HM, Peng, SS, Coruzzi, GM (1994) Metabolic regulation of the gene encoding glutamine-dependent asparagine synthetase in Arabidopsis thaliana. Plant Physiol.

Dec;106(4):1347-57

Lemke, T.O. (1992) History of fruit bat use, research, and protection in the Northern Mariana Islands. In: Pacific Island Flying Foxes: Proceedings of an International Conference, D.E. Wilson and G.L. Graham (Eds.), Biological Report 90(23), U.S. Fish and Wildlife Service, Washington D.S., pp. 135-142.

Lewis, M.D., McQueen, I.N.F. and Scanlon M.F. (1990) Motor neurone disease serum and $\beta-N$-methylaminoL-alanine stumulate thyrotrophinreleasing hormone production by cultured brain cells. Brain Res., 537:251-255.

Li, C., Brownson, D.M., Mabry, T.J., Perera, C. and Bell, E.A. (1996) Nonprotein amino acids from seeds of Cycas circinalis and Phaseolus vulgaris. Phytochem., 42:443-445.

Lindström, H., Luthman, J., Mouton, P., Spencer, P. and Olson, L. (1990) Plant-derived neurotoxic amino acids ( $\beta$ - $N$-oxalylamino-L-alanine and $\beta-N$ methylamino-L-alanine): effects on central monoamine neurons. J.

Neurochem., 55:941-949.

Lujan, R. J. (1992) Local policies and protection by the government of Guam. In Pacific island flying foxes. Proceedings of an International Conference, D. E. Wilson and G. L. Graham (Eds.), Biological report 90( 23 ). U.S. Fish and Wildlife Service, Washington, D.C., pp. 131-134.

Manzoni, O.J.J., Prezeau, L. and Bockaert, J. (1991) $\beta-N$ methylamino-L-alanine is a low- affinity agonist of metabotropic glutamate receptors. NeuroReport. 2:609-611.

Martin, R.B. (1979) Amino acids and derivatives as ambidentate ligands. In: Sigel, H. (Ed.) Metal ions in biological systems, Dekker, New York, vol. 9, pp. 1-39.

Mason, M. M. and M. G. Whiting. 1966. Demylelination in the bovine spinal cord caused by Zamia neurotoxicity. Fed. Proc., 25:533.

Matsuoka, Y., Rakonczay, Z., Giacobini, E. and Naritoku, D. (1993) L- $\beta$ Methylamino-alanine-incuced behavioral changes in rats. Pharmacol Biochem. Behav., 44:727-734.

McNamara, D, Dingledine, R (1990) Dual effect of glycine on NMDAinduced neurotoxicity in rat cortical cultures. J Neurosci.Dec; 10(12):3970-6

Medina, J.H, Izquierdo, I (1995) Retrograde messengers, long-term potentiation and memory. Brain Res Brain Res Rev. Sep;21(2):185-94. Review

Miller, G. (2006) Guam's deadly stalker: On the loose worldwide? Science 313:428-431.

Moore, S., Spackman, D.H. and Stein, W.H. (1958) Automatic recording apparatus for use in the chromatography of amino acids. Fed. Proc., 4:1107-1115

Monson, C.S., Banack, S.A: and Cox, P.A. (2003) Conservation implications of Chamorro consumption of flying foxes as a possible cause of amyotrophic lateral sclerosis - parkinsonism dementia complex in Guam. Conservation Biology 17:678-686.

Montine, T. J., Li, K., Perl, D. P. And Glasko, D. (2005) Lack of $\beta$ methylamino-L-alanine in brain from controls, $\mathrm{AD}$, or Chamorros with PDC. Neurology 65:768-769.

Muller, D, Joly, M, Lynch, G (1988) Contributions of quisqualate and NMDA receptors to the induction and expression of LTP. Science. Dec 23;242(4886):1694-7 
Murch, S. J., Cox, P. A. and Banack, S. A. (2004a) A mechanism for slow release of biogmagnified cyanobacterial neurotoxins and neurodegenerative disease in Guam. Proc. Natl. Acad. Sci. USA, 101:12228-12231.

Murch, S. J., Cox, P. A., Banacj, S. A, Steele, J. C. And Sacks, O. W. (2004b) Occurrence of $\beta$ methylamino-L- alanine (BMAA) in ALS/PDC patients from Guam. Acta Neurol. Scand., 110:267-269.

Murti, V.V.S., Seshadri, T. R. and Venkitasubarmanian, T. A. (1964) Neurotoxic compounds of the seeds of Lathyrus sativus. Phytochem., 3:73-78.

Myers, T. G. and Nelson, S. D. (1990) Neuroactive carbamate adducts of $\beta$ methylamino-L- alanine and Ethylenediamine. Detection and quantitation under physiological conditions by ${ }^{13} \mathrm{C}$ NMR. J. Biol. Chem., 265:10193-10195.

Nedeljkov, V., Lopicic, S., Pavlovic, D. and Cemerikic, D. (2005) Electrophysiological effect of $\beta-N$ methylamino-L-alanine on retzius nerve cells of the leexh Haemopis sanguisuga. Ann N.Y. Acad. Sci., 1048:349-351.

Nishida, K., Kobayashi, A. and Nagahama, T. (1955) Studies on cycasin, a new toxin glycoside pf Cycas revoluta Thunb. Part 1. Isolation and the structure of cycasin. Bull. Agr. Chem. Soc. Jap., 19:77-84.

Nunn, P.B. and O'Brien, P. (1989) The interaction of $\beta$-N-methylamino-Lalanine with bicarbonate: an $1 \mathrm{H}-$ NMR study. FEBS Letters 251:31-35.

Nunn, P.B., Seelig, M., Zagoren, J.C. and Spencer, P.S. (1987) Stereospecific acute neuronotoxicity of 'uncommon' plant amino acids linked to human motor-system diseases. Brain Res., 410:375-379.

Nunn, P.B., O'Brian, P., Pettit, L.D. and Pyburn, S.I. (1989) Complexes of Zinc, Copper, and Nickel with the nonprotein amino acid L- $\alpha$-amino- $\beta$ methylaminopropionic acid: A naturally occuring neurotoxin. J. Inorg. Biochem., 37:175-183.

Oh, C., Brownson, D.M. and Mabry, T.J. (1995) Screening for non-protein amino acids in seeds of the Guam cycad, Cycas circinalis, by an improved GC-MS method. Planta Medica 61:66-70.

Oliveira, IC, Coruzzi, GM (1999) Carbon and amino acids reciprocally modulate the expression of glutamine synthetase in Arabidopsis. Plant Physiol. Sep;121(1):301-10.

Pai, K.S., Shankar, S.K. and Ravindranath V. (1993a) Billionfold difference in the toxic potencies of two excitatory plant amino acids, L-BOAA and L-BMAA: bichemical and morphological studies using mouse brain slices. Neurosci. Res., 17:241-248.

Pai, K.S. and Ravindranath, V. (1993b) L-BOAA induces selective inhibition of brain mitochondrial enzyme, NADH-dehydrogenase. Brain Res., 621:215-221.

Pan, M., Mabry, T.J., Cao, P. and Moini, M. (1997) Identification of nonprotein amino acids from cycad seeds as N-ethoxycarbonyl ethyl ester derivatives by positive chemicalionization gas chromatography-mass spectrometry. J. Chromatogr., 787:288-294.

Perry, T.L., Bergeron, C., Biro, A.J. and Hansen S. (1989) $\beta$ - $N$-MethylaminoL-alanine Chronic oral administration is not neurotoxic to mice. J. Neurol. Sci., 94:173-180.

Plato, C.C., Galasko, D., Garruto, R.M., Plato, M., Gamst, A, Craig, U.-K., Torres, J.M. and Wiederholt W. (2002) ALS and PDC of Guam. Forty-year follow-up. Neurology 58:765-773.

Poorkaj, P., Tsuang, D., Wijsman, E., Steinbart, E., Garruto, R.M., Craig, U.-K., Chapman, N.H., Anderson, L., Bird, T.D., Plato, C.C., Perl, D.P., Weiderholt, W., Galasko, D. and Schellenberg, G.D. (2001) TAU as a susceptibility gene for amyotropic lateral sclerosis-parkinsonism dementia complex of Guam. Arch. Neurol., 58:1871-1878.

Polsky, F.I., Nunn, P.B. and Bell, E.A. (1972) Distribution and toxicity of $\alpha$ amino- $\beta$-methylaminopropionic acid. Fed. Proc., 31:1473-1475.

Rakonczay, Z., Matsuoka, Y. and Giacobini, E. (1991) Effects of L- $\beta-N$ methylamino-L-alanine (L-BMAA) on the cortical cholinergic and gluta- 
matergic systems of the rat. J. Neurosci. Res., 29:121-126.

Rao, S.D., Banack, S.A., Cox, P.A. and Weiss, J.H. (2006) BMAA selectively injures motor neurons via AMPA/kainate receptor activation. Exp. Neurol., Sep 201(1):244-252

Reed, DM, Brody, JA (1975) Amyotropihic lsteral sclerosis and parkinosinism-dementia on Guam, 19451972. I. Descriptive epidemiology. Am J Epidemiol; 101:287-301.

Reed, D., Labarthe, D., Chen, K.M. and Stallones, R. (1987) A cohort study of amyotrophic lateral sclerosis and parkinsonism-dementia on Guam and Rota. Am. J. Epid., 125:92-100.

Ressler, C., Redstone, P.A. and Erenberg, R.H. (1961) Isolation and identification of a neuroactive factor from Lathyrus latifolius. Science, 134:188-190.

Richter, K.E. and Mena, E.E. (1989) L$\beta$-Methylaminoalanine inhibits $\left[{ }^{3} \mathrm{H}\right]$ glutamate binding in the presence of bicarbonate ions. Brain Res., 492:385-388.

Riggs, N.V. (1956) Glucosyloxyazoxymethane, a constituent of the seeds of Cycads circinalis. Chem. Ind., 926.

Román, G.C. (1996) Neuroepidemiology of amyotrophic lateral sclerosis: clues to aetiology and pathogenesis. J. Neurol. Neurosurg. Psychiatry 61:131-137.

Ross, S.M., Seelig, M. and Spencer, P.S., (1987) Specific antagonism of excitotocix action of 'uncommon' amino acids assayed in organotypic mouse cortical cultures. Brain Res., 425:120-127.

Rowland L.P. (1991) Ten central themes in a decade of ALS research. Adv. Neurol., 56:3-23.

Sacks O.W. (1993) Guam ALS-PDC: possible causes. Science 262:826.

Sayer, T.L. and Rabenstein, D.L. (1976) Can. J. Chem., 54:3392-3397.

Schaefer, W.H. (2006) Reaction of primary and secondary amines to form carbamic acid glucuronides. Curr. Drug Metab., 7:873-881.

Schneider, D., Wink, M. and Sprorer, F. (2002) cycads: their evolurion, toxins, herbivores and insect pollinators. Natruwissenschaften 89:281-294.

Seawright, A.A., Brown, A.W., Nolan, C.C. and Cavanagh, J.B. (1990) Se- lective degeneration of cerebellar cortical neurons caused by cycad neurotoxin, L- $\beta$-methylaminoalanine (LBMAA), in rats. Neuropathol. Appl. Neurobiol., 16:153-169.

Silbernagl, S. (1988) The renal handling of amino acids and oligopeptides. Phys. Rev., 68: 911-1007.

Smith, S.E. and Meldrum, B.S. (1990) Receptor site specificity for the acute effects of $\beta-N$-methylamino-L-alanine in mice. Eur. J. Pharmacol., 187:131-134.

Smith, Q.R., Nagura, H., Takad, a Y. and Duncan, M.W. (1992) Facilitated transport of the neurotoxin, $\beta-N$ methylamino-L-alanine, across the blood-brain barrier. J. Neurochem., 58:1330-1337.

Spencer, P.S. (1989) Western Pacific ALS-parkinsonism-dementia: a model of neuronal aging triggered by environmental toxins. In: Parinsonism and Aging, D.B. Calne (Ed.), New York, Raven Press, p. 133-144.

Spencer, P.S., Nunn, P.B., Hugon, J., Ludolph, A.C., Ross, S.M., Roy, D.N. and Robertson, R.C. (1987a) Guam amyotrophic lateral sclerosisParkinsonism-Dementia linked to a plant excitant neurotoxin. Science 237:517-522.

Spencer, P.S., Palmer, V.S., Herman, A. and Asmedi, A. (1987b) Cycad use and motor neurone disease in Irian Jaya. Lancet ii:1273-1274.

Spencer, P.S., Palmer, V.S., Herman, A. and Asmedi, A. (1987c) Cycad use and motor neurone disease in Kii peninsula of Japan. Lancet ii:1462-1463.

Spencer P.S., Hugon J., Ludolph A.C., Nunn P.B., Ross S.M., Roy d.N., Schaumburg H.H. (1987d) Discovery and partial characterization of primate motor-system toxins. CIBA Found. Symp., 126:221-237.

Spencer P.S., Kisby G.E., Ludolph A.C. (1991a) Long-latency neurodegenerative disease in the western Pacific. Geriatrics 46(Suppl 1):37-42.

Spencer P.S., Kisby G.E, Ludolph A.C. (1991b) Slow toxins, biologic markers, and long-latency neurodegenerative disease in the western Pacific region. Neurology 41(Suppl. 2):62-68. Steele, J.C. and Guzman, T. (1987) Observations about amyotrophic lateral sclerosis and the parkinsonism- 
dementia complex of Guam with regard to epidemiology and etiology. Can. J. Neurol. Sci., 14:358-362.

Tate, G. H. H. (1934) Bats from the Pacific Islands including a new fruit bat from Guam. American Museum Novitates 713: 1-3.

Thomsen, C., Mulvihill, E.R., Haldeman, B., Pickering, D.S., Hampson, D.R. and Suzdak, P.D. (1993) A pharmacological characterization of the mGluR $1 \alpha$ subtype of the metabotropic glutamate receptor expressed in a cloned baby hamster kidney cell line. Brain Res., 619:22-28.

Tsien, JZ, Huerta, PT, Tonegawa, S (1996) The essential role of hippocampal CA1 NMDA receptordependent synaptic plasticity in spatial memory. Cell. Dec 27;87(7):1327-38

Vega, A. and Bell, E. A. (1967) $\alpha$ amino- $\beta$-methylaminopropionic acid, A new amino acid from seeds of $C y$ cas circinalis. Phytochem., 6:759-762.

Vega, A., Bell, E. A. and Nunn, P. B. (1968) The preparation of L- and D$\alpha$-amino- $\beta$ - methylaminopropionic acid and the identification of the compound isolated from Cycas circinalis as the L-isomer. Phytochem., 7:1885-1887.

Wang, T.-C.L., Shih, M.-c., Markey, S.P. and Duncan, M.W. (1989) Quantitative analysis of low molecular weight polar compounds by continuous flow liquid secondary ion tandem mass spectrometry. Anal. Chem., 61:1013-1016.

Waring, S.C., Esteban-Santillan, C., Ree, d D.M., Craig, U-K., Labarthe, D.R., Petersen, R.C. and Kurland, L.T. (2004) Incidence of amyotrophic lateral sclerosis and of the parkinsonism-dementia complex of Guam, 1950-1989. Neuroepidemiol., 23:192-200.

Weiss, J.H. and Choi, D.W. (1988) Beta-N-methylamino-L-alanine neurotoxicity: requirement for bicarbonate as a cofactor. Science 241: 973-975.

Weiss, J.H., Christine, C.W. and Choi D.W. (1989a) Bicarbonate dependence of glutamate receptor activation by $\beta-N$-methylamino-L-alanine: channel recording and study with re- lated compounds. Neuron., 3:321-326.

Weiss, J.H., Koh, J.-Y. and Choi, D.W. (1989b) Neurotoxicity of $\beta-N$ methylamino-L-alanine (BMAA) and $\beta$ - $N$-oxalylamino-L-alanine (BOAA) on cultured cortical neurons. Brain Res., 497: 64-71.

Whiting M.G. (1963) Toxicity of the cycads. Econ. Bot., 17:271-302.

Whiting, M.G. (1988) Toxicity of cycads: implications for neurodegenerative diseases and cancer: transcripts of four cycad conferences (19621967). New York: Third World Research Foundation, in collaboration with the Lyon Arboretum, University of Hawaii.

Wiles, G. J. (1987) The status of fruit bats on Guam. Pacific Science 41:148-157.

Wiles G.J. and Payne N.H. (1986) The trade in fruit bats Pteropus spp. on Guam and other Pacific islands. Biological Conservation 38:143-161. Wiles G.J., Aquon C.F., Davis G.W. (1995) The status and distribution of endangered animals and plants in Northern Guam. Micronesica 28:31-49.

Wilson, J. and Shaw, C.A. (2006) Commentary on: Return of the cycad hypothesis - does the amyotrophic lateral sclerosis/parkinsonism dementia complex (ALS/PDC) of Guam have bew implications for global health? Neuropathol. Appl. Neurobiol., 32:341-343.

Wilson, J. M. B., Khabazian, I., wong, M. C., Seyedalikhani, A., Bains, J. S., Pasqualotto, B. A., Williams, D. E., Andersen, R. J., Simpson, R. J., Smith, r., Craig, U.-K-, Kurland, L. T. and Shaw, C. A. (2002) Behavioral and neurological correlated of ALSparkinsonism dementia comlpex in adult mice fed washed cycac flour. NeuroMolecular Med., 1:207-221.

Wilson J.M.B., Khabazian I., Pow D.V., Craig U.K., Shaw C.A.(2003) Decrease in Glial Glutamate Transporter Variants and Excitatory Amino Acid Receptor Down-Regulation in a Murine Model of ALS-PDC. NeuroMolecular Med., 3:105-118.

Wilson J.M., Petrik M.S., Grant S.C., Blackband S.J., Lai J., Shaw C.A. (2004) Quantitative measurement of 
neurodegeneration in an ALS-PDC model using MR microscopy. NeuroImage 23: 336-342.

Wilson J. and Shaw C.A. (2006) Commentary on: Return of the cycad hypthesis - does the amyotrophic lateral sclerosis/parkinsonism dementia complex (ALS/PDC) of Guam have new implications for global health? Neuropat. Appl. Neurobiol., 32; 341-343.

Worms, P.M. (2001) The epidemiology of motor neuron diseases: a review of recent studies. J. Neurol. Sci., 191:3-9.

Yang, M.G., Mickelsen, O., Campbell, M.E., Lqueur, G.L. and Keresztesy, J.C. (1966) Cycad flour used by guamanians: effects produced in rats by long-term feeding. J. Nutr., 90:153-156.

Yase, Y. (1972) The pathogenesis of amyotrophic leteral sclerosis. Lancet ii:292-296.

Yase Y. (1979) ALS in the Kii Peninsula: one possible etiological hypothesis. Pages 307-318 in T. Tsubaki and Y. Toyokura, editors. Amyotrophic lateral sclerosis. University Tokyo Press

Yase, Y. (1980) The role of aluminum in CNS degeneration with interaction of calcium. Neurotoxicol., 1:101-109.
Yase Y. (1987) The pathogenetic role of metals in motor neuron disease the participation of aluminum. Adv Exp Med Biol. 1987;209:89-96.

Yasuda, N. and Shimizu, T. (1998) Cycad poisoning in cattle in Japan Studies on spontaneous and experimental cases. J. Toxicol. Sci., 23 (Suppl. II):126-128.

Zeevalk, G.D. and Nicklas, W.J. (1989) Acute excitotoxicity in chick retina caused by the unusual amino aicds BOAA and BMAA: effects of MK801 and kynurenate. Neurosci. Letters 102:284-290.

Zeevalk, G.D. and Nicklas, W.J. (1994) Nitric oxide in retina: relation to excitatory amino acids and excitotoxicity. Exp. Eye Res., 53:343-350.

Zhang Z.X., Anderson D.W., Mantel N., Román G.C. (1996) Motor neuron disease on Guam: geographic and familial occurrence 1956-85. Acta Neurol Scand., 94:51-59.

Zimmerman H (1945) Progress report of work in the laboratory of pathology during May, 1945, Guam. US Naval Medical Research Unit Number 2, June 1, Washington DC, 1945. 



\section{Annex 1}

\section{Summaries of epidemiological studies}

Steele and Guzman (1987) observed that the ALS/PD complex only occurred where cycad trees grow and where people use its seeds for food and medicine. They provided an explanation for the rarity of the disease on the island of Saipan. During Spanish and German administrations, before 1914, cycad trees were abundant and people used cycad flour in the same way as the people of Guam and Rota did. When the Japanese occupied Saipan in 1914 they cleared large parts of the island for sugar plantation and by the 1920s most of the cycad forests had been cut down. When neurological surveillance teams visited Saipan in the 1950s cycad flour had not been used on the island for 30 years. At that time ALS was less common there than on Guam or Rota, and Saipanese people that suffered the disease were born before 1917. Another fact that is related is that the disease was not seen in Filipinos who arrived on Guam after 1950, when the flour ceased to be a staple food. Interwievs that Steele and Guzman conducted suggested that familial aggregation of patients could relate to differences between families in the method of preparing the cycad seeds. There appeared to be considerable differences in regard to the soaking process and the number of water changes between families (Steele and Guzman, 1987).

The US National Institute of Neurological Disorders established a registry for cases of motor neuron disease and parkinson dementia complex on Guam in 1956. This case registry was used by Román and coworkers (1996) to analys the epidemiological temporal pattern of occurrence of motor neuron disease on Guam between 1941 and 1985. Average age adjusted annual incidence rates peaked at 179/100 000 for men in 195961 and 61/100 000 for women in 1956-58. After peaking a steady downward pattern occurred. From 1941 to 1985 the median age at onset increased for both sexes, from 42 to 56 years for men and from 42 to 55 years for women. The men to women ratio declined from 2.5 to 1.5. By the end of 1987 no Chamorro born after 1949 had developed motor neuron disease. The longest latency or incubation period found was 34 years, a Chamorro born on Guam who went overseas for that time, returned to Guam and developed motor neuron disease. The shortest incubation period was 3 years, found in a Filipino who lived in Guam 36 months before developing the disease. In general the latency period to acquire the disease has increased.

Román analysed the most recent years of meaningful risk for acquiring motor neuron disease based on migrants to Guam. Of six Filipino and 
three US white men who arrived to the islands and developed motor neuron disease, 7 arrived in the late 1940s, one in 1956 and one in 1963. Since 1960 until 1987 no new cases of motor neuron disease occurred in Filipino migrants. Among Chamorro migrants who left Guam between 1934 and 1966 there were 41 cases of motor neuron disease and only 6 cases among later migrants. The conclusion was drawn that 1960-66 seemed to be the most recent years of meaningful risk for acquiring motor neuron disease on Guam.

The critical age of exposure was calculated based on data from Chamorro migrants. High risk exposure only in childhood failed to cause motor neuron disease, whereas high risk exposure during adolescence led to all the known cases. Increase in latency periods and checkline in incidence could indicate a possible dose-response relation.

Notwithstanding familial aggregation the two southern districts of Inarajan and Umatac showed average annual age adjusted incidence rates of motor neuron disease that were significantly higher than the overall rate. Pearson correlation coefficients for reported mean concentrations of cycasin and BMAA in cycad flour were calculated for 9 districts. For men and women a highly significant correlation $(\mathrm{P}=0.00002, \mathrm{r}=0.98)$ between average annual age adjusted incidence rates of motor neuron disease and cycasin could be demonstrated, but not for BMAA.

Family history was available for 303 Chamorros who developed motor neuron disease in Guam between 1956 and 1987. The risk of development of motor neuron disease in susceptible sibships was up to 28 times greater than the general population lifetime risk.

However, the epidemiology of the disease was inconsistent with mendelian inheritance. But it was found conceivable that a susceptibility gene could be present among the Chamorros. The epidemiological evidence provided support for cycas neurotoxicity as an aetiological agent in Guam. No correlation was found of motor neuron disease with calcium, magnesium or aluminium content in soil and water, although a significant correlation did exist with iron concentrations in water samples (Román, 1996).

Zhang et al (1996) discussed the metal intoxication hypothesis, on the basis of the same data used by Román and co-workers. It had been suggested by Yase (1972) and Gajdusek and coworkers during the 1980's that chronic dietary deficiencies of calcium and magnesium could induce secondary hyperparathyoridism leading to increased absorption of toxic metals such as aluminium, and deposition of these metals along with calcium and silicon in neurons. Zhang et al. (1996) argued against this considering the geographic pattern of the disease and the geology of Guam. The incidence of ALS-PD was higher in the some regions of Guam, especially in the south, and the incidence rates of ALS-PD were not significantly correlated with concentrations of calcium, magnesium, aluminium or manganese from water and soil samples taken from those 
districts. The incidence rates were, however, significantly correlated with iron and silicon (men only) in water samples and with cobalt and nickel from soil samples.

Chronic deficiencies of calcium were argued by Zhang and coworkers to adversely affect children and women more than men, but nevertheless men were at higher risk to develop ALS-PD. Moreover, no Chamorro migrant who left Guam as a child was known to have developed the disease.

The familial aggregation of ALS-PD on Guam was not in line with the notion that common sources of water or native plants were responsible for the calcium deficiencies that supposedly gave rise to the disease, unless the metabolic defects that underlie the metal intoxication hypothesis have a genetic basis, according to Zhang et al. (1996).

Whether magnesium depletion was linked to age-related neurodegenerative disease was discussed by Durlach and coworkers (1997). Considering the Western Pacific ALS-PD complex they found that early epidemiological studies suggested a genetic aetiology, but it was not possible to find any simple Mendhelian inheritance patterns, in addition disparate ethnic groups where afflicted. The decline of occurrence in all high incidence foci in the Western Pacific after the second world war, and the absence of transmissible or simple heritable factors focused the search for the cause of the syndrome to environmental factors. Reed et al. (1987) found that consumption of traditional Chamorro food was the only one of 23 tested variables significantly associated with an increased risk for PD. Incrimination of cycad flour containing cycasin and BMAA was an early suggestion. In experimental models neurotoxicity of BMAA varied with cofactors such as pollutant metals, aluminium or manganese. In the three foci of Western Pacific neruodegenerative complex excessive amounts of toxic metals including aluminium and manganese were present in garden soil and drinking water. Metal analysis of CNS tissues from cases showed high levels of Al, Mn and Ca (Gajdusek and Salazar, 1982; Yase, 1972). It was hypothesised that dietary deficiencies of $\mathrm{Ca}$ and $\mathrm{Mg}$ might increase the neurotoxicity of metals. However, Durlach et al. (1997) postulated that nutritional deficiencies of $\mathrm{Ca}$ and $\mathrm{Mg}$ had only been inferred from indirect data, such as low concentration in garden soils and river water, and not from evaluation of dietary intake. Ahlskog et al. (1995) evaluated status of $\mathrm{Ca}, \mathrm{Mg}$ and heavy metals in 12 patients with ALS-PD complex and 12 Chamorro control subjects. Patients had normal values for $\mathrm{Ca}$ in serum and urine. No patients had reduced $\mathrm{Mg}$ and $\mathrm{P}$ values, although a few patients had low urinary $\mathrm{Mg}$ concentrations. Metals (Al, Mn, Pb, Cd, $\mathrm{Cu}, \mathrm{Fe}, \mathrm{Hg}$ and $\mathrm{Zn}$ ) were analysed in serum and urine, and were statistically similar in the patient and control groups except for a slight elevation of $\mathrm{Pb}$ in blood, but not urine, of the patient group. Concentrations of heavy metals in hair and nails were similar in the two groups. In this study there was no evidence to support a major role of $\mathrm{Ca} / \mathrm{Mg}$ deficien- 
cies combined with heavy metals loads in the development of Western Pacific ALS-PD (Durlach et al, 1997).

In the 1980's the average age for onset of ALS in Guam was at about 45 years of age, 20 years younger than in the classical form of ALS and about 5 years earlier than in the familial form as studied in the US and the UK. The lower and upper motor neuron degeneration of the Guam form of ALS is similar to that of the sporadic or classical form of ALS in western countries, with the additional and apparently specific feature of an excess of neurofibrillary tangles and intracytoplasmic (granulovacular) bodies, particularly in the nerve cells of the hippocampus and other subcortical areas. PDC on Guam occurred in the 1980's on the average about 10 years later in life than ALS. It is clinically characterised by a combination of pyramidal signs and symptoms with intellectual deterioration, bradykinesia, rigidity and other parkinsonian features. As in ALS there are widespread subcortical neurofibrillary tangles present in the CNS of patients suffering from PDC. In about one-sixth of those with PDC, clinical and pathological features of ALS where also present. The neurofibrillary tangles are regarded as the common denominator of the Guam CNS disease (Kurland, 1988).

The prevalence of ALS in Guam in the early 1950's was substantially higher than ALS in the white western populations - 50-80/100 000 as comparet to $1-2 / 100000$, respectively. Similar prevalence rates were observed for PDC. The highest prevalence of ALS on Guam was in the village of Umatac, 250/100 000. During the following decades the prevalence of both disorders declined. Estimates for ALS prevalence in Guam for the 1070 -s and 1080 -s range from 30 to less than 5 per 100000 . The prevalence of PDC has also declined, but to a lesser extent. During the same period the age at onset for ALS has increased from 47.6 to 51.9 years, and for PDC from 42.1 to 52.2 years. Whereas the incidence for ALS has declined to a level that is very low, the incidence for PDC is still higher than in the western world. The disease clusters in families and therfore inheritance may contribute to ALS-PDC susceptibility, whereas the change in incidence and onset age suggests that gene-environment interactions are important (Poorkaj et al, 2001).

The year 1958 the population on Guam amounted to 67044 of which 34762 were Chamorro. 1995 the total population was 151724 out of which 65883 were Chamorro. In 2002 the Chamorro population of Guam is a mixture of the original Chamorro people with Filipino, Caucasians and Mexicans. The year 1958 two prospective studies started in Guam consisting of patients and control. The control group were of the same sex as patients, had the same age (within 5 years), came from the same village and had the same marital status. After 25 years, 1983, it was clear that there was a significantly higher risk for ALS and PD among parents, siblings and spouses to patients, but not to relatives of control persons without symptoms. In 1998 a 40-year follow up was carried out. Rela- 
tives, siblings and also wives but not husbands, to patients with ALS had a higher risk for ALS and PD than Chamorro in general. Relatives to the control group did not have an augmented risk for the diseases. Some categories of relatives to patients with PD had a higher risk for both ALS and PD than the population in general, and higher risk for PD than Chamorro in general. The most important finding was that children to patients with PD had an increased risk for ALS and PD, whereas children to patients with ALS did not have an increased risk to acquire the diseases (Plato et al, 2002).

In 1989 the yearly age-adjusted incidens for ALS in Guam was 7/100 000 and for PD 22/100 000. The incidence pattern differed for the two diseases. ALS diminished during the period 1950-1989 but had not disappeared 1989, when the incidence still was higher than in USA and Western Europe. The incidenc for PD was at its peak 1960-1964 for men, and 1970-1979 for women, and the decline in incidence rates were slower than for ALS. During the period 1950-1989 the age of onset of the diseases ALS and PD in Guam increased with about a decade for both men and women. The risk of acquiring the disease is higher for progeny of patients that suffer the disease than for the Chamorro population in general, as is also the case for husbands and wifes to patients. (Waring et al, 2004)

Worms published a review of the epidemiology of motor neuron diseases in 2001. The article reviews 15 epidemiological studies from Europe and North America, 9 of which were published during the 1990s and 6 that were published 1960-1989, and 2 review articles. The sizes of the populations that were studied were between 416000 and 13, 178000 in the articles from the 1990s and between 59000 and 6, 467000 for the articles published 1960-1980. The yearly incidence for motor neuron disease including ALS was 1.89/100 000 in the 1990s and the mortality $1.91 / 100000$. In $1960-1970$ the yearly incidence was $1.3 / 100000$. The increase in incidence seemed to be related to countries in Southern Europe, to women and to persons above 75 years of age. The increase in incidence is connected to a longer length of life and may be connected to the use of better diagnostic tools. However a true increase in incidence cannot be excluded. (Worms, 2001)

Reed et al described several factors to support the hypothesis of environmental factors as the cause of ALS-PD on Guam and Rota. One was the higher than expected occurrence of both diseases in Filipino migrants to Guam, as long as 29 years after their arrival. This implied that exposure during adult life would be sufficient, and indicated that a long latency period would be involved.

The occurrence of ALS and PD among Chamorro migrants to the mainland United States after periods of absence of 1-34 years from Guam suggested that exposure to environmental factors during childhood and adolescence may be sufficient to cause disease 30 years later. 
The absence of the diseases among US military and construction workers and among a small community of Caroline Islanders on Guam suggested that exposure must be longer or more intimate than that of these visitors and migrants, or that genetic susceptibility was involved.

In 1968 Reed and coworkers performed a baseline medical examination and interview with 899 Chamorros in the Mariana Islands. They compiled roosters of persons on Guam and Rota who were aged 20 years or more from a random selection of one of 20 households from each town on Guam and from a total census of Rota. Out of 851 persons in the sample rooster from Guam 628 (74\%) participated in the study, and 337 $(80 \%)$ of the persons in the rooster from Rota The baseline examination included an acculturation questionnaire, and interview for history of medical problems and a physical examination. A traditional food preference score was developed by using reported frequency of eating 18 types of food, half of which were traditional island foods and the other half available western foods. A traditional attitude score was based upon questions regarding what children should be taught about traditional versus modern ways of life. In the 1968 examinations five prevalent cases were diagnosed, one with ALS and one with PD and one with ALS-PD on Guam, and two cases with mixed ALS-PD on Rota. A follow-up study was done 15 years later, in 1983. Of the 894 persons free of ALS and PD in the original study 177 (19.8\%) had died, 382 (42.7\%) were reexamined or interviewed by telephone, $129(14.4 \%)$ had moved of the islands but were known to be alive and reported to be free of ALS and PD, and 206 (23\%) could not be located. Hospital discharge records were examined for 90 of those that could not be found. Death certificates and hospital record were obtained and examined for 166 of the 177 persons that had died.

During the 15-year period new confirmed cases of ALS and PD occurred, including 23 with PD and 5 with ALS, of which on was mixed ALS-PD. All cases of ALS were found on Guam. The small number of cases of ALS and the pattern of decreasing incidence rates were found to be consistent with earlier reports of the disappearance of high rates of ALS in the Mariana Islands. The age-adjusted average annual incidence rates for PD were three times higher than those for ALS.

A comparison was made where the baseline examination characteristics for 23 persons who were to develop PD were compared to 306 who remained free from neurologic disease. The ALS-cases were not included since the number of new cases was so small. Age-adjusted mean values of 23 selected baseline variables were compared including systolic and diastolic blood pressure; serum glucose, cholesterol, uric acid, triglyceride; weight; disease history of diabetes, stroke, asthma, arthritis, kidney disease, hypertension, mental disorder, alcoholism; number of cigarettes per year, number of years lived abroad, number of years at school; number of people with subsistence level occupation; traditional attitude score; 
number of people speaking Chamorro language; traditional food score; and stress symptom score. Of these 23 variables preference for traditional foods was the only significant variable associated with PD incidence. In a table it is shown that among the 23 cases the mean traditional food score was 36 , and among the 306 noncases it was 28 . Bivariate logistic regression with age revealed this the only significant variable associated with PD incidence. In the report it is, however, not stated how the traditional food score was constructed, and in what manner 'scores' were calculated.

The data were in accord with the apparent disappearance of high rates of ALS but did not support a similar decline for PD. Reed et al takes this as an indication that the hypothesis of a common environmental cause for the two diseases is less tenable. Their finding that the PD-cases had a significantly higher preference for traditional food was consistent with a causal hypothesis related to dietary factors. The findings were in agreement with earlier case-control comparisons which indicated that more PD-cases were found in lower subsistence level groups that ate more fish and raw meat and had more exposure to farm animals.

Among the Filipino migrants to Guam nearly all documented cases of ALS and PD lived in the northern and central low-risk areas of Guam, most of them ate commercially available food and had little exposure to cycad nut. The only common reported food was fish. (Reed et al, 1975) These earlier findings did not support the theory that a low-calcium diet and increased intestinal absorption of toxic metals could be causative for the diseases. A neuropathologic study of neurofibrillary degeneration of selected neurons in brains of 69 deceased Chamorro natives of Guam without known clinical evidence of ALS, PD or dementia revealed a high prevalence of neurofibrillary degeneration, $29 \%$ in persons aged 30-39 and $100 \%$ in persons aged $80-89$. The prevalence rates were $70 \%$ both in the southern villages with low calcium levels and the northern and central villages with normal levels. (Reed et al, 1987)

Several investigators have looked for a genetic explanation for the high incidence of ALS/PDC among the Chamorro population of Guam. The reduction of the Chamorro population to a total of less than 2500 in the 1800 s shows a population history that suggests that ALS/PDC may come from a single or small number of genetic founders, particularly in the high-incidence village of Umatac. Poorkaj et al (2001) argued that since neurofibrillary tangles containing tau protein are present in the Guam variant of ALS and PDC, and since mutations in the tau gene cause autosomal dominant frontotemporal dementia, the tau gene could be a candidate gene for ALS/PDC in Guam. The tau gene was evaluated by DNA sequence analysis in subjects with ALS/PDC, by linkage analysis of tau gene polymorphisms and by evaluation of linkage disequilibrium with polymorphic markers flanking and within the tau gene, in a Chamorro population from the village Umatac on Guam, a village with a high incidence. Subjects from Umatac were studied because of the high 
incidence of ALS/PDC in this village. Chamorro controls were healthy, nondemented subjects with no family history of ALS or PDC. Numerous obligate recombinants demonstrate that no specific tau gene allele is required to develop ALS/PDC, though a specific tau gene allele increases susceptibility to the disease. The tau gene may be a modifying gene increasing risk for ALS/PDC in the presence of another, as yet, unidentified gene. The conclusion is based in part on the hypothesis that all cases of ALS/PDC in Guam are the result of the same inherited allele or mutation. (Poorkaj et al, 2001)

Chen et al analysed a genetic risk factor of Alzheimer's disease, apolipoprotein E, which is hypothesised to be linkes to NFT formation, and a genetic risk factor of Parkinson's diseasee, CYP2D6 mutation linked to slower metabolism of exogenous toxins, in Chamorro, Guam individuals with and without PDC. The representation of the G-to-C mutation in exon 9 of the CYP2D6 gene was higher in Chamorro and Filipino than in Caucasian individuals, but this mutant allele had similar high frequencies in both PDC patients and healthy Chamorro individuals. No alleles of these genes associated with $\mathrm{AD}$ or PD was found to be overrepresented among those with PDC. (Chen et al, 1996) 


\section{Annex 2}

Summaries of in vivo toxicological studies

Annex 2 includes summaries of in vivo toxicological studies performed with preparations of cycad, in Annex 2 table 1, and with BMAA, in Annex 2 table 2

\section{Table 1. In vivo studies on cycad preparations}

\begin{tabular}{|c|c|c|c|}
\hline Species & Preparation & \multirow[t]{3}{*}{ Ref. } & \multirow[t]{2}{*}{ Summary of results } \\
\hline $\mathrm{N}: \mathrm{o}$ of animals in control & Route/duration & & \\
\hline $\mathrm{N}: \mathrm{o}$ of animals in dosage groups & Dose & & Comments \\
\hline Rhesus monkey & \multirow{2}{*}{$\begin{array}{l}\text { Two types of cycad flour (washed by Guam natives or } \\
\text { laboratory washed) were incorporated in feed. }\end{array}$} & \multirow{7}{*}{$\begin{array}{l}\text { Dastur and } \\
\text { Pallekar, } 1964\end{array}$} & Pilot study, aiming at studying the toxicity of cycads (not BMAA). \\
\hline 1/control & & & 26-day feeding: Anorexia. Prostration and hemorrhages in arms, gone by day 32. Biopsy of liver after 1 year essentially \\
\hline 1,1 and $1 /$ resp. dosage group & Study duration: 26 days -14 months. & & normal \\
\hline \multirow[t]{4}{*}{ Three treatment groups. } & $\begin{array}{l}\text { - a total of } 15 \mathrm{~g} / 26 \text { days, (last } 18 \text { days containing } \\
\text { approx. } 0.12 \mathrm{~g} \text { cycasin). }\end{array}$ & & $\begin{array}{l}14 \text { months feeding: Hypochromic anemia, no other toxic effects observed. Liver biopsy showed bile duct proliferation } \\
\text { after } 9 \text { months, but no abnormality after } 10.5 \text { months. }\end{array}$ \\
\hline & $\begin{array}{l}\text { - a total of } 254 \mathrm{~g} / 14 \text { months (during last } 4 \text { months } \\
\text { containing approx. } 1.52 \mathrm{~g} \text { cycasin). }\end{array}$ & & \multirow{3}{*}{$\begin{array}{l}\text { 10-months feeding: Leg weakness after } 3.5 \text { months. After } 7 \text { months anorexia. Monkey died after } 10 \text { months, showing } \\
\text { progressive weakness, wasting of muscle in one arm, anemia, elevated SGOT-SGTP. Autopsy showed degeneration of } \\
\text { anterior horn cells, pyramidal neurons of motor cortex. Fibrous scarring of liver. } \\
\text { Pilot study. Important data necessary to evaluate results are missing in the study report. }\end{array}$} \\
\hline & - a total of $95 \mathrm{~g} / 10$ months (during last 2 months con- & & \\
\hline & taining approx. $0.29 \mathrm{~g}$ cycasin). & & \\
\hline
\end{tabular}




\section{Monkey, species not specified.}

3 monkeys/control group

5, 5 and 3 monkeys/resp. dosage

group

Three dosage groups

Male and female Osborn-Mende rats

15 rats/control group

15,15 and 15 rats/resp. dosage group

Three dosage groups.

Male and female Osborne-Mendel and Sprague-Dawley rats.

3,5 or $10 \mathrm{rats} /$ dosage groups, in several different exper

Monkeys (Macaca fascicularis)

2 monkeys/control group

2 monkeys/dosage group

One dosage group.
Washed, unwashed or boiled cycad flour was mixed

into feed.

Lenghth of feeding time not stated.

Dosage was in total: 89 - $1454 \mathrm{~g}$

Washed cycad flour was mixed into feed which was given for 713-717 days.

Yang et al,

$0 ; 1.5 ; 5,0 ; 10 \%$ cycad flour from Cycas circinalis in the feed.

Unwashed cycad flour from Cycas circinalis was mixed into feed and fed for 5 days to 2 weeks.

$0 ; 1 ; 2 ; 3 ; 4 ; 5$ up to $15 \%$ cycad flour in the feed.

Ground dried liver in feed at 2; 5; or 10\%; liver from a heifer fed $2 \%$ unwashed cycad flour.

Unwashed cycad flour from Cycas circinalis was mixed into feed that was given daily for $41-43$ months.

$2 \mathrm{~g}$ in diet

Dastur and 1966
The study aimed at examining the toxicity of different forms of cycad preparations (not BMAA)

Three animals died during the experiment, but the times of deaths were not given.

Washed cycad flour: One monkey showed signs of hemorrhagic disorder and hepatotoxicity, but recovered. One showed slowly progressing hepatotoxicity. One died of infection and malnutrition.

Unwashed cycad flour: Two animals suffered hepatotoxicity and one died in hepatic coma.

Boiled cycad flour: Two animals showed signs of hepatotoxicity.

All groups displayed degenerative changes of liver amounting to cirrhosis.

Important data necessary to evaluate results are missing in the study report.

Object of study: ALS and cancer induction.

11 out of the 45 animals given cycad flour in feed died during the study.

No statistically significant differences between control and dosed animals were found at autopsy or histological examination. However, it is not stated to what extent CNS or PNS was studied.

It is questionable how well the animals were observed and how they were treated. There was a temperature drop in animal quarters that was not observed for so long time that it affected the animals weight. One rat that died was autolysed to such and extent that histological examination could not be performed.

Campbell et al, The object was to investigate the influence of age, strain and diet on weight and weight gain on rats fed unwashed cycad flour. Younger rats were more susceptible to cycad toxicity than older rats.

A heifer that was given $2 \%$ cycad in feed and developed neurological disturbances after 43 days, was slaughtered and the dried, ground liver was fed to weanling rats. Weight gain of treated rats did not differ from control.

Important data necessary for evaluation of results are missing in the study report. Several different experiments are included, but weight is the main parameter that has been studied.

The study is not considered relevant.

arruto et al, $\quad$ All 4 monkeys were fed a low-calcium diet supplemented with high amounts of aluminium and manganese.

No neurological symptoms were observed, however both the dosed and control animals showed motoneuronlesions, possibly as a consequence of metal toxicity.

This is a 'letter to editor', and not a full article. Many details of study are not referred. See Garruto et al 1988 b. 


\section{Monkeys (Macaca fascicularis)}

1 monkey/control group

2, 2 and 2 monkeys/resp. dosage

group

Three treatment groups.

Quackenbush male mice

$\mathrm{N}$ :o of animals not stated in contro

or dosage groups

One control group.

Four dosage groups.

Male CD-1 mice

7 mice/control group

7 mice/dosage group

One dosage group.
Unwashed cycad flour from Cycas circinalis was mixed into feed and fed daily for 4 years.

Control monkey recieved: normal diet.

The three treatment groups were:

a) low Ca diet

b) low Ca diet + high supplementation $\mathrm{Al}$ and $\mathrm{Mn}$

c) low Ca diet + high supplementation $\mathrm{Al}$ and $\mathrm{Mn}+2 \mathrm{~g}$ cycad flour daily

Ground dried nuts of cycad (Lepidozamia per-

offskyana) was mixed into feed and supplied to mice

for 2 days to 6 weeks.

Dose levels in the feed were $0,5,10,15$ or $30 \%$ cycad nut material in diet.

Washed cycad flour from Cycas circinalis was mixed into feed administered for 30 days.

The dose corresponded to $0.5 \mathrm{~g}$ cycad flour/ day

Total average dose was $10.1 \mathrm{~g}$ cycad flour $/$ mouse
Garruto et al,

$1988 \mathrm{~b}$

This refers the genuine article, the same study is referred in letter to editor: Garruto $1988 a$.

All animals remained clinically well.

Neuropathological changes were seen in all animals, but control. Monkeys with low Ca-diet and supplemental Al and Mn had most significant pathology.

The study aims at finding an experimental model for metal neurodegeneration.

It is not possible to assess cycad or BMAA toxicity from the results of this study.

Gobé, 1994 The article refers a reappraisal of archival tissue slides (due to renewed interest in cycad and neurotoxicity). Previously not defined cytological alterations were now defined as apoptosis.

Tissues from control animals appeared normal. Most toxic effects were observed at the two high-dose levels. Live tissue was most affected. On reexamination an increased apoptosis was found in brain tissue of cycad fed mice, but the cell-type that was affected was not identified.

It is difficult to interpret how much bias may be connected with these observations.

Study is not considered relevant

Wilson et al, The cycad flour contained $0.144 \mu \mathrm{g}$ BOAA and $0.003 \mu \mathrm{g}$ BMAA/g, cycasin and MAM was not detectable.

2002 Symptoms of neurological deficiencies were observed in cycad fed mice. Histologically cycad fed mice showed apoposis in regions of the CNS. Tau-positive cells osis in cord. Authors suspected a 'new' toxin to be implicated. 
Table 2. In vivo studies on BMAA.

\begin{tabular}{|c|c|c|c|}
\hline Species & Route/duration & Reference & Result \\
\hline $\mathrm{N}: \mathrm{o}$ of animals in control & Dose & & \\
\hline \multicolumn{3}{|l|}{$\mathrm{N}: \mathrm{O}$ of animal in dosage groups } & Comments \\
\hline Chickens & Single injection & \multirow[t]{3}{*}{ Vega et al, 1968} & \multirow{2}{*}{$\begin{array}{l}\text { Chickens treated with L-isomer showed weakness, convulsions and head retractions that wore off. No symptoms } \\
\text { were observed in the D-isomer-treated group. }\end{array}$} \\
\hline 3 birds/control group & Control: saline & & \\
\hline 3 birds/dosage group & 3-7 $\mu$ moles D- or L-BMAA /g bw & & \multirow[t]{2}{*}{ Important data necessary for evaluation of the results are missing in the study report. } \\
\hline \multicolumn{3}{|l|}{ Two dosage groups. } & \\
\hline Female Wistar rats & Single injection & \multirow[t]{3}{*}{ Vega et al, 1968} & \multirow{2}{*}{$\begin{array}{l}\text { Rats treated with L-isomer showed dragging gait, weakness and convulsions. No symptoms were observed in the } \\
\text { D-isomer-treated group. }\end{array}$} \\
\hline 3 rats/control group & Control: saline & & \\
\hline 3 rats/dosage group & 6-14 $\mu$ moles D- or L-BMAA/g bw & & \multirow[t]{2}{*}{ Important data necessary to evaluate results are missing in the study report. } \\
\hline \multicolumn{3}{|l|}{$\mathrm{N}: \mathrm{o}$ of groups not stated } & \\
\hline Chickens & Single injection i.p. & \multirow[t]{3}{*}{ Polsky et al, 1972} & Toxicity (convulsions) was observed in 25 resp. 11 of the birds in the two high dosage groups. \\
\hline 3 birds/control group & $0 ; 0.20 ; 0.40 ; 0.80 \mathrm{mg}$ DL-BMAA/g bw & & \multirow[t]{2}{*}{ Important data necessary for evaluation of the results are missing in the study report. } \\
\hline $\begin{array}{l}\text { 6, } 28 \text { resp. } 12 \text { birds/resp. dosage } \\
\text { group. Three groups. }\end{array}$ & & & \\
\hline Rats & Single injection i.p. & \multirow[t]{2}{*}{ Polsky et al, 1972} & \multirow{3}{*}{$\begin{array}{l}\text { Toxicity (convulsions) was observed in } 11 \text { resp. } 24 \text { of the animals in the two high dosage groups. } \\
\text { Important data necessary for evaluation of the results are missing in the study report. }\end{array}$} \\
\hline 6rats/control group & $0 ; 0.42 ; 0.84 ; 1.68 \mathrm{mg}$ DL-BMAA/g bw & & \\
\hline 3,12 and $24 /$ resp.dosage group & & & \\
\hline
\end{tabular}




\begin{tabular}{|c|c|c|c|}
\hline $\begin{array}{l}\text { Mice } \\
6 \text { mice/control group } \\
7,7,7 \text { and 4/resp. dosage group } \\
\text { Four dosage groups. }\end{array}$ & $\begin{array}{l}\text { Single injection i.p. } \\
0 ; 0.84 ; 1.68 ; 2.52 ; 3.34 \mathrm{mg} \text { DL-BMAA/g bw }\end{array}$ & Polsky et al, 1972 & $\begin{array}{l}\text { Toxicity (convulsions) was observed in } 1,3 \text { resp. } 4 \text { of the animals in the three high dosage groups. } \\
\text { Important data necessary for evaluation of the results are missing in the study report. }\end{array}$ \\
\hline $\begin{array}{l}\text { Male Sprague-Dawley rats } \\
3 \text { rats/control group } \\
3 \text { rats/dosage group } \\
\text { One dosage group. }\end{array}$ & $\begin{array}{l}\text { Daily injection s.c., } 78 \text { days } \\
0.28 \mathrm{mg} \text { DL-BMAA/g bw/day }\end{array}$ & Polsky et al, 1972 & $\begin{array}{l}\text { No neurologic alterations were observed in treated animals. Weakness and reduced growth rate were observed in } \\
\text { dosed animals, accompanied with a changed amino acid excretion pattern. } \\
\text { Important data necessary for evaluation of the results are missing in the study report. }\end{array}$ \\
\hline $\begin{array}{l}\text { Rats, newborne } \\
\text { N:o of control and dosed animals } \\
\text { have not been stated. }\end{array}$ & $\begin{array}{l}\text { No information on route, treated daily for the } \\
\text { first } 11 \text { days of life. } \\
0.14 \mathrm{mg} \text { DL-BMAA/g bw/day }\end{array}$ & Polsky et al, 1972 & $\begin{array}{l}\text { No neurological symptoms were observed in treated animals. No histological lesions in brain or liver as compared } \\
\text { to control. } \\
\text { Important data necessary for evaluation of the results are missing in the study report. }\end{array}$ \\
\hline $\begin{array}{l}\text { Monkeys (Macaca fascicularis) } \\
5 \text { monkeys/control group } \\
4,4,2(1), 3(1) \text { monkeys/resp. } \\
\text { dosage group }\end{array}$ & $\begin{array}{l}\text { Oral administration but method not given, daily } \\
\text { treatment. Daily treatment for } 5-13 \text { weeks. } \\
100 \mathrm{mg} \text { L-BMAA in aqueous solution/kg } \\
\text { bw/day, } 12 \text { weeks. } \\
250 \mathrm{mg} / \mathrm{kg} \text { bw/day, } 2 \text { weeks then } 125 \mathrm{mg} / \mathrm{kg} \\
\text { bw/day } 5-7 \text { weeks. } \\
200 \mathrm{mg} / \mathrm{kg} \text { bw/day, } 6-10 \text { weeks, } 1 \text { animal } \\
\text { additionally } 250-350 \mathrm{mg} / \mathrm{kg} \text { bw/day } 6 \text { weeks. } \\
300-315 \mathrm{mg} / \mathrm{kg} \text { bw/day, } 2.5-3 \text { weeks, } 1 \text { animal } \\
\text { additionally } 200 \mathrm{mg} / \mathrm{kg} \text { bw/day } 6 \text { weeks. }\end{array}$ & Spencer et al, 1987a & $\begin{array}{l}\text { Treated animals developed corticomotoneuronal dysfunction, parkinsonian features and behavioural anomalies, } \\
\text { along with degenerative changes of motor neurons in cerebral cortex and spinal cord. Muscle weakness and loss of } \\
\text { muscle bulk, forelimbs affected first. } \\
\text { Symptoms temporarily relieved with L-DOPA treatment. }\end{array}$ \\
\hline
\end{tabular}




\begin{tabular}{|c|c|c|}
\hline Female CD-1 mice & Orally by gavage, 11 weeks & Perry et al, 1989 \\
\hline 8, 8 mice/control group. & Control: water & \\
\hline Two control groups. & $500 \mathrm{mg}$ DL-BMAA HCl/kg bw/day for 18 days & \\
\hline 8,12 mice/resp. dosage group & followed by $500 \mathrm{mg} / \mathrm{kg}$ bw every other day 28 & \\
\hline (+ extra animals for analysis of & $\begin{array}{l}\text { days followed by } 1000 \mathrm{mg} / \mathrm{kg} \text { bw every other } \\
\text { day } 30 \text { days. }\end{array}$ & \\
\hline $\begin{array}{l}\text { tissue content of endogenous } \\
\text { substances?) }\end{array}$ & $\begin{array}{l}\text { Injection s.c. of MK- } 801 \text { to one control and one } \\
\text { dosage group, }\end{array}$ & \\
\hline Two dosage groups. & $\begin{array}{l}2 \mathrm{mg} / \text { animal for } 12 \text { days, followed by } 1 \\
\mathrm{mg} / \text { animal for the rest of the study. }\end{array}$ & \\
\hline $\begin{array}{l}\text { Male and female Wistar rats } \\
\text { N:o of animals not given }\end{array}$ & $\begin{array}{l}\text { Single or repeated i.p. injections given at } \\
\text { various intervals during } 2 \text { to }>21 \text { days }\end{array}$ & Seawright et al, 1990 \\
\hline $\mathrm{N}: \mathrm{o}$ of dosage groups not stated. & $\begin{array}{l}\text { L-BMAA: } 6-14 \mu \mathrm{mol} / \mathrm{g} \text { bw (approx. } 0.7-1.6 \\
\mathrm{mg} / \mathrm{g} \text { bw) but in other part of the article the } \\
\text { dose was stated to be } 0.5-2 \mathrm{mg} / \mathrm{g} \mathrm{bw} \text {. }\end{array}$ & \\
\hline & DL-BMAA: $0.35-4 \mathrm{mg} / \mathrm{g}$ bw. & \\
\hline $\begin{array}{l}\text { Male and female Sprague-Dawley } \\
\text { rat pups }\end{array}$ & $\begin{array}{l}\text { Single or once repeated s.c. injections. Dura- } \\
\text { tion of study was } 101 \text { days. }\end{array}$ & Dawson et al, 1998 \\
\hline 24 rats/control group & Control: Phosphate buffered saline. & \\
\hline 12,24 and 12 rats/resp. dosage & 500 mg L-BMAA/kg bw on post-natal day 5. & \\
\hline & $500 \mathrm{mg} / \mathrm{kg}$ bw on post-natal days 2 and 5. & \\
\hline Three dosage groups. & $100 \mathrm{mg} / \mathrm{kg}$ on post-natal days 2 and 5. & \\
\hline $\begin{array}{l}\text { Male CD-1 mice } \\
7 \text { mice/control group }\end{array}$ & $\begin{array}{l}\text { BMAA was mixed into mouse chow pellet that } \\
\text { were fed to mice for } 30 \text { days. }\end{array}$ & $\begin{array}{l}\text { Cruz-Aguado et al, } \\
2006\end{array}$ \\
\hline 7 mice/doagee group & $28 \mathrm{mg} \mathrm{BMAA} / \mathrm{kg}$ bw daily & \\
\hline One dosage group. & & \\
\hline
\end{tabular}

BMAA treatment resulted in no observed behavioural effects. No lesions were observed in brain or spinal cord upon histological examination. Mean contents of dopamine and its metabolites, noradrenaline, serotonin or 5-HIAA were not altered in striata. Mean glycine levels were significantly lower in cortices of mice fed BMAA than in controls.

Study appears to be well conducted and referred, although it is difficult to interpret the total number of animals in the study.

Both the racemate and L-form produced acute signs of cerebellar dysfunction and degeneration of cerebellar stellate, basket, Purkinje and Golgi cells, but not granule cells.

Study very poorly referred. It is impossible to interprete dosages, dosage regims and number of animals used.

Treatment related deaths were 0/24 (control), 1/12 (500 mg/kg once), 7/24 (500 mg/kg twice), 0/12 (100 mg/kg twice).

BMAA given neonatally produced changes in motor function and spinal cord neurochemistry, The observed permanent changes in adult neurochemistry may be related to reorganisational effects induced by toxin-mediated neuroplasticity in developing neurons. The pattern of neurochemical changes was not consistent with the changes reported in postmortem tissue from ALS patients.

Appears to be a well performed study.

No differences were observed between BMAA-fed animals and control as measured in a battery of behavioural test (rotarod, leg extension, stride length, open field, wire hang, radial arm water maze) or by neuropathological analysis of brains and spinal cords.

Appears to be a well performed and well reported study. 